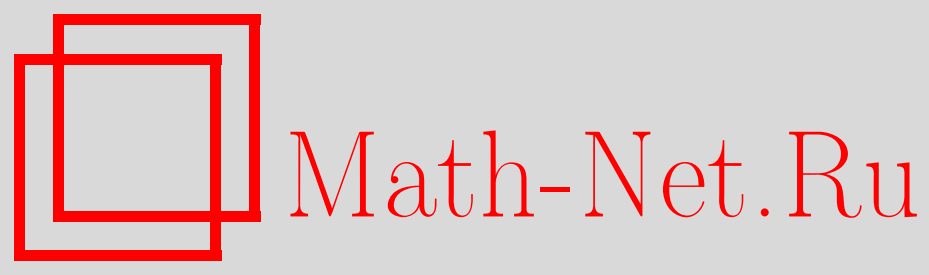

А. И. Аптекарев, В. И. Буслаев, А. МартинесФинкельштейн, С. П. Суетин, Аппроксимации Паде, непрерывные дроби и ортогональные многочлены, УМН, 2011, том 66, выпуск 6, 37-122

DOI: https://doi.org/10.4213/rm9448

Использование Общероссийского математического портала Math-Net.Ru подразумевает, что вы прочитали и согласны с пользовательским соглашением http://www . mathnet.ru/rus/agreement

Параметры загрузки:

IP : 3.85 .183 .62

26 апреля 2023 г., 12:32:36

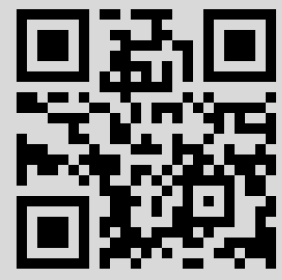




\title{
Аппроксимации Паде, непрерывные дроби и ортогональные многочлены
}

\author{
А. И. Аптекарев, В. И. Буслаев, \\ А. Мартинес-Финкельштейн, С. П. Суетин
}

В работе дается обзор результатов, составляющих основу современной теории сходимости аппроксимаций Паде.

Библиография: 204 названия.

Ключевые слова: рациональные приближения, непрерывные дроби, ортогональные многочлены, аппроксимации Паде, аппроксимации Эрмита-Паде, эффективное аналитическое продолжение, сходимость по емкости, асимптотика полюсов, локализация особенностей, прямые задачи, обратные задачи.

\section{СОДЕРЖАНИЕ}

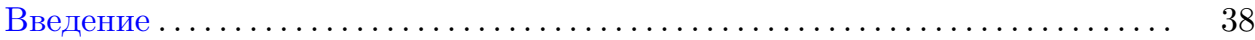

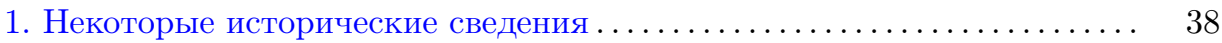

2. Основные понятия и сводка основных результатов .............. 44

Глава 1. Асимптотические свойства ортогональных многочленов и сходимость аппроксимаций Паде ......................... 52

1. Сходимость аппроксимаций Паде для функций марковского типа ... 52

2. Сходимость аппроксимаций Паде для гиперэллиптических функций 58

3. Теорема Шталя . . . . . . . . . . . . . . . . . . . . . . . . . . 62

4. Равновесная мера и распределение нулей экстремальных многочленов 65

5. Одна обратная теорема о диагональных аппроксимациях Паде..... 74

6. О некоторых гипотезах в теории аппроксимаций Паде ........... 77

Глава 2. Скорость рациональной аппроксимации аналитических функций 86

1. Основные понятия и постановка задачи ................. 86

2. Формулировки теорем о скорости рациональной аппроксимации.... 89

Работа первого автора выполнена при поддержке РФФИ (гранты № 11-01-00245, 11-01-12045-офи-м), программы № 1 Отделения математических наук РАН, программы "Саtedras de Exelencia” (Университет им. Карлоса III, Мадрид, Испания, и банк Santander); работа второго и четвертого автора выполнена при поддержке РФФИ (грант № 11-01-00330) и программы "Ведущие научные школы" (грант НШ-8033.2010.1); работа третьего автора выполнена при поддержке Junta de Andalucía (гранты FQM-229, P09-FQM-4643) и Министерства науки и инноваций Испании (код проекта МТМ2008-06689-C02-01).

(С) А. И. АПтекарев, В. И. Буслаев, А. МартинеС-Финкельштейн, С. П. Суетин, 2011 
Глава 3. Непрерывные дроби и рекуррентные соотношения.............

1. Рекуррентные соотношения, теоремы Пуанкаре и Перрона и их приложения к теории сходимости непрерывных дробей ............ 94

2. Гипотеза Лейтона......................................... 101

3. Сходимость непрерывной дроби Роджерса-Рамануджана ........... 102

4. Некоторые приложения уточненной теоремы Пуанкаре ............ 105

Список литературы .................................... 109

\section{Введение}

\section{1. Некоторые исторические сведения}

1.1. Теория аппроксимаций Паде - локально наилучиих рационалъных аппроксимаций степенного ряда - составляет самостоятельное направление в комплексном анализе и теории приближений. В XVIII-XIX вв. это направление развивалось в основном в рамках классической теории непрерывных дробей. Интерес к аппроксимациям Паде и более общим конструкциям рациональных аппроксимаций аналитических функций резко возрос во второй половине прошлого века. Благодаря развитию вычислительной техники такие аппроксимации нашли многочисленные применения в различных задачах математической физики, механики и прикладной математики. В свою очередь, анализ возникающих при этом математических проблем привел к новым задачам и результатам в комплексном анализе, теории потенциала, теории ортогональных многочленов. Подчеркнем, что речь идет о приближениях рациональными функциями со свободными полюсами, а сами рассматриваемые аппроксимации - непрерывные дроби, аппроксимации Паде и их различные обобщения - имеют нелинейный характер. Оптимальный выбор коэффициентов как числителей, так и знаменателей аппроксимаций Паде позволяет с их помощью локализовать особенности функции, заданной своим разложением в степенной ряд, и осуществлять ее эфбективное аналитическое продолжение за пределы круга сходимости исходного ряда.

Понятие аппроксимации Паде сформировалось в конце XIX в., прежде всего, в работах Г. Фробениуса [1] и А. Паде [2]. Фундаментальные результаты о диагональных аппроксимациях Паде были получены в рамках теории непрерывных дробей П. Л. Чебышёвым [3], А. А. Марковым [4] и Т. Стилтьесом [5] (см. также [6]-[13]). Ими были открыты связи аппроксимаций Паде с общими ортогональными многочленами, квадратурными формулами, проблемой моментов, спектральной теорией операторов и другими классическими задачами анализа. Отметим, что впервые общую (формальную) задачу интерполяции значений функции, заданных в $n+m+1$ различных точках, посредством рациональной функции степени не выше $(n, m)$ рассмотрел О. Коши [14]; К. Якоби [15] распространил результаты Коши на случай кратной интерполяции; $(n+m+1)$-кратная интерполяция в одной точке приводит к аппроксимации Паде типа $(n, m)$ (подробнее см. ниже п. 2.1). Конструкция, подобная непрерывной дроби, но соответствующая одновременной аппроксимации нескольких 
функций, была разработана Ш. Эрмитом [16] и легла в основу его доказательства трансцендентности числа $e$. Начало изучению строк таблицы Паде положили работы Ж. Адамара [17] о радиусах кругов мероморфности заданного степенного ряда и Р. Монтессу [18] о равномерной сходимости строк в кругах мероморфности (т. е. за пределами круга сходимости исходного степенного ряда), см. п. 2.2 .

C начала XX столетия аппроксимации Паде становятся самостоятельным объектом анализа, их изучение составляет важный раздел в теории рациональной аппроксимации аналитических функций (см. [8], [11] и [12]). Использование аппроксимаций Паде позволяет, опираясь на локально заданный степенной ряд, изучать глобальные свойства определяемой рядом аналитической функции: расположение и характер ее особых точек, максимальные области однозначной аналитической продолжаемости и т. п. В теории сходимости аппроксимаций Паде основной интерес представляют результаты двух типов - прямые и обратные. В прямых теоремах на основе заранее известной информации о свойствах аналитического продолжения заданной степенным рядом функции делаются те или иные выводы о сходимости соответствующих аппроксимаций, асимптотическом поведении их полюсов и т. п. При общих предположениях об аналитических свойствах заданной функции аппроксимации Паде могут иметь “ложные" полюсы в области голоморфности этой функции (см. [8; ч. I, гл. 2, $\S 2.2]$ ); поэтому в соответствующих теоремах общего характера речь идет о сходимости вне "малых" исключительных множеств. Наиболее естественные результаты о сходимости аппроксимаций Паде привлекают понятие логарифмической емкости. В обратных теоремах исходные данные связаны с самими аппроксимациями Паде, как правило - с предельным поведением их полюсов. На основе этих данных делаются те или иные выводы об аналитических свойствах приближаемой функции.

1.2. Основы современной теории аппроксимаций Паде были заложены в 70-80-х годах прошлого века в работах А. А. Гончара [19]-[31] и Дж. Наттолла [32]-[40]. Полученные ими в этот период результаты и поставленные задачи определили главные направления исследований в области теории конструктивных рациональных аппроксимаций на многие годы вперед.

Прежде всего А. А. Гончаром была решена естественная задача: обобщение классической теоремы Маркова (см. п. 2.3) для диагональных аппроксимаций Паде на комплексный случай. В 1975 г. в работе [22] (см. также п. 1.1 главы 1) им была доказана сходимость аппроксимаций Паде для функций вида $f=\widehat{\sigma}+r$, где $\widehat{\sigma}$ - марковская функция, носитель меры $\sigma$ - отрезок $[a, b]$ вещественной прямой, $\sigma^{\prime}>0$ почти всюду на $[a, b]$, а $r-$ комплексная ${ }^{1}$ рациональная функция, не имеющая на $[a, b]$ ни нулей, ни полюсов. Конструкция аппроксимаций Паде существенно нелинейна, поэтому исследование сходимости этих аппроксимаций для возмущенной марковской функции - сложная и содержательная задача. После теоремы Маркова этот результат стал первым утверждением общего характера о сходимости диагональных аппроксимаций Паде в классах функций. Полученный А. А. Гончаром результат носит принципиальный характер:

\footnotetext{
${ }^{1}$ Результат А.А. Гончара имеет более общий характер: мера $\sigma$ тоже может быть комплексной.
} 
из него вытекает, что диагональные аппроксимации Паде, которые строятся непосредственно по лорановским коэффициентам ряда, в классе функций вида $f=\widehat{\sigma}+r$ при указанных условиях эффективно решают задачу мероморфного продолжения в область $D_{f}=\overline{\mathbb{C}} \backslash[a, b]$ функции, заданной своим разложением в ряд. Это замечательное свойство аппроксимаций Паде основано на том, что все их конечные полюсы свободны и определяются только условием максимального касания в бесконечно удаленной точке (см. п. 2.1). Впоследствии этот результат А. А. Гончара стал широко известен и получил разнообразные интерпретации и дальнейшие обобщения, в частности - в спектральной теории оператора Якоби (см. [41], [42]). Аналогичная теорема для случая нескольких отрезков была доказана в работе [43] в 1977 г.

В второй половине 1970-х годов Дж. Наттолл в серии работ [35], [36] (см. также [40] и ниже п. 2.1 главы 1) получил другой комплексный аналог теоремы Маркова. Вместо нескольких отрезков на вещественной прямой он рассмотрел ситуацию, когда носитель меры состоит из нескольких попарно непересекающихся дуг, расположенных в комплексной плоскости. Эти дуги возникают как линии уровня некоторого абелева интеграла третьего рода с чисто мнимыми периодами, заданного на двулистной гиперэллиптической римановой поверхности. Сама мера предполагается достаточно гладкой, а именно такой, чтобы можно было применить классический аппроксимационный метод Бернштейна-Сегё (см. [44], [45]).

1.3. В начале 1980-х годов в связи с изучением сходимости аппроксимаций Эрмита-Паде А. А. Гончаром и Е. А. Рахмановым был разработан принципиально новый метод решения классических задач теории рациональных аппроксимаций, основанный на применении векторных теоретико-потенциальных задач равновесия с внешним полем (см. [26], [46]-[49], а также [12] и ниже раздел 4 главы 1). Точнее, ими было замечено, что решение ряда задач теории приближений может быть сведено к задаче о равновесном распределении заряда на "проводнике" при наличии тех или иных "внешних полей" (см. ниже теорему 6). В результате возникло знаменитое теперь "соотношение равновесия" для логарифмического потенциала $V^{\lambda}(x)$ на вещественной прямой $\mathbb{R}$ во внешнем поле потенциала $f(x)$, которое изначально было записано (см. [47], [49]) в следующей форме Гончара-Рахманова:

$$
\begin{aligned}
\left(V^{\lambda}+f\right)(x) & =w, \quad x \in S_{\lambda}, \\
& \geqslant w, \quad x \in F \backslash S_{\lambda},
\end{aligned}
$$

где $S_{\lambda}$ - носитель равновесной меры $\lambda=\lambda(f), w=w(f)$ - соответствующая постоянная равновесия (подробнее см. раздел 4 главы 1). Это соотношение ${ }^{2}$

\footnotetext{
${ }^{2}$ Независимо и позже работы [47] соотношения (1) были получены в работе [50]; в широко известной монографии [51] отсутствуют ссылки на оригинальные работы [52], [47]. Отметим, что впервые соотношения вида (1) возникли еще в 1981 г. в работе [26; формулы (27) и (28)], посвященной аппроксимациям Эрмита-Паде для случая Анжелеско [53], [54] в связи с явлением "сталкивания" носителя равновесной меры (см. также [52], [46], [50], [55]-[57] и раздел 4 главы 1).
} 
однозначно определяет меру $\lambda$ (в классе единичных мер на вещественной прямой), а тем самым, и константу $w$. Соотношение (1) решает задачу слабой (логарифмической) асимптотики для многочленов, ортогональных ${ }^{3}$ относительно переменного (зависящего от номера многочлена) веса (см. раздел 4 главы 1). А именно, асимптотическая формула для ортонормированных многочленов $P_{n}^{*}(z)$ в терминах равновесной меры $\lambda$ имеет следующий вид:

$$
\lim _{n \rightarrow \infty}\left|P_{n}^{*}(z)\right|^{1 / n}=e^{w-V^{\lambda}(z)}, \quad z \in \mathbb{C} \backslash F .
$$

Впоследствии оказалось, что соотношения (1) и (2), полученные впервые А. А. Гончаром и Е. А. Рахмановым в 1984 г., имеют самое непосредственное отношение к задачам об асимптотических свойствах случайных матриц. Соотношение равновесия (1) носит чрезвычайно общий характер и является исходным пунктом для дальнейших исследований, связанных со случайными матрицами (см. прежде всего [58; гл. 6, §6], а также [59]-[62] и имеющуюся там литературу). Оно неизменно присутствует практически во всех работах, посвященных этой тематике.

Необходимо отметить, что основной неизвестный параметр задачи (1) - носитель равновесной меры $S_{\lambda}$. Даже для достаточно простых функций $f$ нахождение равновесной меры $\lambda=\lambda(f)$ и экстремальной константы $w=w(f)$ затрудняется тем, что этот носитель заранее не известен. В приложениях, связанных с изучением свойств случайных матриц, наиболее часто встречается ситуация, когда внешний потенциал $f(x)=Q(x)$ - полином четной степени с положительным старшим коэффициентом. В этом случае носитель равновесной меры $S_{\lambda}$ состоит из конечного числа отрезков, а сама равновесная мера абсолютно непрерывна относительно меры Лебега. Однако задача нахождения в явном виде концевых точек этих отрезков по заданному полиному $Q(x)$ в общем случае до сих пор не решена, известно только несколько частных случаев, в которых удается найти явный ответ (см. раздел 4 главы 1).

1.4. Принципиальным моментом для всего дальнейшего развития теории аппроксимаций Паде в общих классах аналитических функций оказалось привлечение А.А. Гончаром и Дж. Наттоллом для описания сходимости таких аппроксимаций понятия логарифмической емкости. Именно в терминах сходимости по емкости Г. Шталем в 1985-1986 гг. в серии работ [63]-[67] (см. также [68]-[70] и раздел 3 главы 1) был установлен один из основных результатов 4 современной теории аппроксимаций Паде: сходимость диагональных аппроксимаций Паде в классе аналитических функций с конечным числом особых точек многозначного характера. Точнее, пусть $\Sigma=\left\{a_{1}, \ldots, a_{p}\right\} \subset \mathbb{C}$ - конечное множество, $\mathscr{A}(\overline{\mathbb{C}} \backslash \Sigma)$ - класс всех функций, голоморфных в точке $z=\infty$ и допускающих аналитическое продолжение вдоль любого пути, лежащего в $\overline{\mathbb{C}} \backslash \Sigma$;

\footnotetext{
${ }^{3}$ Подчеркнем, что результаты работы [47] носят характер гораздо более общий, чем указано здесь, и относятся не только к ортогональным, но и к более общим экстремалъным многочленам.

${ }^{4}$ Отметим, что замечательные результаты Г. Шталя [63]-[67] носят характер гораздо более общий, чем указано здесь, и относятся к многозначным аналитическим функциям, множество особенностей которых составляет множество нулевой (логарифмической) емкости; кроме того, им были получены аналогичные результаты о сходимости наилучших равномерных рациональных аппроксимаций для многозначных аналитических функций.
} 
при этом предполагается, что соответствующая полная аналитическая функция многозначна. Для функции $f \in \mathscr{A}(\overline{\mathbb{C}} \backslash \Sigma)$ через $\mathscr{G}_{f}$ обозначим класс всех областей $G \subset \overline{\mathbb{C}}$, содержащих бесконечно удаленную точку и таких, что функция $f$ продолжается в область $G$ как голоморфная (т. е. однозначная аналитическая) функция; пусть $\mathscr{K}_{f}=\left\{K: K=\mathbb{C} \backslash G, G \in \mathscr{G}_{f}\right\}$. Области $G \in \mathscr{G}_{f}$ и компакты $K \in \mathscr{K}_{f}$ будем называть допустимымми для функции $f$. Пусть $[n / n]_{f}$ - последовательность диагональных аппроксимаций Паде функции $f$ (в точке $z=\infty$ ). Справедлива следующая теорема.

ТЕОРемА ШТАЛЯ. Пусть $f \in \mathscr{A}(\overline{\mathbb{C}} \backslash \Sigma)$. Тогда в классе $\mathscr{G}_{f}$ существует единственная область $D, D=D_{f} \in \mathscr{G}_{f}$, максимальная по включению и такая, чmo $[n / n]_{f} \stackrel{\text { cap }}{\longrightarrow} f$ npu $n \rightarrow \infty$ в $D$.

Здесь " $\stackrel{\text { сар }}{\longrightarrow}$ " означает сходимость по емкости на компактных подмножествах области $D$. Дополнение $F=\overline{\mathbb{C}} \backslash D$ к $D$ оказывается минимальным в том смысле, что компакт $F \in \mathscr{K}_{f}$ имеет наименьшую емкость в классе всех допустимых компактов $\mathscr{K}_{f}$. При доказательстве этой теоремы Шталь открыл следующий замечательный факт. Оказалось, что экстремальный компакт $F$ обладает определенным свойством "симметрии", или $S$-свойством, а именно: компакт $F$ не разбивает плоскость, состоит из конечного числа аналитических дуг и для логарифмического потенциала $V^{\lambda}(z)$ равновесной меры $\lambda=\lambda_{F}$ компакта $F$ выполняется соотношение:

$$
\frac{\partial V^{\lambda}}{\partial n_{+}}(z)=\frac{\partial V^{\lambda}}{\partial n_{-}}(z), \quad z \in F^{0}
$$

здесь $F^{0}$ - совокупность открытых аналитических дуг, составляющих $F$, и $\partial n_{ \pm}-$производные по нормали с противоположных сторон $F^{0}$ (точные определения см. в главе 2). Указанное свойство симметрии однозначно выделяет компакт $F$ в классе допустимых компактов $\mathscr{K}_{f}$; это свойство играет исключительную роль при исследовании асимптотических свойств знаменателей диагональных аппроксимаций Паде - неэрмитово ортогональных многочленов, а также при исследовании сходимости диагональных аппроксимаций Паде для многозначных аналитических функций.

1.5. Следующий принципиальный шаг в этом направлении был сделан в 1986 г. А. А. Гончаром и Е. А. Рахмановым в работе [49] (см. также [71]), где понятие симметричного компакта являлось исходным при постановке некоторой достаточно общей задачи о наилучшей рациональной аппроксимации аналитических функций (см. главу 2). Точнее, в [49] было впервые введено понятие компакта, обладающего $S$-свойством во внешнем поле $f$, как такого допустимого компакта $F$, который не разбивает плоскость, состоит из конечного числа аналитических дуг и удовлетворяет следующему условию симметрии:

$$
\frac{\partial\left(V^{\lambda}+f\right)}{\partial n_{+}}(z)=\frac{\partial\left(V^{\lambda}+f\right)}{\partial n_{-}}(z), \quad z \in \Gamma^{0},
$$

где $\Gamma=S_{\lambda} \subset F, \lambda=\lambda_{F}-$ соответствующая равновесная мера:

$$
\begin{aligned}
\left(V^{\lambda}+f\right)(x) & =w, \quad x \in \Gamma, \\
& \geqslant w, \quad x \in F \backslash \Gamma,
\end{aligned}
$$


$w$ - постоянная равновесия (точные формулировки см. в разделе 4 главы 1). В предположении существования такого компакта для некоторой теоретико-потенциальной задачи равновесия с гармоническим внешним потенциалом $f$ в [49] была доказана общая теорема о существовании слабой асимптотики для соответствующих задаче ортогональных многочленов с переменным весом и о скорости сходимости наилучших рациональных аппроксимаций. Затем на основе этой общей теоремы в [49] была решена широко известная “задача об 1/9" (так называемая "1/9-conjecture", см. прежде всего [72; гл. 9], а также [73]-[76]). А именно, для этой конкретной задачи в [49] был построен компакт, обладающий $S$-свойством (4) во внешнем поле $f(z)=\operatorname{Re}(z / 2)$, найден носитель соответствующей равновесной меры $\lambda$ (оказалось, что это одна дуга, соединяющая некоторую точку $a$ с сопряженной точкой $\bar{a}$ ) и вычислена соответствующая постоянная равновесия $w$. После этого было доказано, что для величины $\rho_{n}$ равномерного на вещественной полуоси $[0,+\infty)$ уклонения функции $e^{-x}$ от класса рациональных дробей порядка не выше $n$ cyществует nредел $\lim _{n \rightarrow \infty} \rho_{n}^{1 / n}=e^{-2 w}$, а величина $v=e^{-2 w}$ является единственным положительным корнем ${ }^{5}$ уравнения

$$
\sum_{k=1}^{\infty} a_{k} v^{k}=\frac{1}{8}, \quad \text { где } a_{k}=\left|\sum_{d \mid k}(-1)^{d} d\right| .
$$

Этот результат был доложен А. А. Гончаром в 1986 г. на Математическом конгрессе в Беркли (США) и получил широкое международное признание.

Подчеркнем следующее. Первая задача равновесия в поле (1), впервые рассмотренная в [47], изначально ставится на вещественном промежутке $F \subset \mathbb{R}$. Таким образом, в этом случае условие симметрии (4) уже оказывается заранее выполненным. Основная трудность - найти носитель равновесной меры $S_{\lambda} \subset F$, например, концевые точки неизвестных отрезков для внешнего потенциала вида $f(x)=Q(x)$, где $Q$ - полином. В задаче, рассмотренной Шталем, внешнее поле вообще отсутствует. Тем самым, в этом случае носитель равновесной меры - это весь компакт F. Вторая задача равновесия в поле (4), (5), естественным образом впервые возникшая в работе [49], оказалась сложнее: она включает в себя как непосредственно соотношение равновесия (5), так и свойство симметрии (4) заранее неизвестной кривой. В случае полиномиального внешнего потенциала $f(z)=\operatorname{Re} Q(z)$ из совокупности соотношений (4) и (5) в силу условий Коши-Римана вытекает, что сумма $\mathscr{V}_{\lambda}^{\prime}(z)+Q^{\prime}(z)$ непрерывно продолжается на обе стороны любой дуги носителя равновесной меры $S_{\lambda}$ и ее значения на разных сторонах этих дуг отличаются только знаком; здесь $\mathscr{V}_{\lambda}(z)=-\int \log (z-t) d \lambda(t)$ - комплексный потенциал меры $\lambda$. Тем самым квадрат указанной суммы продолжается через эти дуги как голоморфная функция. В работе [49] используется именно условие продолжаемости такой суммы для $Q(z)=z / 2$ и в результате предъявляется компакт, обладающий $S$-свойством. Решить такую задачу в явном виде удается пока (так же как и в [49]) только

\footnotetext{
${ }^{5}$ Величина $v$ возникла как корень уравнения (6) еще в 1886 г. (ровно за сто лет до работы [49]) в работе Альфана [77] в связи с его исследованиями по вариациям тэта-функций.
} 
в случае, когда носитель равновесной меры оказывается состоящим из одной дуги (так называемый "one-cut case"; см., например, [78]-[82]).

В 2001 г. А. И. Аптекарев [83] доказал еще одну общую теорему (см. ниже главу 2, теорему 12), описывающую точные константы приближения рациональными дробями порядка $n$ аналитических функций из некоторого достаточно общего класса. Основное утверждение ${ }^{6}$ этой теоремы Аптекарева состоит в следующей формуле сильной асимптотики для величины $\rho_{n}$ равномерного на вещественной полуоси $[0,+\infty)$ уклонения функции $e^{-x}$ от класса рациональных дробей порядка не выше $n$ :

$$
\rho_{n}=2 v^{n+1 / 2}(1+o(1)), \quad n \rightarrow \infty,
$$

где $v$ - решение уравнения (6). Формула (7) доказывает справедливость гипотезы А. Магнуса [84] (см. также [85]-[87]) о точной константе рационального приближения функции $e^{-x}$ на полуоси $[0,+\infty)$ (ср. раздел 6 в главе 1 , гипотеза 6.10).

\section{2. Основные понятия и сводка основных результатов}

2.1. Аппроксимации Паде - это локально наилучшие рациональные аппроксимации степенного ряда; в более широком смысле - конструктивные рациональные аппроксимации со свободными полюсами.

Пусть

$$
f=\sum_{k=0}^{\infty} c_{k} z^{k}
$$

- (формальный) степенной ряд, $n, m$ - произвольные целые неотрицательные числа. Аппроксимацией Паде типа $(n, m)$ ряда $f$ называется рациональная функция $P_{n, m} / Q_{n, m}$, где полиномы $P_{n, m}, \operatorname{deg} P_{n, m} \leqslant n$, и $Q_{n, m}, \operatorname{deg} Q_{n, m} \leqslant m$, $Q_{n, m} \not \equiv 0$, определяются (не единственным образом) из соотношения

$$
\left(Q_{n, m} f-P_{n, m}\right)(z)=O\left(z^{n+m+1}\right), \quad z \rightarrow 0
$$

(в правой части (9) стоит ряд по степеням $z$, начинающийся с $z^{n+m+1}$ ). Из соотношения (9) рациональная функция $[n / m]_{f}=P_{n, m} / Q_{n, m}$ определяется единственным образом. В соответствии с (9) нахождение полинома $Q_{n, m}=q_{0}+$ $\cdots+q_{m} z^{m}, q_{j}=q_{j}(n, m)$, сводится к решению системы $m$ линейных однородных уравнений

$$
\begin{aligned}
& c_{n-m+1} q_{m}+\cdots+c_{n+1} q_{0}=0, \\
& \ldots \ldots \ldots \ldots \ldots \ldots \ldots \ldots \ldots \ldots \ldots \\
& c_{n} q_{m}+\cdots+c_{n+m} q_{0}=0
\end{aligned}
$$

c $(m+1)$ неизвестными. После вычисления знаменателя $Q_{n, m}$ числитель $P_{n, m}$ определяется равенством $P_{n, m}=\sum_{k=0}^{m} c_{k}\left(Q_{n, m} f\right) z^{k}$, где $c_{k}(\cdot)-k$-й коэффициент ряда Тейлора, стоящего в скобках.

\footnotetext{
${ }^{6}$ Результат А. И. Аптекарева носит гораздо более общий по сравнению с (7) характер и относится не только к конкретной функции $e^{-x}$, но и к целому классу функций определенного вида.
} 
Если отличен от нуля определитель Адамара

$$
H_{n, m}=\left|\begin{array}{cccc}
c_{n-m+1} & c_{n-m+2} & \ldots & c_{n} \\
\ldots \ldots \ldots \ldots \ldots \ldots \ldots \ldots \ldots \ldots \\
c_{n} & c_{n+1} & \ldots & c_{n+m-1}
\end{array}\right|
$$

(полагаем $c_{k}=0$ при $k<0$ ), то для знаменателя $Q_{n, m}$ имеет место явная формула

$$
Q_{n, m}(z)=\frac{1}{H_{n, m}}\left|\begin{array}{cccc}
c_{n-m+1} & c_{n-m+2} & \ldots & c_{n+1} \\
\ldots \ldots \ldots & \ldots \ldots \ldots \ldots \ldots \ldots \ldots \\
c_{n} & c_{n+1} & \ldots & c_{n+m} \\
z^{m} & z^{m-1} & \ldots & 1
\end{array}\right|
$$

с нормировкой $Q_{n, m}(0)=1$; аналогичная формула имеет место и для $P_{n, m}$. В этом случае из (9) вытекает, что

$$
[n / m]_{f}(z)=c_{0}+c_{1} z+\cdots+c_{n+m} z^{n+m}+O\left(z^{n+m+1}\right) .
$$

Последнее соотношение иногда принимают (см. [8]) за определение аппроксимаций Паде; при таком подходе аппроксимации Паде для некоторых $n, m$ могут не существовать.

Таблица $\left\{[n / m]_{f}, n, m=0,1, \ldots\right\}$ называется таблицей Паде ряда $f$. Последовательность $\left\{[n / m]_{f}, n=0,1, \ldots\right\}$ ( $m$ - фиксировано) называется $m$-й строкой таблицы Паде; нулевая строка состоит из частичных сумм ряда (8). Последовательности вида $\left\{[n+j / n]_{f}, n=0,1, \ldots\right\}$ и $\left\{[n / n+j]_{f}, n=0,1, \ldots\right\}$, где $j \geqslant 0$ - фиксированное целое число, называются диагональными последовательностями таблицы Паде; последовательность $\left\{[n / n]_{f}, n=0,1, \ldots\right\}$, называется главной диагональной последовательностью или главной диагональю таблицы Паде.

Пусть $\mathscr{R}_{n, m}$ - класс всех рациональных функций вида $p / q$, где $\operatorname{deg} p \leqslant n$, $\operatorname{deg} q \leqslant m, q \neq \equiv 0$. Если $[n / m]_{f}=P_{n, m}^{*} / Q_{n, m}^{*}$, где НОД $\left(P_{n, m}^{*}, Q_{n, m}^{*}\right)=1$, то из (9) вытекает, что

$$
\left(f-[n / m]_{f}\right)(z)=O\left(z^{n+m+1-\lambda_{n, m}}\right),
$$

где $\lambda_{n, m}=\min \left(n-\operatorname{deg} P_{n, m}^{*}, m-\operatorname{deg} Q_{n, m}^{*}\right)-$ дефекm рациональной функции в классе $\mathscr{R}_{n, m}$. Тем самым, аппроксимация Паде типа $(n, m)$ ряда $f$ доставляет максимально возможный порядок касания к этому ряду (в точке $z=0$ ) в классе $\mathscr{R}_{n, m}$, иными словами, является локально наилучшей рациональной аппроксимачией заданного степенного ряда в классе $\mathscr{R}_{n, m}$.

Индекс $(n, m)$ называется нормалъным в таблице Паде, если $\operatorname{deg} P_{n, m}^{*}=n$, $\operatorname{deg} Q_{n, m}^{*}=m$. Критерий нормальности индекса выражается условием: $H_{n, m}$. $H_{n+1, m} \cdot H_{n, m+1} \neq 0$. Для такого индекса при условии, что $f \notin \mathscr{R}_{n, m}$, выполняется соотношение

$$
\left(f-[n / m]_{f}\right)(z)=A_{n, m} z^{n+m+1+\ell_{n, m}}+\cdots,
$$


где $A_{n, m} \neq 0, \ell_{n, m} \geqslant 0$. Нормальными индексами ${ }^{7}$ таблица Паде произвольного ряда $f$ разбивается на квадратные блоки размера $\ell_{n, m} \times \ell_{n, m}:\left[n^{\prime} / m^{\prime}\right]_{f}=$ $[n / m]_{f}$ для всех $n \leqslant n^{\prime} \leqslant n+\ell_{n, m}, m \leqslant m^{\prime} \leqslant m+\ell_{n, m}$ (при $f \in \mathscr{R}_{n, m}$ полагают $\left.\ell_{n, m}=\infty\right)$.

Критерий Кронекера рациональности $f$ состоит в следующем:

$$
f \in \mathscr{R}_{n, m} \quad \Leftrightarrow \quad H_{n+j, m+1}=0, \quad j=1,2, \ldots
$$

Индекс $n$ называется нормальным для главной диагонали, если порядок рациональной функции $[n / n]_{f}$ равен $n$; для произвольного $n$ справедливо равенство $[n / n]_{f}=\left[n_{k} / n_{k}\right]_{f}$, где $n_{k}$ - наибольший нормальный индекс, не превосходящий $n$.

2.2. Строчные последовательности. Наиболее полно изучен вопрос о сходимости строк таблицы Паде (см. прежде всего [27]). Для произвольного целого $m \geqslant 0$ обозначим через $D_{m}=D_{m}(f)$ максимальный круг с центром в точке $z=0$, в который $f$ продолжается как мероморфная функция с числом полюсов $\leqslant m$ (полюсы и нули функции считаются с учетом их кратностей; аналитическое продолжение ряда $f$ также обозначаем через $f), R_{m}=R_{m}(f)$ радиус круга $D_{m}(f)$. Для произвольной точки $a \in \mathbb{C}^{*}:=\mathbb{C} \backslash\{0\}$ через $\mu(a)$ обозначим число полюсов функции $[n / m]_{f}$, стремящихся к $a$ со скоростью геометрической прогрессии. Положим $\Delta(a)=\varlimsup_{n \rightarrow \infty}\left|Q_{n, m}^{*}(a)\right|^{1 / n}$, где $Q_{n, m}^{*}-$ знаменатель аппроксимации Паде в сферической нормировке

$$
Q_{n, m}^{*}(\zeta)=\prod_{\left|\zeta_{n, j}\right| \leqslant 1}\left(\zeta-\zeta_{n, j}\right) \prod_{\left|\zeta_{n, j}\right|>1}\left(1-\frac{\zeta}{\zeta_{n, j}}\right)
$$

Пусть $\mathscr{P}_{m}:=\left\{a \in \mathbb{C}^{*}: \mu(a) \geqslant 1\right\}=\left\{a \in \mathbb{C}^{*}: \Delta(a)<1\right\}$. Тогда имеет место следующая теорема.

ТеОрема Гончара (см. [22], [27]). Последователъность $[n / m]_{f}$ сходится $\kappa f$ по емкости внутри (т. е. на компактных подмножествах) круга $D_{m}(f)$; радиус $R_{m}$ и дивизор $d_{m}$ полюсов функции $f$ в круге $D_{m}$ вичисляются по следующим формулам:

$$
R_{m}=\frac{|a|}{\Delta(a)} \quad \forall a \in \mathscr{P}_{m}, \quad d_{m}=\left\{(a, \mu(a)): a \in \mathscr{P}_{m}\right\} .
$$

При этом в $D_{m}$ имеет место представление:

$$
f(z)=\left[n_{0} / m\right]_{f}(z)+\sum_{k=n_{0}}^{\infty} \frac{A_{k, m}^{*} z^{k+m+1-\lambda_{k, m}}}{\left(Q_{k, m}^{*} Q_{k+1, m}^{*}\right)(z)},
$$

где равенство понимается в смысле сходимости по емкости, $\varlimsup_{k \rightarrow \infty}\left|A_{k, m}^{*}\right|^{1 / k}=$ $1 / R_{m}$; ряд в правой части (15) расходится вне $D_{m}$. Отсюда вытекает следующая теорема.

\footnotetext{
${ }^{7}$ Иногда нормальными называют именно те индексы $(n, m)$, для которых выполняется соотношение (11). Такое определение неудачно: оно неправильно отражает блочную структуру таблицы Паде степенного ряда и, кроме того, не может быть выражено в терминах определителей Адамара так же явно, как и общепринятое определение.
} 
Теорема Монтессу (см. [18], [27]). Если число полюсов $f$ в $D_{m}$ равно $m$, mо $[n / m]_{f} \rightarrow f$ в сферической метрике равномерно внутри $D_{m}$.

При этом неравенство

$$
\varlimsup_{n \rightarrow \infty}\left|\left(f-[n / m]_{f}\right)(z)\right|^{1 / n} \leqslant \frac{|z|}{R_{m}}<1, \quad z \in D_{m} \backslash\{\text { полюсы } f\},
$$

характеризует скорость этой сходимости.

Оригинальное доказательство (см. [18]) теоремы Монтессу было основано на теореме Адамара (см. [17]).

Теорема АДАмарА. Для произвольного $m \in \mathbb{N}$ справедлива следующая формула:

$$
R_{m}=\frac{\ell_{m}}{\ell_{m+1}}, \quad \text { əде } \quad \ell_{0}=1, \quad \ell_{j}=\varlimsup_{n \rightarrow \infty}\left|H_{n, j}\right|^{1 / n}, \quad j=1,2, \ldots
$$

$\left(\right.$ если $\ell_{1}, \ldots, \ell_{m} \neq 0, \ell_{m+1}=0$, mо $\left.R_{m}=\infty\right)$; здесъ $H_{n, m}$ - определитель Адамара (10).

Представление (15) означает, что использование аппроксимаций Паде приводит к нелинейному методу суммирования степенного ряда за пределами его круга сходимости (см. также (23)).

Отсутствие в общем случае равномерной сходимости $m$-й строки внутри круга $D_{m}$ даже по подпоследовательности вытекает из следующего примера. Для функции $f(z)=(1+\sqrt[3]{2} z) /\left(1-z^{3}\right)$ имеем: $D_{0}=D_{2}=\{z:|z|<1\}$, в то время как аппроксимация Паде $[n / 2]_{f}$ при каждом $n=0,1, \ldots$ имеет по меньшей мере один полюс в круге $|z| \leqslant 1 / \sqrt[3]{2}<1$. Аналогичные примеры известны и для любого $m \geqslant 3$ (см. [88]-[91]).

При условии, что $c_{n+1} \neq 0$, полюс $\zeta_{n}$ первой строки $[n / 1]_{f}$ таблицы Паде вычисляется по формуле $\zeta_{n}=c_{n} / c_{n+1}$. Хорошо известна следующая теорема.

ТЕОРЕма ФАБРИ “оБ отношЕНИи” (см. [92], [93]). Если для коэбфициентов степенного ряда $f=\sum_{k=0}^{\infty} c_{k} z^{k}$ выполняется соотношение

$$
\lim _{k \rightarrow \infty} \frac{c_{k}}{c_{k+1}}=a, \quad a \in \mathbb{C}^{*}
$$

mо $R_{0}=|a|$ и а-особая точка суммы степенного ряда $f(z)$.

Таким образом, если полюс первой строки таблицы Паде имеет предел $a \in$ $\mathbb{C}^{*}$, то по теореме Фабри об отношении имеем: $a$ - особая точка суммы степенного ряда $f(z),|z|<|a|$.

А. А. Гончар [27] высказал гипотезу, что аналогичное утверждение имеет место и для $m$-й строки таблицы Паде. Эта гипотеза была доказана С. П. Суетиным [94], [95].

Теорема Суетина. Если $Q_{n, m}=\prod_{j=1}^{m}\left(z-\zeta_{n, j}\right)$ для достаточно больших $n$, причем все $\zeta_{n, j}$ стремлтся $\kappa a_{j} \in \mathbb{C}^{*}$, где $0<\left|a_{1}\right| \leqslant \cdots \leqslant\left|a_{\mu-1}\right|<\left|a_{\mu}\right|=\cdots=$ $\left|a_{m}\right|=R$, mo: 
1) $R_{\mu-1}(f)=\cdots=R_{m-1}(f)=R$ и все точки $a_{1}, \ldots, a_{\mu-1}$ и только они полюсы функиии $f$ в круге $|z|<R$;

2) все точки $a_{\mu}, \ldots, a_{m}$ - особые точки функции $f$ на границе круга $|z|<R$.

2.3. Диагональные последовательности. При изучении диагональных аппроксимаций Паде обычно делают замену переменной $z \mapsto 1 / z$ и рассматривают ряд

$$
f=\sum_{k=0}^{\infty} \frac{c_{k}}{z^{k+1}} .
$$

Тогда $[n / n]_{f}=P_{n} / Q_{n}$, где полиномы $P_{n}, \operatorname{deg} P_{n} \leqslant n-1$, и $Q_{n}, \operatorname{deg} Q_{n} \leqslant n$, $Q_{n} \not \equiv 0$, определяются из соотношения

$$
\left(Q_{n} f-P_{n}\right)(z)=O\left(\frac{1}{z^{n+1}}\right), \quad z \rightarrow \infty
$$

(справа в (17) стоит ряд по убывающим степеням $z$ ).

Если $f=\widehat{\sigma}-$ марковская функция,

$$
\widehat{\sigma}(z):=\int \frac{d \sigma(x)}{z-x},
$$

где $\sigma$ - положительная борелевская мера с бесконечным компактным носителем $S_{\sigma}$ на $\mathbb{R}$, то: $c_{k}=\int x^{k} d \sigma(x)$ - моменты меры $\sigma$, все индексы $n$ нормальны, полиномы $Q_{n}$ ортогональны по мере $\sigma$ и условием $Q_{n}(z)=z^{n}+\cdots$ определены однозначно, аппроксимация Паде $[n / n]_{\widehat{\sigma}}$ совпадает с $n$-й подходящей дробью к $J-\partial р о б u^{8}$

$$
\widehat{\sigma}(z)=\frac{a_{0}^{2}}{z-b_{1}-\frac{a_{1}^{2}}{z-b_{2}-\frac{a_{2}^{2}}{z-b_{3}-\cdot}}}
$$

$\left(a_{0}^{2}=c_{0}=|\sigma|, a_{k}, b_{k} \in \mathbb{R}, k=1,2, \ldots\right)$, которая строится по ряду (16) с помощью алгоритма Евклида. Для функции $\widehat{\sigma}$ все полюсы аппроксимации Паде $[n / n]_{\widehat{\sigma}}$ лежат на отрезке $[A, B],[A, B]=\widehat{S}_{\sigma}-$ выпуклая оболочка $S_{\sigma}$.

Теорема Маркова [4] (см. также [27], [11] и раздел 1 Введения) утверждает, что непрерывная дробъ (19) сходится $\kappa \widehat{\sigma}$ в $\overline{\mathbb{C}} \backslash[A, B]$, при этом скорость сходимости характеризуется неравенством:

$$
\varlimsup_{n \rightarrow \infty}\left|\left(\widehat{\sigma}-[n / n]_{\widehat{\sigma}}\right)(z)\right|^{1 / n} \leqslant \frac{1}{|\Phi(z)|^{2}}, \quad z \notin[A, B],
$$

где функция $\Phi$ отображает конформно и однолистно внешность $[A, B]$ на внешность единичного круга, $\Phi(\infty)=\infty$.

Если $S_{\sigma}=[-1,1]-$ отрезок, $\sigma^{\prime}=d \sigma / d x>0$ почти всюду на $[-1,1]$, то в $(20)$ имеет место точное равенство, а верхний предел превращается в обычный. При этом условии на $\sigma$ аналог теоремы Маркова имеет место для функций вида

\footnotetext{
${ }^{8}$ Дробь (19) иногда называют чебышёвской непрерывной дробъю; см. [3].
} 
$f=\widehat{\sigma}+r$, где $r$ - рациональная функция с комплексными коэффициентами, не имеющая полюсов на $[-1,1], r(\infty)=0(r$ - "рациональное возмущение" марковской функции $\widehat{\sigma}$; см. [27], а также раздел 1 главы 1).

Теорема Гончара (см. [27]). Если $f=\widehat{\sigma}+r$, mo $[n / n]_{f} \rightarrow f$ в сферической метрике внутри (на компактных подмножествах) области $D=\overline{\mathbb{C}} \backslash[-1,1]$.

Тем самым, каждый полюс $r$ притягивает ровно столько полюсов $[n / n]_{f}$, какова его кратность, остальные полюсы $[n / n]_{f}$ притягиваются к отрезку $[-1,1]$. В предположении, что $d \sigma(x)=\left(1-x^{2}\right)^{-1 / 2} \rho(x) d x$, где $\rho-$ комплексная голоморфная функция на $[-1,1], \rho(x) \neq 0$, поведение $m$ полюсов $[n / n]_{f}$ в малой окрестности $m$-кратного, $m \geqslant 3$, полюса $r$ уточняется следующим образом: асимптотически они располагаются в вершинах правильного т-угольника (см. [96], а также [97]). Приведенные результаты справедливы для простейших алгебраических функций вида $f(z)=r_{1}(z) / \sqrt{z^{2}-1}+r_{2}(z), z \in D$, при надлежащих ограничениях на рациональные функции $r_{1}, r_{2}$.

Если $S_{\sigma}$ состоит из нескольких непересекающихся отрезков, то в лакунах между ними располагаются “ложсные" полюсы аппроксимаций Паде $[n / n]_{\widehat{\sigma}}$, которые в "типичном случае" (и при надлежащих ограничениях на меру $\sigma$ ) всюду плотны в этих лакунах (см. [98]-[100]). Аналогичное утверждение справедливо и для так называемых "дополнительных" точек интерполяции (см. [99]). Динамика таких полюсов аппроксимаций Паде и точек интерполяции подчиняется следующей нелинейной системе дифференциальных уравнений Дубровина относительно набора точек $\mathbf{z}_{1}(t), \ldots, \mathbf{z}_{g}(t), t \in \mathbb{R}_{+}$, расположенных на соответствующей гиперэллиптической римановой поверхности $\mathfrak{R}$ рода $g$ :

$$
\dot{z}_{k}=-\frac{2 w\left(\mathbf{z}_{k}\right)}{\prod_{j \neq k}\left(z_{k}-z_{j}\right)} \int_{e_{2 g+2}}^{\infty} \frac{\prod_{j \neq k}\left(x-z_{j}\right)}{w(x)} d x, \quad k=1, \ldots, g,
$$

где $\dot{z}_{k}=d z_{k} / d t, e_{2 g+2}$ - крайняя правая точка носителя меры $\sigma$ (предполагается, что носитель меры $\sigma$ имеет $g \geqslant 1$ лакун, краевые точки отрезков носителя занумерованы в порядке возрастания их величин, а путь интегрирования в $(21)$ - часть вещественной оси $\left[e_{2 g+2},+\infty\right)$; подробнее по поводу обозначений см. [99]). Если $r$ - вещественная рациональная функция, все полюсы которой лежат вне $\widehat{S}_{\sigma}$, то для $f=\widehat{\sigma}+r$ имеем: $[n / n]_{f} \rightarrow f$ в сферической метрике локалъно равномерно в $\overline{\mathbb{C}} \backslash \widehat{S}_{\sigma}$; тем самым, каждый полюс $r$ притягивает ровно столько полюсов $[n / n]_{f}$, какова его кратность (см. [43]). В этом случае динамика полюсов и точек интерполяции аппроксимаций Паде $[n / n]_{f}$, лежащих в лакунах между отрезками, также подчиняется системе (21). Если $r-$ комплексная рациональная функция, то полюсы $[n / n]_{f}$ могут иметь предельные точки вне $\widehat{S}_{\sigma}$, отличные от полюсов $r$; в этом случае $[n / n]_{f} \rightarrow f$ внутри $\overline{\mathbb{C}} \backslash S_{\sigma}$ по емкости; тем самым, каждый полюс $r$ притягивает по меньшей мере столько полюсов $[n / n]_{f}$, какова его кратность (см. [43]).

В [101] (см. также [102]) исследован вопрос об асимптотическом поведении аппроксимаций Паде для эллиптической ${ }^{9}$ функции вида

$$
f(z)=\sqrt{\left(z-e_{1}\right) \cdots\left(z-e_{4}\right)}-z^{2}+\frac{e_{1}+\cdots+e_{4}}{2} z,
$$

\footnotetext{
${ }^{9}$ Под эллиптическими функциями здесь понимаются функции, принадлежащие кольцу алгебраических функций на эллиптической римановой поверхности; см., например, [103].
} 
где точки $e_{1}, \ldots, e_{4} \in \mathbb{C}$ попарно различны и выбрана та ветвь корня, старший член которой в окрестности бесконечно удаленной точки равен $z^{2}$. Оказалось, что асимптотическое поведение диагональных аппроксимаций Паде $[n / n]_{f}$ зависит от расположения точек $e_{1}, \ldots, e_{4}$; если эти точки находятся в "общем положении", то множество полюсов функиий $[n / n]_{f}$ всюду плотно в $\overline{\mathbb{C}}$, т. е. последовательность $[n / n]_{f}$ не может равномерно сходиться ни в какой подобласти $\mathbb{C}$.

Исследование сходимости аппроксимаций Паде для общих гиперэллиптических функций вида $f=r_{1}(z) / \sqrt{h(z)}+r_{2}(z)$, где $h-$ полином четной степени $2 p$ с простыми нулями $e_{1}, \ldots, e_{2 p} \in \mathbb{C}$, приводит (см. [35], [36], [40]) к понятию компакта минимальной емкости $S=S(h)$ для многозначной функции $\sqrt{h(z)}$ : $\sqrt{h(z)}$ продолжается из окрестности точки $z=\infty$ в дополнение к $S$ как однозначная аналитическая функция, а из всех компактов, обладающих таким свойством, $S$ имеет наименьшую емкость. Если все $e_{1}, \ldots, e_{2 p} \in \mathbb{R}$, то $S$ состоит из $p$ отрезков, расположенных на $\mathbb{R}$. Последовательность $[n / n]_{f}$ сходится $\kappa f$ по емкости внутри $\overline{\mathbb{C}} \backslash S$; тем самым, каждый полюс $f$ притягивает по меньшей мере столько полюсов $[n / n]_{f}$, какова его кратность. Этот результат остается справедливым и в классе функций $f=\widehat{\sigma}$, где

$$
\widehat{\sigma}(z):=\int \frac{d \sigma(t)}{z-t}, \quad z \notin S_{\sigma},
$$

$S_{\sigma}=S(h)$, а комплексная мера $\sigma$ имеет вид $d \sigma(z)=\rho(z) / \sqrt{h(z)}_{+}, z \in S$, где функция $\rho(z)$ не обращается в нуль и удовлетворяет определенному условию регулярности (см. [36], [40], а также раздел 2 главы 1).

Наиболее общий результат прямого характера о сходимости диагональных аппроксимаций Паде - теорема Шталя [63]-[67] (см. также [69], [104] и раздел 3 главы 1), из которой, в частности, следует, что если ряд $f$ соответствует алгебраической функции, то $[n / n]_{f} \rightarrow f$ по емкости внутри области $\overline{\mathbb{C}} \backslash F$, где $F=F(f)$ - компакт минимальной емкости, соответствующий выбранной рядом $f$ ветви алгебраической функции: $f$ продолжается из окрестности точки $z=\infty$ в дополнение к $F$ как однозначная аналитическая функция, а из всех компактов, обладающих таким свойством, $F$ имеет наименьшую емкость. Компакт $F$ не разбивает плоскость, состоит из конечного числа кусочно аналитических дуг и вполне характеризуется определенным свойством "симметрии" (или $S$-свойством). Область Шталя $D:=\overline{\mathbb{C}} \backslash F, D=D(f)$, является максимальной (по включению) областъю сходимости диагональных аппроксимаций Паде $[n / n]_{f}$ алгебраической функции $f$, при этом в области Шталя $D$ справедливо представление

$$
f(z)=\left[n_{0} / n_{0}\right]_{f}(z)+\sum_{k=n_{0}}^{\infty} \frac{A_{k}}{\left(Q_{k} Q_{k+1}\right)(z)},
$$

где равенство понимается в смысле сходимости по емкости. Сходимость аппроксимаций Паде и представление (23) имеют место и для функций вида $f=\widehat{\sigma}+r$, где $S_{\sigma}=F$ - симметричный компакт, а мера удовлетворяет условию $d \sigma / d s \neq 0$ почти всюду на $F(d s-$ элемент длины дуги $F)$. При этом каждый полюс $r$ в $D$ притягивает по меньшей мере столько полюсов $[n / n]_{f}$, какова 
его кратность, и если $\mu_{n}$ - нормированная считающая мера нулей знаменателя $[n / n]_{f}$, то $\mu_{n} \rightarrow \lambda_{F}$, где $\lambda_{F}-$ равновесная мера компакта $F$.

Отсутствие равномерной сходимости диагональных аппроксимаций Паде внутри области Шталя $D=\overline{\mathbb{C}} \backslash F$ даже по подпоследовательности вытекает из следующего примера В.И. Буслаева (см. [105], [106], а также п. 6.2 главы 1). Пусть $\beta(\zeta)=\left(p_{3}(\zeta)+\sqrt{h_{6}(\zeta)}\right) / q_{3}(\zeta)$, где $p_{3}(\zeta)=-27+6 \zeta^{2}+3(9+\varepsilon) \zeta^{3}$, $q_{3}(\zeta)=2 \zeta\left(9+9 \zeta+(9+\varepsilon) \zeta^{2}\right), h_{6}(\zeta)=81\left(3-(3+\varepsilon) \zeta^{3}\right)^{2}+4 \zeta^{6}, \varepsilon=\exp (2 \pi i / 3)$, ветвь корня выбрана так, что функция $\beta$ голоморфна в нуле, при этом $\beta(0)=0$. Тогда для гиперэллиптической (рода 2) функции $\mathfrak{B}(z)=\beta(1 / z)$ симметричный компакт $F=F(\mathfrak{B})$ является собственным подмножеством $U_{1}=\{z:|z|<1\}$, функция $\mathfrak{B}$ мероморфна в области $D=\overline{\mathbb{C}} \backslash F$ и имеет единственный простой полюс в точке $z=a \in U_{1}$; тем самым, $\mathfrak{B}$ голоморфна во внешности единичного круга. В то же время диагональная аппроксимация Паде $[n / n]_{\mathfrak{B}}$ при каждом $n=1,2, \ldots$ имеет полюс, лежашиц во внешности круга $U_{1}$. Этот пример опровергает так называемую "Паде-гипотезу" (см. [8; ч. 2, гл. 6, $\S 6.7])$; первый контрпример к этой гипотезе, основанный на непрерывной дроби Роджерса-Рамануджана, был получен Д. Любински в 2001 г. (см. [107]).

$\mathrm{C}$ диагональными аппроксимациями Паде функции $\mathfrak{B}$ связан следующий эффект: при каждом $n$ функция $[n / n]_{\mathfrak{B}}$ в некоторой точке $z_{n} \in \mathbb{C} \backslash \bar{U}_{1}$ интерполирует другую ветвъ $\mathfrak{B}^{*}(z)=\left(p_{3}(\zeta)-\sqrt{h_{6}(\zeta)}\right) / q_{3}(\zeta)$ функиии $\mathfrak{B}$. Это явление "ложной интерполяции" носит достаточно общий и вполне естественный характер и имеет место, например, для эллиптических функций [108]. Возникновение у аппроксимаций Паде ложных полюсов связано именно с ложной интерполяцией. Для эффективного использования аппроксимаций Паде при вычислениях разработаны “сглаживающие" процедуры, учитывающие возможное возникновение ложных полюсов (см., например, [109], [110]).

Принципиальный результат обратного характера получен А. А. Гончаром (см. [28], [29], а также раздел 5 главы 1). Пусть область $G \ni \infty$ - дополнение к выпуклому компакту, $e \not \ngtr \infty$ - относительно замкнутое в $G$ множество нулевой емкости, $D=G \backslash e$. Если множество полюсов диагональных аппроксимаций ряда $f$ не имеет предельных точек в области $D$, то $[n / n]_{f} \rightarrow f$ локально равномерно в $D$; тем самым, ряд $f$ продолжается из окрестности точки $z=\infty$ в область $D$ как однозначная аналитическая функция и имеет место представление (23). Таким образом, голоморфность диагональных аппроксимаций Паде в областях указанного вида является критерием их равномерной сходимости. Теорема Маркова является непосредственным следствием этого критерия. Аналогичный критерий имеет место и для равномерной сходимости в сферической метрике. Отметим, что из классических результатов о нормальных семействах мероморфных функций (теорема Монтеля) вытекает локально равномерная сходимость последовательности $\left\{\Phi_{n}\right\}$ в заданной области $D$ в том случае, когда функции $\Phi_{n}$ этой последовательности в области $D$ выпускают mpu значения $a, b, c=\infty$. Результат А. А. Гончара показывает, что последовательности диагональных аппроксимаций Паде имеют замечательную специфику - их равномерная сходимость (в областях указанного выше вида) вытекает уже из того факта, что функции $[n / n]_{f}$ выпускают в области $D$ только одно значение $c=\infty$ (не имеют полюсов). 
2.4. Некоторые замечания. К настоящему времени аппроксимации Паде нашли многочисленные применения в различных задачах теоретической и прикладной математики, теоретической и математической физики, механики (см. прежде всего [8], [9], а также [111]-[119]). В свою очередь, многие замечательные свойства аппроксимаций Паде были открыты сначала эмпирически в результате численных расчетов и лишь затем строго обоснованы. Изучение возникающих при этом математических проблем привело к новым задачам и результатам в комплексном анализе, теории потенциала, теории ортогональных многочленов (см. [11], [8], [12]). В последнее время методы, развитые в теории аппроксимаций Паде, получили широкое применение при исследовании спектральных свойств оператора Якоби и различных ансамблей случайных матриц (см. прежде всего [58], а также [54], [60]-[62], [120]-[131]).

Для практического вычисления аппроксимаций Паде обычно пользуются рекуррентными соотношениями, существующими в таблице Паде. На основе этих соотношений разработано много алгоритмов для численного нахождения аппроксимаций Паде (см. [8], а также [118], [119]).

Рассматривают различные обобщения классических аппроксимаций Паде: многоточечные аппроксимации Паде, аппроксимации Паде ортогональных разложений, рядов Фабера, тригонометрических рядов, аппроксимации ЭрмитаПаде и др. (см. [8], [11], [12], [26], [30], [31], [97], [123], [124], [132]-[139]). Во всех этих случаях речь идет о приближениях рациональными функциями со свободными полюсами, а сами рассматриваемые аппроксимации имеют нелинейныи характер. "Оптимальный" выбор коэффициентов как числителей, так и знаменателей этих аппроксимаций позволяет с их помощью успешно решать задачу “эффективного аналитического продолжения" 10 локально заданной функции (см. (15), (23)). В этом состоит принципиальное отличие таких рациональных аппроксимаций от полиномиальных приближений и от рациональных аппроксимаций с заранее фиксированными полюсами.

\section{Глава 1}

\section{Асимптотические свойства ортогональных многочленов и сходимость аппроксимаций Паде}

\section{1. Сходимость аппроксимаций Паде для функций марковского типа}

1.1. Введение. Пусть

$$
f=\sum_{k=0}^{\infty} \frac{c_{k}}{z^{k+1}}
$$

- степенной ряд с центром в бесконечно удаленной точке $z=\infty, f_{n}=[n / n]_{f}$, $n=1,2, \ldots,-$ последовательность его диагональных ${ }^{11}$ аппроксимаций Паде.

\footnotetext{
${ }^{10}$ По поводу этого понятия см. [140].

${ }^{11}$ Термин "диагональная последовательность" связан с тем, что такие аппроксимации составляют главную диагональ в общей таблище Паде ряда $f$ (см. [8], а также раздел 2 главы 1).
} 
В случае, когда все ганкелевы определители

$$
H_{n}=\left|\begin{array}{cccc}
c_{0} & c_{1} & \ldots & c_{n} \\
c_{1} & c_{2} & \ldots & c_{n+1} \\
\ldots \ldots & \ldots \ldots \ldots & \ldots \\
c_{n} & c_{n+1} & \ldots & c_{2 n}
\end{array}\right|, \quad n=0,1, \ldots
$$

отличны от нуля, ряду (24) с помощью классического алгоритма Евклида сопоставляется $J$-дробь (или, иначе говоря, чебышёвская непрерывная дробь; cм. [3], [8]):

$$
f \sim \frac{a_{0}^{2}}{z-b_{1}-\frac{a_{1}^{2}}{z-b_{2}-\cdot}},
$$

$n$-я подходящая дробь которой совпадает с $f_{n}$ и обладает следующим характеристическим свойством:

$$
\left(f-f_{n}\right)(z)=\frac{A_{n}}{z^{2 n+1}}+\cdots
$$

где $A_{n} \neq 0$. Тем самым, дробь (25) есть просто другая (эквивалентная) форма записи для последовательности $\left\{f_{n}\right\}_{n \in \mathbb{N}}$.

В общем случае удобно основываться на следующем определении $n$-й диагональной аппроксимации Паде ряда $f$ (определение Фробениуса): $f_{n}=P_{n} / Q_{n}$, где $P_{n}, Q_{n}$ - полиномы от $z, \operatorname{deg} P_{n}<\operatorname{deg} Q_{n} \leqslant n\left(Q_{n} \not \equiv 0\right)$ и выполняется соотношение

$$
R_{n}(z):=\left(Q_{n} f-P_{n}\right)(z)=O\left(\frac{1}{z^{n+1}}\right), \quad z \rightarrow \infty .
$$

Этими условиями полиномы $P_{n}$ и $Q_{n}$ определяются не однозначно, но рациональная дробь $f_{n}=P_{n} / Q_{n}$ единственна (с точностью до стандартного отождествления). Функция $R_{n}$ называется функцией остатка. Для нормальных индексов $n$ порядок рациональной функции $f_{n}$ равен $n$. Тем самым, для таких $n$ полиномы $P_{n}$ и $Q_{n}$, удовлетворяющие условию $(27)$, не имеют общих нулей, $\operatorname{deg} Q_{n}=n$ и выполняется соотношение (26) (где, возможно, $A_{n}=0$ ). Каково бы ни было $n$, справедливо равенство $f_{n}=f_{n_{k}}$, где $n_{k}$ - наибольший нормальный индекс, не превосходящий $n$. Тем самым, последовательность нормальных индексов $\left\{n_{k}\right\}=\Lambda=\Lambda(f)$ для ряда $f$ разбивает натуральный ряд чисел на множества $\Lambda_{k}=\left\{n: n_{k} \leqslant n<n_{k+1}\right\}$ такие, что $f_{n}=f_{n_{k}}$ для $n \in \Lambda_{k}$. Такая структура последовательности $\left\{f_{n}\right\}_{n \in \mathbb{N}}$ позволяет в вопросах сходимости диагональных аппроксимаций Паде ограничиться рассмотрением последовательности $\left\{f_{n}\right\}_{n \in \Lambda}$.

Для марковской функции

$$
\widehat{\sigma}(z):=\int \frac{d \sigma(x)}{z-x}, \quad z \in \overline{\mathbb{C}} \backslash S,
$$

где $\sigma$ - положительная борелевская мера с носителем $S=\operatorname{supp} \sigma \Subset \mathbb{R}$, коэффициенты соответствующего ей ряда (24) совпадают с моментами меры $\sigma$ :

$$
c_{n}=\int x^{n} d \sigma(x), \quad n=0,1,2, \ldots .
$$


При этом все определители $H_{n}$ положительны, полиномы $Q_{n}(z)=Q_{n}(z ; \widehat{\sigma})$ имеют степень $n$ и при нормировке $Q_{n}(z ; \widehat{\sigma})=z^{n}+\cdots$ определяются единственным образом:

$$
Q_{n}(z ; \widehat{\sigma})=\frac{1}{H_{n-1}}\left|\begin{array}{cccc}
c_{0} & c_{1} & \ldots & c_{n} \\
c_{1} & c_{2} & \ldots & c_{n+1} \\
\ldots \ldots & \ldots \ldots \ldots & \ldots \ldots \\
c_{n-1} & c_{n} & \ldots & c_{2 n-1} \\
1 & z & \ldots & z^{n}
\end{array}\right|=z^{n}+\cdots, \quad n=1, \ldots
$$

Полиномы $Q_{n}$ ортогональны по мере $\sigma$, при этом для функции остатка $(27)$ справедливо представление

$$
R_{n}(z)=\int \frac{Q_{n}(x) d \sigma(x)}{z-x}, \quad z \in \widehat{\mathbb{C}} \backslash S,
$$

а для полиномов $P_{n}-$ представление

$$
P_{n}(z)=\int \frac{Q_{n}(z)-Q_{n}(x)}{z-x} d \sigma(x)
$$

в теории ортогональных многочленов (29) принято называть функцией второго рода, а (30) - многочленами второго рода. Отметим, что все три функции $Q_{n}, R_{n}$ и $P_{n}$ удовлетворяют одному и тому же рекуррентному соотношению

$$
X_{n}(z)=\left(z-b_{n}\right) X_{n-1}(z)-a_{n-1}^{2} X_{n-2}(z), \quad n=2,3, \ldots,
$$

но с разными начальными условиями $X_{0}, X_{1}$.

Классическая теорема Маркова [4] (см. также [141]-[145]) утверждает, что для марковской функции $\widehat{\sigma}$ чебышёвская непрерывная дробъ (25) равномерно сходится внутри (на компактных подмножествах) области $D=\widehat{\mathbb{C}} \backslash[\alpha, \beta]$, где $[\alpha, \beta]$ - выпуклая оболочка $S_{\sigma}$.

1.2. В 1975 г. А. А. Гончар получил обобщение теоремы Маркова на класс мероморфных функций вида

$$
f=\widehat{\sigma}+r
$$

где $r$ - рациональная функция с комплексными коэффициентами $(r$ - "рациональное возмущение" марковской функции $\widehat{\sigma})$.

Теорема 1 (Гончар [21]). Пусть $\sigma$ - положительная борелевская мера с носителем $\operatorname{supp} \sigma=[\alpha, \beta], \sigma^{\prime}>0$ почти всюду на $[\alpha, \beta]^{12} u f=\widehat{\sigma}+r$, где $r$ произвольная комплексная рациональная функция, все полюсы которой принадлежат области $\mathbb{C} \backslash[\alpha, \beta], u r(\infty)=0$. Тогда последовательность диагональных аппроксимаций Паде $\left\{f_{n}\right\}_{n \in \mathbb{N}}$ сходится $\kappa f$ равномерно в сферической метрике ${ }^{13}$ внутри области $D=\widehat{\mathbb{C}} \backslash[\alpha, \beta]$.

${ }^{12} \mathrm{~B}[21]$ мера $\sigma$ предполагалась такой, что имеет место асимптотика отношения многочленов, ортогональных на $[\alpha, \beta]$ относительно $\sigma$. Позднее Е. А. Рахмановым [146], [147] было показано, что при условии: $\sigma^{\prime}>0$ почти всюду на $[\alpha, \beta]$, эта асимптотика отношения имеет место.

${ }^{13}$ Имеется в виду, что за расстояние между двумя точками в комплексной плоскости принято расстояние между соответствующими точками на сфере Римана. 
Из теоремы 1 вытекает, что к каждому полюсу функции $f$ в $D$ стремится (со скоростью геометрической прогрессии; см. ниже формулу (34)) ровно столько полюсов $f_{n}$, какова его кратность, а все предельные точки остальных полюсов лежат на отрезке $[\alpha, \beta]$. Обратим внимание, что по отмеченной в конце введения общей теоремы Гончара из указанного асимптотического поведения полюсов диагональных аппроксимаций Паде для формального ряда $f$ уже вытекает равномерная сходимость последовательности $f_{n}$ внутри области $\widehat{\mathbb{C}} \backslash[\alpha, \beta]$ (в сферической метрике); тем самым, этот ряд определяет аналитическую функцию $f$, допускающую мероморфное продолжение во внешность отрезка $[\alpha, \beta]$.

Таким образом, диагональные аппроксимации Паде в классе функций вида (32), заданных своими разложениями в степенной ряд (24), полностью решают задачу эфбективного аналитического продолжения степенного ряда в область $D=D_{f}$ : полюсы этих аппроксимаций локализуют как отрезок $[\alpha, \beta]=$ $\operatorname{supp} \sigma$, так и полюсы $f$ в $D$ с учетом их кратностей, а сами аппроксимации $f_{n}$ восстанавливают $f$ внутри $D$.

В [21] указана также скорость сходимости последовательности $f_{n}$ к функции $f$ в "проколотой" области $D^{\prime}=D \backslash\{$ полюсы $f\}$ :

$$
\varlimsup_{n \rightarrow \infty}\left|\left(f-f_{n}\right)(z)\right|^{1 / n} \leqslant \frac{1}{|\Phi(z)|^{2}},
$$

где $\Phi$ - функция, отображающая внешность отрезка $[\alpha, \beta]$ на внешность единичного круга с условием $\Phi(\infty)=\infty$.

Отсюда уже вытекает $\left[22 ; \S 2\right.$, п. 10], что полюсы $f_{n}$ стремятся к полюсам $f$ в $D$ со скоростъю геометрической прогрессии; при этом, если $a$ - полюс $f$ кратности $\mu \geqslant 1$, то

$$
\varlimsup_{n \rightarrow \infty}\left|a-\zeta_{j, n}(a)\right|^{1 / n} \leqslant \frac{1}{|\Phi(a)|^{2 / \mu}}, \quad j=1, \ldots, \mu, \quad|\Phi(a)|>1 ;
$$

здесь $\zeta_{j, n}(a), j=1, \ldots, \mu,-$ полюсы ${ }^{14} f_{n}$, перенумерованные в порядке неубывания их расстояния до точки $a$.

В работе [21; $\$ 2$, п. 11] было отмечено, что как теорема 1 , так и ее следствия остаются в силе для любых мероморфных в области $D$ функций $f$ (с конечным числом полюсов в $D$ ), голоморфные составляющие $f^{*}$ которых могут быть представлены в виде

$$
f^{*}(z)=\int_{\alpha}^{\beta} \frac{s(x) d \sigma(x)}{z-x}, \quad z \in D
$$

где $s$ - произвольная комплексная рациональная функция, все нули и полюсы которой принадлежат области $D$. В частности, это относится и к простейшим алгебраическим функциям вида

$$
f(z)=\frac{r_{1}(z)}{w}+r_{2}(z)
$$

\footnotetext{
${ }^{14}$ Как обычно, полюсы и нули функций считаются с учетом их кратностей.
} 
где $w=\sqrt{(z-\alpha)(z-\beta)}$, при условии, что все полюсы рациональных функций $r_{1}, r_{2}$ лежат в $D, r_{1}$ не имеет нулей на отрезке $[\alpha, \beta]$ и в $D$ зафиксирована определенная ветвь квадратного корня.

В условиях теоремы 1 для функций вида (32) в (33) и (34) имеет место точное равенство; тем самым, в правой части (34) указан правильный показатель скорости сходимости полюсов $f_{n}$ к полюсам $f$ в области $D$. В то же время соотношения такого типа дают информацию о поведении каждого из $\mu$ полюсов аппроксимаций Паде по отдельности и не позволяют судить о том, какова "в целом" картина поведения $\mu$ полюсов $f_{n}$ в окрестности $\mu$-кратного полюса $f$. В работе [96] в предположении, что $d \sigma(x)=\left(1-x^{2}\right)^{-1 / 2} \rho(x) d x$, где $\rho$ - комплексная голоморфная функция на $[-1,1], \rho(x) \neq 0$, поведение $\mu$ полюсов аппроксимаций Паде в малой окрестности $\mu$-кратного, $\mu \geqslant 3$, полюса функции $f$ уточняется следующим образом: асимптотически все они простые и располагаются в вершинах правильного н-угольника. Приведенные результаты справедливы для простейших алгебраических функций вида (36) при надлежащих ограничениях на рациональные функции $r_{1}, r_{2}$.

Доказательство теоремы 1 основано на следующей полученной в [21] формуле сравнительной асимптотики ортогональных многочленов $Q_{n}(z ; \widehat{\sigma})$ и $Q_{n}(z ;$ $\widehat{\sigma}+r)$, которая выполняется равномерно внутри $D$ :

$$
\lim _{\substack{n \rightarrow \infty \\ n \in \Lambda}} \frac{Q_{n}(z ; \widehat{\sigma}+r)}{Q_{n}(z ; \widehat{\sigma})}=\prod_{j=1}^{\ell}\left(\frac{\Phi(z)-\Phi\left(a_{j}\right)}{1-\Phi(z) \Phi\left(a_{j}\right)}\right)^{m_{j}},
$$

где $\Lambda=\Lambda(f)$ - последовательность нормальных индексов для $f=\widehat{\sigma}+r$, $a_{1}, \ldots, a_{\ell}-$ полюсы $f$ в $D, m_{1}, \ldots, m_{\ell}-$ их кратности, $m=\sum_{j=1}^{\ell} m_{j}-$ полное
число полюсов $f$ в $D$.

1.3. Пусть $\mathfrak{R}$ - риманова поверхность, заданная уравнением $w^{2}=z^{2}-1$. Будем считать, что $\mathfrak{R}$ реализована как двулистное разветвленное в точках $z= \pm 1$ накрытие расширенной комплексной плоскости $\overline{\mathbb{C}}$. Два листа $\mathfrak{R}-$ это два экземпляра $\widehat{\mathbb{C}}$, разрезанных по отрезку $\Delta$ и склеенных затем накрест так, что верхнему берегу одного отрезка соответствует нижний берег другого. Тем самым, над каждой точкой $\widehat{\mathbb{C}}$ за исключением точек ветвления $z= \pm 1$ лежат ровно две точки римановой поверхности, а отрезку $\Delta$ соответствует на $\mathfrak{R}$ замкнутый цикл $\Gamma$. Будем считать, что в $D=\widehat{\mathbb{C}} \backslash \Delta$ выбрана ветвь корня $\sqrt{z^{2}-1}$, удовлетворяющая условию $\sqrt{z^{2}-1} / z \rightarrow 1$ при $z \rightarrow \infty$. Функция $w: w^{2}=z^{2}-1$ однозначна на $\mathfrak{R}$. Первым (открытым) листом $D^{(1)}$ поверхности $\mathfrak{R}$ будем считать тот, на котором $w=\sqrt{z^{2}-1}$. На втором листе $D^{(2)}$ имеем: $w=-\sqrt{z^{2}-1}$. Для точек римановой поверхности $\mathfrak{R}$ будем использовать обозначение $\mathbf{z}=(z, w)$, где $w= \pm \sqrt{z^{2}-1} ;$ при этом для точек первого листа $z^{(1)}=\left(z, \sqrt{z^{2}-1}\right)$, а для точек второго $z^{(2)}=\left(z,-\sqrt{z^{2}-1}\right)$. Область $D^{(1)}$ будем, как правило, отождествлять с "физической” областью $D$. Пусть $\Phi(z)=z+\sqrt{z^{2}-1}-$ функция, обратная к функции Жуковского. Тогда $\Phi$ однозначна в $D$ и продолжается на всю риманову поверхность $\mathfrak{R}$ как однозначная мероморфная функция, $\Phi(\mathbf{z})=z+w \in \mathbb{C}(z, w)$, с дивизором $(\Phi)=\infty^{(2)}-\infty^{(1)}$.

Зафиксируем произвольное $n \in \mathbb{N}$ и рассмотрим на двулистной римановой поверхности $\mathfrak{R}: w^{2}=z^{2}-1$ следующую краевую задачу Римана. 
ЗАДАчА 1. При биксированном $n \in \mathbb{N}$ найти функцию $\Psi=\Psi_{n}$ такую, что:

(i) $\Psi$ (кусочно) мероморфна на $\Re \backslash \Gamma=D^{(1)} \sqcup D^{(2)}$;

(ii) дивизор $(\Psi)=n \infty^{(2)}-n \infty^{(1)}$;

(iii) на $\mathscr{L}$ выполнено краевое условие: $\rho(x) \Psi^{(1)}(\mathbf{x})=\Psi^{(2)}(\mathbf{x})$.

Здесь под $\Psi^{(1)}(\mathbf{x})$ понимаются предельные значения функции $\Psi\left(\zeta^{(1)}\right)$ при $D^{(1)} \ni \zeta^{(1)} \rightarrow \mathbf{x} \in \mathscr{L}$. Аналогичный смысл придается и $\Psi^{(2)}(\mathbf{x})$.

Из свойств $\Psi$-функции вытекает, что $\Psi_{n}\left(z^{(1)}\right) \Psi_{n}\left(z^{(2)}\right) \equiv$ const $\neq 0$ при $z \in D$. Тем самым, это произведение продолжается как постоянная функция на всю комплексную плоскость $\overline{\mathbb{C}}$. В дальнейшем нам удобно ввести следующую нормировку ${ }^{15}: \Psi_{n}\left(z^{(1)}\right) \Psi_{n}\left(z^{(2)}\right) \equiv 1$ при $z \in D$.

Так как род $\mathfrak{R}$ равен нулю, то задача 1 распадается на две независимые задачи, соответствующие п. (ii) и п. (iii). Стандартными методами доказывается, что такая функция $\Psi$ всегда существует и определяется однозначно с точностью до мультипликативной постоянной. Нетрудно найти и явный вид этой функции:

$$
\Psi_{n}(z)=\Phi(z)^{n} V(z ; \rho)=e^{n G(z)} V(z ; \rho) \quad \text { при } \mathbf{z}=z^{(1)},
$$

где $\Psi_{n}(z):=\Psi_{n}\left(z^{(1)}\right)$,

$$
V(z ; \rho)=\{D(z ; \rho)\}^{-1}, \quad D(z: \rho)=\exp \left\{\frac{\sqrt{z^{2}-1}}{2 \pi} \int_{\Delta} \frac{\log \rho(x)}{z-x} \frac{d x}{\sqrt{1-x^{2}}}\right\},
$$

- функция Сегё:

$$
D^{+}(x ; \rho) D^{-}(x ; \rho) \equiv \rho(x), \quad x \in \Delta,
$$

для функции $V(z ; \rho)$ имеем

$$
\rho(x) V^{+}(x ; \rho) V^{-}(x ; \rho) \equiv 1, \quad x \in \Delta,
$$

тем самым,

$$
\rho(x) \Psi_{n}^{+}(x) \Psi_{n}^{-}(x) \equiv 1, \quad x \in \Delta .
$$

Соотношение $\Psi_{n}\left(z^{(1)}\right) \Psi_{n}\left(z^{(2)}\right) \equiv 1$ при $z \in D$ в совокупности с (38)-(39) приводит к явной формуле для $\Psi$-функции и при $\mathbf{z}=z^{(2)} \in D^{(2)}$; имеем

$$
\Phi\left(z^{(1)}\right) \Phi\left(z^{(2)}\right) \equiv 1, \quad D\left(z^{(2)} ; \rho\right) \equiv\left\{D\left(z^{(1)} ; \rho\right)\right\}^{-1} .
$$

При подходящей нормировке полиномов $Q_{n}(z ; \widehat{\rho})$ - знаменателей диагональных аппроксимаций Паде функции $f=\widehat{\rho}$ - их сильная асимптотика как вне отрезка $\Delta$, так и на этом отрезке описывается в терминах $\Psi$-функции следующим образом (см. [96]).

ТЕорема 2. Пусть комплексная функиия $\rho$ голоморфна и не обращается в нуль на отрезке $\Delta, \Psi$ - решение задачи 1 при $n \in \mathbb{N}$. Тогда при подходящей нормировке полиномов $Q_{n}(z ; \widehat{\rho})$ имеем:

(i) $Q_{n}(z ; \widehat{\rho})=\Psi_{n}(z)(1+o(1))$ при $n \rightarrow \infty$ равномерно внутри $D$;

(ii) $Q_{n}(x ; \widehat{\rho})=\Psi_{n}^{+}(x)+\Psi_{n}^{-}(x)+o(1)$ при $n \rightarrow \infty$ равномерно на $\Delta$.

\footnotetext{
${ }^{15}$ Таким условием $\Psi$-функция определяется однозначно с точностью до знака \pm .
} 
Здесь $\Psi_{n}(z)=\Psi_{n}\left(z^{(1)}\right)$, под $\Psi_{n}^{+}(x)$ (соответственно $\left.\Psi_{n}^{-}(x)\right)$ понимаются верхние (соответственно нижние) предельные значения $\Psi_{n}$ на $\Delta$; величина $o(1)=$ $o\left(\delta^{n}\right)$, где $\delta \in(0,1)$. Из п. (ii) задачи 1 вытекает, что функция $\Psi$, являющаяся решением этой задачи, имеет в бесконечно удаленной точке $\mathbf{z}=\infty^{(1)}$ полюс $n$-го порядка. Поэтому в силу п. (i) теоремы 2 для всех достаточно больших $n$ степень полинома $Q_{n}(z ; \widehat{\rho})$ равна $n$, т. е. все такие индексы нормальны.

Отметим, что для $\rho(x)=(1+x)^{\alpha}(1-x)^{\beta}, x \in \Delta, \alpha, \beta>-1$, имеем:

$$
D(z ; \rho)=\left(\frac{(z+1)^{\alpha}(z-1)^{\beta}}{\Phi(z)^{\alpha+\beta}}\right)^{1 / 2}, \quad z \in D=\overline{\mathbb{C}} \backslash \Delta .
$$

В предположении, что $d \sigma(x)=\left(1-x^{2}\right)^{-1 / 2} \rho(x) d x$, где $\rho$ - комплексная голоморфная функция на $[-1,1], \rho(x) \neq 0$, и

$$
\widehat{\rho}(z):=\int_{-1}^{1} \frac{\rho(x)}{z-x} \frac{d x}{\sqrt{1-x^{2}}}, \quad z \notin \Delta,
$$

в [96] получен следующий результат.

ТЕОРема 3. В классе функций вида $f=\widehat{\rho}+r$, где комплексная функиия $\rho$ голоморфна и не обращается в нуль на отрезке $\Delta$, а все полюсы комплексной рациональной функиии $r$ лежат в области $D$ и $r(\infty)=0$, справедлива импликация

$$
\begin{aligned}
\left(f-f_{n}\right)(z) & =\frac{A}{z^{2 n+1}}+\cdots \\
& \Downarrow \\
\left(f-f_{n}\right)(z) & =\frac{1}{\left(z^{2}-1\right)^{1 / 2} \Psi_{n}(z)^{2}}(1+o(1)), \quad n \rightarrow \infty,
\end{aligned}
$$

равномерно внутри $D^{\prime}$, где $f_{n}, n \in \mathbb{N}$, - рачиональная функиия порядка не выше $n, o(1)=o\left(\delta^{n}\right), \delta<1$, функция $\Psi_{n}$ задана формулой (38).

\section{2. Сходимость аппроксимаций Паде для гиперэллиптических функций}

2.1. В середине 1970-х годов Дж. Наттолл в серии работ [33], [35], [36] (см. также [40], [97]-[99]) получил другой комплексный аналог теоремы Маркова - для общих гиперэллиптических функций. Вместо нескольких отрезков на вещественной прямой он рассмотрел ситуацию, когда носитель меры состоит из нескольких попарно непересекающихся дуг, расположенных в комплексной плоскости. Эти дуги возникают как линии уровня некоторого абелева интеграла третьего рода с чисто мнимыми периодами, заданного на двулистной гиперэллиптической римановой поверхности (ср. [148]). Сама мера предполагалась абсолютно непрерывной относительно меры Лебега (на дугах), а соответствующая весовая функция - достаточно гладкой, а именно такой, чтобы можно было применить классический аппроксимационный метод Бернштейна-Сегё. Здесь мы изложим этот результат Наттолла для случая, когда весовая функция $\rho$ 
голоморфна и отлична от нуля на этих дугах. При этом мы будем использовать другой, более современный подход, впервые предложенный в 1990 г. самим Дж. Наттоллом [149] для случая одного отрезка и основанный на исследовании так называемого сингулярного интегрального уравнения Наттолла, заданного на гиперэллиптической римановой поверхности (см. [149], а также [96]-[98]).

Пусть $H(z)=\prod_{j=1}^{2 p}\left(z-e_{j}\right)-$ полином четной степени с простыми нулями $e_{1}, \ldots, e_{2 p}, \mathfrak{R}$ - двулистная гиперэллиптическая риманова поверхность рода $g=p-1$, заданная уравнением $w^{2}=H(z)$. Каждой точке на $\mathfrak{R}$ соответствует пара $\mathbf{z}=(z, w)$, где $w= \pm \sqrt{H(z)}$. Обозначим через $d G(\mathbf{z})=d G\left(\mathbf{z} ; \infty^{(1)}, \infty^{(2)}\right)$ абелев дифференциал третьего рода на $\mathfrak{R}$ с чисто мнимыми периодами, имеющий в точках $\mathbf{z}=\infty^{(1)}, \infty^{(2)}$ простые полюсы с вычетами $\pm 1 ;$ указанными условиями такой дифференциал определен однозначно (см. [103], [150], [151]). Функция $u(\mathbf{z})=\operatorname{Re} \int_{e_{1}}^{\mathbf{z}} d G(\boldsymbol{\zeta})$ - однозначная гармоническая функция на $\mathfrak{R}$. Линия уровня $\Gamma=\{\mathbf{z} \in \mathfrak{R}: u(\mathbf{z})=0\}$ содержит все точки ветвления и делит поверхность $\mathfrak{R}$ на две подобласти: $D^{(1)}$, где $u(\mathbf{z})>0$, и $D^{(2)}$, где $u(\mathbf{z})<0$. Область $D^{(1)}$ будем отождествлять с ее проекцией $D=\operatorname{proj} D^{(1)}$ на комплексную плоскость $\overline{\mathbb{C}}$. Функция $u(z)=u\left(z^{(1)}\right)$ является функцией Грина с особенностью в бесконечно удаленной точке для области $D$. Будем считать, что точки $e_{1}, \ldots, e_{2 p}$ находятся в общем положении, так что граница $S=\partial D=\operatorname{proj} \Gamma$ coстоит в точности из $p$ непересекающихся компонент $S_{j}$, попарно соединяющих точки ветвления $e_{2 j-1}, e_{2 j}, j=1, \ldots, p$ (занумерованные надлежащим образом). На каждой дуге $S_{j}$ определена ориентация "от $e_{2 j-1}$ к $e_{2 j}$ " и тем самым стандартным образом определены "верхний" $S_{j}^{+}$и "нижний" $S_{j}^{-}$берег и соответствующие предельные значения корня $w^{ \pm}(\zeta)$ при $\zeta \in S$. Другие стандартные сведения о гиперэллиптических римановых поверхностях, которые нам здесь понадобятся, приведены в [103], [150], [151].

Пусть комплексная функция $\rho$ (кусочно) голоморфна на $S$ и не имеет на $S$ нулей (тем самым, $\rho$ голоморфна и $\rho(\zeta) \neq 0$ в некоторой окрестности $S$ ). Следуя Наттоллу (см. [33], [35], [36], [40], [149]), положим

$$
f(z)=\frac{1}{2 \pi i} \int_{S} \frac{\rho(\zeta)}{\zeta-z} \frac{d \zeta}{w^{+}(\zeta)}, \quad z \in D .
$$

В дальнейшем класс функций вида (43) будем обозначать через $\mathscr{N}$.

2.2. Зафиксируем произвольное $n \in \mathbb{N}$ и рассмотрим на римановой поверхности $\mathfrak{R}$ следующую краевую задачу Римана (ср. с задачей 1$)$.

ЗАДАчА 2. При фиксированном $n \in \mathbb{N}, n \geqslant g$, найти функцию $\Psi=\Psi_{n}$ такую, что:

(i) $\Psi$ (кусочно) мероморфна на $\mathfrak{R} \backslash \boldsymbol{\Gamma}=D^{(1)} \sqcup D^{(2)}$;

(ii) дивизор $(\Psi)=(n-g) \infty^{(2)}+\mathbf{z}_{1}+\cdots+\mathbf{z}_{g}-n \infty^{(1)}$;

(iii) на $\boldsymbol{\Gamma}$ выполнено краевое условие: $\rho(\zeta) \Psi^{(1)}(\boldsymbol{\zeta})=\Psi^{(2)}(\boldsymbol{\zeta}), \boldsymbol{\zeta} \in \boldsymbol{\Gamma}$.

В п. (ii) точки $\mathbf{z}_{j}$ - "свободные" нули $\Psi$-функции - зависят от $n$, под $\Psi^{(1)}(\boldsymbol{\zeta})$ в п. (iii) понимаются предельные значения функции $\Psi(\mathbf{z})$ при $D^{(1)} \ni \mathbf{z} \rightarrow \boldsymbol{\zeta} \in \mathbf{\Gamma}$, аналогичный смысл придается и $\Psi^{(2)}(\boldsymbol{\zeta})$. 
Функция $\Psi$, решающая задачу 2 , всегда существует. При этом так как род $g$ поверхности $\mathfrak{R}$ положителен, то нули и полюсы $\Psi$ на $\mathfrak{R}$ оказываются связанными определенными соотношениями, аналогичными соотношениям Абеля для мероморфной функции на $\mathfrak{R}$, а дивизор $d=d(n)=\mathbf{z}_{1}+\cdots+\mathbf{z}_{g}$ является решением проблемы обращения Якоби (напомним, что эта проблема всегда разрешима, но, может быть, не единственным образом). Если индекс $n$ такой, что дивизор $d$ неспециальный, то $\Psi$-функция, решающая задачу (2), единственна с точностью до нормировки и имеет в бесконечно удаленной точке $\mathbf{z}=\infty^{(1)}$ полюс в точности $n$-го порядка. При этом оказывается [98], что равномерно на компактных подмножествах $K$ области $D=\overline{\mathbb{C}} \backslash S$ справедливы асимптотические формулы

$$
Q_{n}(z)=\Psi\left(z^{(1)}\right)(1+o(1)), \quad R_{n}(z)=\frac{\Psi\left(z^{(2)}\right)}{\sqrt{H(z)}}(1+o(1)), \quad n \rightarrow \infty
$$

здесь $z \in K \backslash e_{n}$, сар $e_{n} \rightarrow 0, o(1)=o\left(\delta^{n}\right), n \rightarrow \infty, \delta \in(0,1)$, полиномы $Q_{n}$ нормированы так: старший коэфбициент $Q_{n}$ равен старшему (т. е. при $z^{n}$ ) коэффициенту $\Psi$-функции. Такой нормировки $Q_{n}$ мы будем придерживаться всюду в дальнейшем.

Отметим, что при $p=1$ (т. е. для $g=0$ ) и выборе $e_{1}=-1, e_{2}=1$ имеем: $S=$ $[-1,1], \Psi_{n}(z)=D(z ; \rho) \Phi(z)^{n}$, где $D(z ; \rho)-$ функция Сегё, $\Phi(z)=z+\sqrt{z^{2}-1}$, $|\Phi(z)|>1$ вне $S$ (см. [96]).

Нетрудно увидеть, что для любого $z \in \mathbb{C} \backslash S$ выполняется соотношение $\Psi\left(z^{(1)}\right) \Psi\left(z^{(2)}\right) \equiv \mathrm{const} \prod_{j=1}^{g}\left(z-z_{j}\right)$, где const $\neq 0$. В дальнейшем мы будем придерживаться следующей нормировки ${ }^{16} \Psi$-функции:

$$
\Psi\left(z^{(1)}\right) \Psi\left(z^{(2)}\right) \equiv \prod_{j=1}^{g}\left(z-z_{j}\right)
$$

Полином $X_{n, g}(z)=\prod_{j=1}^{g}\left(z-z_{j}\right)$ фактически является неизвестным полиномиальным параметром задачи 2. Соответствующий точкам $z_{1}, \ldots, z_{g}$ дивизор $d=\mathbf{z}_{1}+\cdots+\mathbf{z}_{g}$, где $\mathbf{z}_{j}=\mathbf{z}_{j}(n) \in \mathfrak{R}$, определяется из решения следующей проблемы обращения Якоби (см. [98], [99], [152], [153]):

$$
\begin{aligned}
\sum_{j=1}^{g} \Omega_{k}\left(\mathbf{z}_{j}\right) \equiv \frac{i}{\pi} & \int_{S} \log \rho(x) d \Omega_{k}^{+}(x)+n \tau_{k}(\infty) \\
& -\sum_{j=1}^{g}\left(n-g+\frac{1}{2}\right) \omega_{j}(\infty) B_{k j}, \quad k=1, \ldots, g .
\end{aligned}
$$

В (46) $d \Omega_{k}, k=1, \ldots, g,-$ базисные $a$-нормированные абелевы дифференциалы первого рода, $\omega_{j}(\infty)$ - гармонические меры кривых $S_{j},\left\|B_{k j}\right\|$ - матрица Римана, равенство правой и левой частей (46) понимается по модулю периодов

\footnotetext{
${ }^{16}$ Таким условием $\Psi$-функция определяется однозначно с точностью до знака \pm .
} 
дифференциалов $d \Omega_{k}$; наконец,

$$
\omega_{k}(\infty)=-\frac{1}{2 \pi i} \oint_{\mathbf{a}_{k}} d G(\mathbf{z}) \in \mathbb{R}_{+}, \quad \tau_{k}(\infty):=\frac{1}{2 \pi i} \oint_{\mathbf{b}_{k}} d G(\mathbf{z}) \in \mathbb{R}
$$

(подробнее см. [98], [99], [153], [154]). В терминах дивизора $d=\mathbf{z}_{1}+\cdots+\mathbf{z}_{g}$ и комплексной функции Грина $G(\mathbf{z})$ можно найти и явный вид $\Psi$-функции (см. [98], [153], [154]). Непосредственно из (44)-(45) и равенства $\left(f-[n / n]_{f}\right)(z)=$ $R_{n}(z) / Q_{n}(z)$ вытекает следующий результат (ср. раздел 1$)$.

ТЕОРема НАТтОЛЛА (см. [36], [98], [153]). Пусть функиия $f$ принадлежит $\mathscr{N}$, т.е. имеет следующий вид:

$$
f(z)=\frac{1}{2 \pi i} \int_{S} \frac{\rho(\zeta)}{\zeta-z} \frac{d \zeta}{w^{+}(\zeta)}, \quad z \in D
$$

точки $e_{1}, \ldots, e_{2 p}$ находятся "в общем положении" и дуги $S_{j}$ не пересекаются. Тогда для диагональных аппроксимаций Паде $[n / n]_{f}$ функции $f$ имеем:

$$
\left(f-[n / n]_{f}\right)(z)=\frac{\prod_{j=1}^{g}\left(z-z_{j}\right)}{\sqrt{H(z)} \Psi_{n}^{2}(z)}(1+o(1)), \quad z \in K \backslash e_{n}, \quad \text { cap } e_{n} \rightarrow 0, \quad n \rightarrow \infty,
$$

где $K$ - произвольный компакт, принадлежащий области D.

Отметим также, что из (44) и (47) вытекает следующая асимптотическая формула:

$$
Q_{n}(z) R_{n}(z)=\frac{\prod_{j=1}^{g}\left(z-z_{j}\right)}{\sqrt{H(z)}}(1+o(1)), \quad z \in K \backslash e_{n}, \quad \operatorname{cap} e_{n} \rightarrow 0, \quad n \rightarrow \infty
$$

(ср. [148], [153; теорема 1, формула (27)], [154; теорема 1, формула (23)]).

Из теоремы Наттолла вытекает следующий результат (ср. (42)).

Теорема 4. В классе функций $f \in \mathscr{N}$ справедлива импликация

$$
\begin{aligned}
\left(f-f_{n}\right)(z) & =\frac{A}{z^{2 n+1}}+\cdots \\
\Downarrow & \\
\left(f-f_{n}\right)(z) & =\frac{\prod_{j=1}^{g}\left(z-z_{j}\right)}{\sqrt{H(z)} \Psi_{n}^{2}(z)}(1+o(1)), \quad n \rightarrow \infty,
\end{aligned}
$$

равномерно по $z \in K \backslash e_{n}, K \subset D$, сар $e_{n} \rightarrow 0$, где $f_{n}, n \in \mathbb{N},-$ рачиональная функиия порядка не выше $n, o(1)=o\left(\delta^{n}\right)$.

ЗАмечАниЕ 2.1. Для случая $g \geqslant 1$ краевая задача 1 впервые сформулирована в работе С.П. Суетина [98] (см. также [152], [153]). Асимптотические формулы (44), непосредственно из которых вытекает теорема Наттолла, также получены в [98]; формула (48) получена в [153]. 
ЗАмечАние 2.2. Случай, когда дуги $S_{j}$ - это отрезки $\Delta_{j}$ на вещественной прямой, впервые рассматривался Н.И. Ахиезером [155], [156] (см. также [40], [148], [157]). Им же в [158] впервые была явно указана связь между нулями полиномов, расположенных в лакунах между отрезками, и проблемой обращения Якоби.

ЗАмечАниЕ 2.3. В работе [99] показано, что динамика точек $z_{k}(n), k=$ $1,2, \ldots, g$, в формуле (48) при $n \rightarrow \infty$ подчиняется системе дифференциальных уравнений Дубровина:

$$
\dot{z}_{k}=-\frac{2 w\left(\mathbf{z}_{k}\right)}{\prod_{j \neq k}\left(z_{k}-z_{j}\right)} \int_{e_{2 g+2}}^{\infty} \frac{\prod_{j \neq k}\left(x-z_{j}\right)}{w(x)} d x, \quad k=1, \ldots, g,
$$

где $\dot{z}_{k}=d z_{k} / d t, e_{2 g+2}$ - крайняя правая точка носителя меры $\sigma$ (предполагается, что концевые точки отрезков носителя меры занумерованы в порядке возрастания их величин, а путь интегрирования в (50) - часть вещественной оси $\left[e_{2 g+2},+\infty\right)$; подробнее по поводу обозначений см. [99]). Точнее, в представления для решений $z_{k}=z_{k}(t)$ системы (50) вместо непрерывного вещественного параметра $t \in \mathbb{R}_{+}$нужно подставить дискретный параметр $n \in \mathbb{N}$ (см. [99], [153]). При этом для $y$-координат соответствующего дивизора $d(n)=$ $\mathbf{z}_{1}(n)+\cdots+\mathbf{z}_{g}(n) \in S^{g} \mathfrak{R}$ справедлива следующая явная формула (см. [153; формула (В.27)]):

$$
y_{k}=\left(n-\frac{g}{2}\right) \omega_{k}(\infty)+\frac{1}{\pi} \int_{E} \log |\rho(x)| \frac{\partial \omega_{k}}{\partial n^{+}}(x) d x \quad(\bmod 1) .
$$

\section{3. Теорема Шталя}

3.1. Пусть $\Sigma=\left\{a_{1}, \ldots, a_{m}\right\} \subset \mathbb{C}-$ конечное множество. Через $\mathscr{A}(\overline{\mathbb{C}} \backslash \Sigma)$ обозначим класс всех таких функций $f$, что $f$ голоморфна в точке $z=\infty$ и допускает аналитическое продолжение вдоль любого пути в $\overline{\mathbb{C}} \backslash \Sigma$; при этом предполагается, что $f \notin \mathscr{H}(\overline{\mathbb{C}} \backslash \Sigma)$, т. е. соответствующая полная аналитическая функция многозначна. Пусть $\mathscr{G}_{f}-$ класс всех областей $G \subset \overline{\mathbb{C}}$, содержащих бесконечно удаленную точку и таких, что функция $f$ продолжается в область $G$ как голоморфная (т. е. однозначная аналитическая) функция: $\mathscr{G}_{f}=\{G \subset \overline{\mathbb{C}}, G \ni \infty, f \in \mathscr{H}(G)\}$. Через $\mathscr{K}_{f}$ обозначим класс всех компактов $K$ таких, что $K=\mathbb{C} \backslash G$ для некоторой области $G \in \mathscr{G}_{f}$. Области $G \in \mathscr{G}_{f}$ и соответствующие им компакты $K \in \mathscr{K}_{f}$ будем называть допустимыми (для функции $f$ ).

Пусть $f \in \mathscr{A}(\overline{\mathbb{C}} \backslash \Sigma)$ и $\left\{[n / n]_{f}\right\}$ - последовательность ее диагональных аппроксимаций Паде (в точке $z=\infty$ ). Тогда в классе $\mathscr{G}_{f}$ существует единственная максимальная область $D=D_{f} \in \mathscr{G}_{f} u[n / n]_{f} \rightarrow f$ nри $n \rightarrow \infty$ внутри области $D$ (Г. Шталь, см. [63]-[67], а также [70]). Здесь максимальность допустимой области $D$ понимается в том смысле, что дополнение $F=\overline{\mathbb{C}} \backslash D$ к ней минимально, точнее $F \in \mathscr{K}_{f}$ имеет наименьшую емкость в классе всех допустимых компактов $\mathscr{K}_{f}$. 
Например, для функции $f(z)=\frac{1}{\sqrt{z^{2}-1}}$ компакт Шталя $F$ есть $[-1,1]$, а область Шталя $D$ есть $\overline{\mathbb{C}} \backslash[-1,1]$; для функции $f(z)=\frac{1}{\sqrt[3]{\left(z-a_{1}\right)\left(z-a_{2}\right)\left(z-a_{3}\right)}}$, где три точки $a_{1}, a_{2}, a_{3}$ не лежат на одной прямой, компакт Шталя $F$ совпадает с компактом Чеботарева $E\left(a_{1}, a_{2}, a_{3}\right)$ для трех точек, соответственно область Шталя $D$ совпадает с $\overline{\mathbb{C}} \backslash E\left(a_{1}, a_{2}, a_{3}\right)$ (см. [159], [160]).

3.2. Перейдем теперь к точной формулировке теоремы Шталя.

Пусть функция $f$ задана сходящимся в точке $z=\infty$ рядом Лорана

$$
f(z)=\sum_{k=0}^{\infty} \frac{c_{k}}{z^{k+1}}
$$

и принадлежит классу $\mathscr{A}(\overline{\mathbb{C}} \backslash \Sigma)$. Обозначим через $[n / n]_{f}$ диагональную аппроксимацию Паде функции $f:[n / n]_{f}=P_{n} / Q_{n}$, где $\operatorname{deg} P_{n}, \operatorname{deg} Q_{n} \leqslant n, Q_{n} \not \equiv 0$ и выполняется соотношение

$$
\left(Q_{n} f-P_{n}\right)(z)=O\left(\frac{1}{z^{n+1}}\right), \quad z \rightarrow \infty .
$$

Для произвольного компакта $K \subset \overline{\mathbb{C}}$, не разбивающего плоскость, через $D_{K}$ обозначим связную компоненту дополнения к $K$, содержащую точку $z=\infty$. Пусть $\mathscr{K}_{f}$ - семейство компактов $K \subset \overline{\mathbb{C}}$ таких, что область $D(K)$ регулярна относительно решения задачи Дирихле и $f$ допускает голоморфное (однозначное аналитическое) продолжение из окрестности точки $z=\infty$ в область $D(K)$ : $f \in \mathscr{H}\left(D_{K}\right)$. Компакты $K \in \mathscr{K}_{f}$ будем называть допустимыми для функции $f \in \mathscr{A}(\overline{\mathbb{C}} \backslash \Sigma)$.

Обозначим $\mu(Q)$ меру, ассоциированную с произвольным полиномом $Q$ :

$$
\mu(Q)=\sum_{\zeta: Q(\zeta)=0} \delta_{\zeta}
$$

где $\delta_{\zeta}$ - мера Дирака с носителем в точке $\zeta$, сумма берется по всем точкам $\zeta-$ нулям полинома $Q$.

Следующий результат о сходимости диагональных аппроксимаций Паде принадлежит Г. Шталю [63]-[67].

ТЕОрема ШтАЛЯ. Пусть $f \in \mathscr{A}(\mathbb{C} \backslash \Sigma)$. Тогда:

1) существует единственный компакт $F=F(f)$ такой, что $F \in \mathscr{K}_{f} u$

$$
\operatorname{cap} F=\min _{K \in \mathscr{K}_{f}} \operatorname{cap} K
$$

компакт F состоит из конечного числа кусочно аналитических дуг, не разбивает плоскость и является замыканием критических траекторий некоторого квадратичного дифференииала;

2) для нулей $Q_{n}$ (знаменателей рачиональных функиий $[n / n]_{f}$ ) справедливо предельное соотношение:

$$
\frac{1}{n} \mu\left(Q_{n}\right) \rightarrow \lambda_{F}, \quad n \rightarrow \infty
$$


где $\lambda_{F}-($ единичная) равновесная мера компакта $F$,

$$
d \lambda_{F}(z)=\frac{1}{i \pi} \sqrt{\frac{B_{m-2}}{A_{m}}(z)} d z, \quad z \in F
$$

при надлежащем выборе ветви квадратного корня, $A_{m}(z)=\prod_{j=1}^{m}\left(z-a_{j}\right)$, $B_{m-2}-$ некоторый полином степени $\leqslant m-2$, сходимость мер понимается в слабой топологии;

3) последователъность $[n / n]_{f}$ сходится по емкости внутри (на компактных подмножествах) области $D=\overline{\mathbb{C}} \backslash F \kappa$ функции $f$ :

$$
\lim _{n \rightarrow \infty}\left|f(z)-[n / n]_{f}(z)\right|^{1 / n}=e^{-2 g_{F}(z, \infty)}<1,
$$

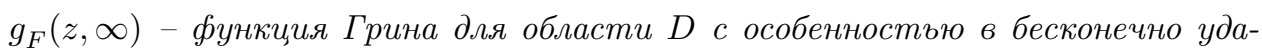
ленной точке.

Отметим, что область Шталя $D$ является областью максимальной сходимости аппроксимаций Паде функции $f$; при этом скачок $\Delta f(\zeta)=\left(f^{+}-f^{-}\right)(\zeta)$, $\zeta \in \ell$, функции $f$ на любой открытой дуге $\ell \subset F=\partial D$ отличен от тождественного нуля: $\Delta f(\zeta) \not \equiv 0, \zeta \in \ell$.

Соотношение (56) означает следующее: для любого компакта $K \Subset D_{f}$ и любого числа $\varepsilon>0$ найдутся номер $n_{0}=n_{0}(K, \varepsilon)$ и число $\delta=\delta(K, \varepsilon)>0$ такие, что при всех $n \geqslant n_{0}$ справедливо соотношение

$$
\left(e^{-2 g(z)-\varepsilon}\right)^{n} \leqslant\left|\left(f-[n / n]_{f}\right)(z)\right| \leqslant\left(e^{-2 g(z)+\varepsilon}\right)^{n}, \quad z \in K \backslash e_{n}, \quad \operatorname{cap} e_{n}<\delta .
$$

Здесь $g(z)=g_{F}(z, \infty)$.

Из (57) уже легко следует, что каждый полюс а функиии $f$ в области $D$ кратности $\nu(a) \geqslant 1$ притягивает при $n \rightarrow \infty$ по менъшей мере $\nu(a)$ полюсов рациональной функиии $[n / n]_{f}$.

3.3. Метод Шталя основан на том, что экстремальный компакт $F$ (см. (54)) является совокупностью замыканий критических траекторий некоторого квадратичного дифференциала, состоит из конечного числа аналитических дуг, не разбивает плоскость и вполне характеризуется следующим свойством симметрии (или $S$-свойством):

$$
\frac{\partial g_{F}(\zeta, \infty)}{\partial n_{+}}=\frac{\partial g_{F}(\zeta, \infty)}{\partial n_{-}}, \quad \zeta \in F^{0}
$$

$F^{0}$ - совокупность открытых аналитических дуг, составляющих $F, \partial n_{ \pm}-$производные по нормали с противоположных сторон $F^{0}$. Свойство неограниченной продолжаемости функции $f$ в $\overline{\mathbb{C}} \backslash \Sigma$ не существенно; важен лишь тот факт, что $f$ имеет "правильный" скачок $\Delta f(\zeta)$ на некотором компакте $F$, удовлетворяющем условию (58). В силу равенства $g_{F}(z, \infty)=\gamma_{F}-V^{\lambda}(z)$, где $\lambda=\lambda_{F}$ - равновесная мера для $F, \gamma_{F}$ - постоянная Робена, соотношение (58) эквивалентно соотношению

$$
\frac{\partial V^{\lambda}(\zeta, \infty)}{\partial n_{+}}=\frac{\partial V^{\lambda}(\zeta, \infty)}{\partial n_{-}}, \quad \zeta \in F^{0}
$$


Компакты, обладающие тем или иным $S$-свойством, часто возникают в различных задачах теории аппроксимаций и геометрической теории функций. В частности, свойством симметрии обладают экстремальные кривые целого ряда классических экстремальных задач геометрической теории функций; в этой теории экстремальные кривые обычно описываются как траектории некоторого квадратичного дифференциала (см. [159]-[162]).

Теорема Шталя доказывается по следующей схеме (см. [63]-[67]). Вначале на основе достаточно простых геометрических соображений устанавливается, что существует допустимый компакт $F \in \mathscr{K}_{f}$ такой, что

$$
\operatorname{cap} F=\min _{K \in \mathscr{K}_{f}} \operatorname{cap} K \text {. }
$$

Затем выясняется структура $F$, а именно доказывается, что $F$ является замыканием критических траекторий некоторого квадратичного дифференциала. Отсюда уже вытекает $S$-свойство (58). Непосредственно на основе этого $S$-свойства устанавливается предельное соотношение (55) для знаменателей диагональных аппроксимаций Паде $[n / n]_{f}$. Соотношение (55) влечет сходимость по емкости последовательности $\left\{[n / n]_{f}\right\}$ к функции $f$ внутри (на компактных подмножествах) области $D$. Единственность компакта $F$, удовлетворяющего условию (59), вытекает из сходимости (55).

Соотношение (55) эквивалентно соотношению

$$
\left|Q_{n}(z)\right|^{1 / n} \stackrel{\text { cap }}{\longrightarrow} C e^{g_{F}(z, \infty)}=e^{-V^{\lambda}(z)}, \quad z \in D, \quad C=\frac{1}{\operatorname{cap} F}=e^{\gamma_{F}},
$$

где старший коэффициент полинома $Q_{n}$ равен единице.

Отметим, что в [163] (см. также [129], [130]) предложен другой подход для доказательства того, что экстремальный компакт $F$ обладает $S$-свойством. Этот подход основан на вариационном методе и связи емкости компакта с логарифмической энергией его равновесной меры. Тем самым, вариация компакта порождает вариацию соответствующего функционала энергии. Выбором подходящей вариации удается получить "необходимое" условие экстремальности компакта $F$, непосредственно из которого и вытекает требуемое $S$-свойство. Такой подход позволил установить $S$-свойство стационарных компактов и для других функционалов энергии, естественным образом возникающих в различных задачах теории аппроксимаций и математической физики (см. [80], [127]-[130], [136]).

\section{4. Равновесная мера и распределение нулей экстремальных многочленов}

4.1. А. А. Гончаром и Е. А. Рахмановым было замечено [26], [47] (см. также [12], [52], [46], [50]-[57], [164]), что решение ряда задач теории приближений может быть сведено к задаче о равновесном распределении заряда на "проводнике" при наличии тех или иных "внешних полей" (см. ниже теорему 5). Задачи такого типа возникают, например, в теории сходимости аппроксимаций Паде и Эрмита-Паде (см. прежде всего [26], [46], [47], [49]), где ключевую роль играют асимптотические свойства (неэрмитово) ортогональных многочленов. 
Вопросы, связанные с предельным поведением экстремальных (в том или ином смысле) полиномов, важны как в самом анализе, так и в приложениях. В последние два десятилетия эти вопросы оказались тесно связанными с задачами об асимптотических свойствах случайных матриц. Поэтому большой интерес представляют общие методы исследования асимптотики таких полиномов.

Здесь приводится достаточно общая теорема в этом направлении, принадлежащая А. А. Гончару и Е. А. Рахманову: теорема 6 характеризует предельное распределение нулей многочленов $P_{n}, n=1,2, \ldots$, определяемых одним (при каждом $n$ ) экстремальным соотношением (см. ниже (80)) с переменной (зависящей от $n$ ) весовой функцией. Наибольший интерес представляет случай, когда многочлены $P_{n}(x)=x^{n}+\cdots, n=1,2, \ldots$, определяются соотношениями ортогональности вида

$$
\int_{a}^{b} P_{n}(x) x^{j} \varphi_{n}(x) d x=0, \quad j=0,1, \ldots, n-1,
$$

где $\varphi_{n}$ - заданная последовательность весовых функций на (ограниченном или неограниченном) промежутке $\langle a, b\rangle$ вещественной прямой $\mathbb{R}$. Многочлен $P_{n}$ минимизирует норму

$$
\left(\int_{a}^{b}\left|P_{n}(x)\right|^{2} \varphi_{n}(x) d x\right)^{1 / 2}
$$

в классе всех многочленов степени $n$ с единичным старшим коэффициентом; распределение нулей таких многочленов, как показано ниже, зависит от асимптотического поведения функций $\varphi_{n}^{1 / n}$ при $n \rightarrow \infty$. Аналогичные результаты справедливы для многочленов, удовлетворяющих системам экстремальных соотношений (в частности, системам соотношений ортогональности); теоретико-потенциальный подход к задачам, связанным с предельным распределением нулей таких многочленов, был впервые предложен в статье [26].

В пп. 4.2, 4.3 ниже рассматривается задача равновесия при наличии "внешнего поля" и соответствующая ей экстремальная задача для потенциалов положительных зарядов (мер); здесь рассматривается случай, когда "проводник" промежуток вещественной прямой $\mathbb{R}$. Теорема о предельном переходе от дискретных экстремальных задач к соответствующей экстремальной задаче для потенциалов мер сформулирована в п. 4.2. В пп. 4.2.1, 4.2.2 выделены некоторые частные случаи рассматриваемой задачи; относящиеся к ним следствия теоремы обобщают и дополняют результаты известных ранее работ, относящихся к распределению нулей соответствующих экстремальных многочленов.

4.2. В дальнейшем мера - это положительная борелевская мера в комплексной плоскости $\mathbb{C}$, принимающая конечные значения на компактах в $\mathbb{C}$; $S_{\mu}$ - носитель меры $\mu,|\mu|=\mu(\mathbb{C}), V^{\mu}$ - ее логарифмический потенциал:

$$
V^{\mu}(z)=\int \log \frac{1}{|z-t|} d \mu(t), \quad z \in \mathbb{C} .
$$

Потенциалы рассматриваются здесь только для мер $\mu$, удовлетворяющих условию

$$
\int_{|t| \geqslant 1} \log \frac{1}{|t|} d \mu(t)>-\infty
$$


для таких мер $V^{\mu}(z) \in(-\infty,+\infty], z \in \mathbb{C}$. Отметим, что все используемые ниже понятия и факты теории потенциала содержатся в монографии [165] (см. также [51]).

Потенциал $V^{\mu}(z)$ - супергармоническая (в частности, полунепрерывная снизу) функция в $\mathbb{C}$. Множество $\left\{z: V^{\mu}(z)=+\infty\right\}$ имеет нулевую емкость. В каждой точке $z_{0} \in \mathbb{C}$ потенциал $V^{\mu}$ непрерывен в тонкой топологии: существует множество $e_{0}\left(z_{0}\right)$, разреженное в точке $z_{0}$ и такое, что $V^{\mu}(z) \rightarrow V^{\mu}\left(z_{0}\right)$ при $z \rightarrow z_{0}, z \notin e_{0}\left(z_{0}\right)$.

Отметим вытекающие отсюда свойства потенциалов мер на вещественной прямой $\mathbb{R}$. Функция $V^{\mu}(x), x \in \mathbb{R}$, полунепрерывна снизу на $\mathbb{R}$. Для борелевских множеств $e \subset \mathbb{R}$ имеет место неравенство $|e| \leqslant 4$ сар $e$, где $|\cdot|-$ мера Лебега на прямой, сар $(\cdot)$ - логарифмическая емкость. В частности, множества нулевой емкости имеют нулевую меру и $V^{\mu}(x)<+\infty$ почти всюду на $\mathbb{R}$ (относительно меры Лебега). Разреженное в точке $x_{0}$ множество $e_{0} \subset \mathbb{R}$ имеет нулевую плотность в этой точке; поэтому функция $V^{\mu}(x)$ аппроксимативно непрерывна в каждой точке $x_{0} \in \mathbb{R}$.

Фиксируем замкнутый промежуток $F \subset \mathbb{R}$. Через $\mathscr{F}=\mathscr{F}(F)$ обозначим класс всех функций $f: F \rightarrow(-\infty,+\infty]$, удовлетворяющих условиям:

a) $f$ полунепрерывна снизу и слабо аппроксимативно непрерывна на $F$, т. е. для любой точки $x_{0} \in F$ имеем

$$
\varliminf_{x \rightarrow x_{0}, x \in F} f(x)=\lim _{x \rightarrow x_{0}, x \in e\left(x_{0}\right)} f(x)=f\left(x_{0}\right)
$$

где $e\left(x_{0}\right)$ - некоторое множество (зависящее от $f$ ), имеющее положительную плотность в точке $x_{0}$;

b) выполнено соотношение

$$
\varliminf_{|x| \rightarrow \infty, x \in F} \frac{f(x)}{\log |x|}>1 .
$$

Из а) следует, что для $f \in \mathscr{F}$ множество $f(x)<+\infty$ имеет положительную меру. Для компактного случая $(F=[a, b]$ - отрезок) сужения на $F$ потенциалов мер принадлежат классу $\mathscr{F}$; условие а) получено существенным ослаблением отмеченных выше свойств непрерывности потенциалов мер. Более того, в этом случае функции вида $V^{\mu}+f(\mu-$ мера, $f \in \mathscr{F})$ также принадлежат классу $\mathscr{F}$. Отметим, что многие важные для приложений функции $f$ (например, кусочно непрерывные функции на отрезке $F$ при наличии разрывов) принадлежат классу $\mathscr{F}$, но не являются потенциалами мер. Функции $f \in \mathscr{F}$ играют роль "внешних полей" в задаче о распределении положительного заряда (меры) на "проводнике" $F$.

Множество всех единичных мер $\mu$ на $F\left(S_{\mu} \subset F\right)$, удовлетворяющих условию (64), обозначим через $M(F)$.

Положим

$$
w(\mu, f)=\min _{x \in F}\left(V^{\mu}+f\right)(x)
$$

здесь $\mu$-мера, $f \in \mathscr{F}$. 
Теорема 5 (Гончар, Рахманов [47]). Пусть $f$ - произвольная функция

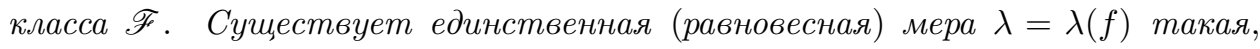
что

$$
\left(V^{\lambda}+f\right)(x) \equiv w(\lambda, f), \quad x \in S_{\lambda} .
$$

Мера $\lambda=\lambda(f)$ является единственным решением следующей экстремальной задачи:

$$
w(\lambda, f)=\max _{|\mu|=1} w(\mu, f) .
$$

Соотношение равновесия (68) может быть записано в виде

$$
\begin{aligned}
\left(V^{\lambda}+f\right)(x) & =w, \quad x \in S_{\lambda} \\
& \geqslant w, \quad x \in F \backslash S_{\lambda},
\end{aligned}
$$

где $w$ - некоторая константа; первое утверждение теоремы 5 означает, что свойство (70) однозначно определяет меру $\lambda \in M(F)$ (и, тем самым, константу $w$ ). Второе утверждение теоремы 5 можно записать в виде следующей импликации:

$$
w(\mu, f) \geqslant w(\lambda, f), \quad|\mu|=1 \quad \Longrightarrow \quad \mu=\lambda .
$$

Мера $\lambda=\lambda(f)$ называется равновесной мерой, соответствующей функции $f \in \mathscr{F}$, константа $w=w(f)=w(\lambda(f), f)$ - экстремальной константой. Непосредственно из условия равновесия (70) вытекает, что потенциал $V^{\lambda}$ непрерывен на $S_{\lambda}$ и, тем самым, непрерывен в $\mathbb{C}$.

Приведем доказательство теоремы 5. Отметим, что оно проводится стандартными методами теории потенциала (ср. [165; гл. II, §1, формула (2.1.2)] для случая $f \equiv 0$, а также [49; $§ 3$, п. 2 , лемма 6$]$ и $[58 ; \S 6.6]$ в общем случае). В основе доказательства лежит следующий "вариационный принцип" : для произвольного вещественного параметра $\varepsilon \in(0,1)$ мера $\varepsilon \lambda+(1-\varepsilon) \mu \in M(\mathbb{R})$ имеет большую энергию, чем равновесная мера $\lambda$.

ДокАзАтЕЛьство. Пусть сначала $F$ - отрезок,

$$
I(\mu):=\iint_{F \times F} \log \frac{1}{|x-y|} d \mu(x) d \mu(y), \quad J(\mu, f):=I(\mu)+2 \int_{F} f(x) d \mu(x)
$$

- (удвоенные) собственная энергия меры $\mu$ и полная энергия с учетом взаимодействия с внешним полем $f$. Через $M^{0}(F)$ обозначим множество мер $\mu \in M(F)$, имеющих конечную энергию $I(\mu)$. Из слабой компактности $M(F)$ и принципа понижения [165; гл. I, $\S 3$, теорема 1.3] для $J(\mu, f)$ следует существование минимизирующей меры $\lambda$ :

$$
J(\lambda, f)=\min _{\mu \in M(F)} J(\mu, f) .
$$

Из соотношений (для мер из $M^{0}(F)$ )

$$
\begin{gathered}
J\left(\frac{\mu+\nu}{2}, f\right) \leqslant \frac{1}{2}(J(\mu, f)+J(\nu, f)), \\
I(\mu-\nu)=2 J(\mu, f)+2 J(\nu, f)-4 J\left(\frac{\mu+\nu}{2}, f\right)
\end{gathered}
$$


следует единственность минимизирующей меры $\lambda$. Далее, непосредственно проверяется, что для любого $\varepsilon>0$ и любой меры $\sigma \in M^{0}(F)$ справедливо тождество

$$
J(\varepsilon \sigma+(1-\varepsilon) \lambda, f)-J(\lambda, f)=2 \varepsilon \int\left(V^{\lambda}+f\right)(x) d(\sigma-\lambda)(x)+\varepsilon^{2} I(\sigma-\lambda) .
$$

Отсюда вытекает, что минимизирующая мера $\lambda$ является единственной мерой из $M^{0}(F)$, удовлетворяющей условию

$$
\int\left(V^{\lambda}+f\right)(x) d(\sigma-\lambda)(x)>0 \quad \text { для любой мерь } \sigma \in M^{0}(F) .
$$

В самом деле, из (73) и (74) немедленно следует (75) (при $\varepsilon \rightarrow 0$ ). С другой стороны, в силу позитивности энергии $I(\nu)$ на нейтральных зарядах, имеем $I(\sigma-\lambda) \geqslant 0$ для любой меры $\sigma \in M^{0}(F)$; используя $(74)$ с $\varepsilon=1$, заключаем, что всякая мера $\lambda \in M^{0}(F)$, удовлетворяющая (75), минимизирует интеграл энергии $J(\mu, f)$.

Если мера $\lambda$ удовлетворяет условию (75), то для нее справедливы соотношения равновесия (70) с

$$
w=\int\left(V^{\lambda}+f\right)(x) d \lambda(x) .
$$

Действительно, если $\left(V^{\lambda}+f\right)(x)<w$ на замкнутом множестве $e \subset F$, сар $e>0$, то существует $\sigma \in M^{0}(e)$, для которой $\int\left(V^{\lambda}+f\right)(x) d \sigma(x)<w$ и, тем самым, нарушено (75). Следовательно, $\left(V^{\lambda}+f\right)(x) \geqslant w$ приблизительно всюду на $F$. Если $\left(V^{\lambda}+f\right)(x)>w$ на непустом множестве $e \subset S(\lambda)$, то из полунепрерывности снизу функции $V^{\lambda}+f$ следует неравенство $\int\left(V^{\lambda}+f\right)(x) d \lambda(x)>w$;

Если $\lambda$ - равновесная мера, то $\left(V^{\lambda}+f\right)(x) \leqslant w$ всюду на $S(\lambda)$; тем самым, $\lambda \in M^{0}(F)$. Поскольку при интегрировании по мерам из $M^{0}(F)$ множества нулевой внутренней емкости не играют роли, получаем (75).

Таким образом, минимизирующая мера $\lambda$ и только эта мера удовлетворяет соотношениям равновесия (70).

Если теперь $F$ - неограниченный промежуток, то для каждого $r>0$ положим $F_{r}=F \cap[-r, r]$ и обозначим через $\lambda_{r}$ равновесную меру, соответствующую отрезку $F_{r}$ и функции $f(x), x \in F_{r}$. С помощью условия b) в определении класса $\mathscr{F}$ (см. (66)) нетрудно доказать, что существует конечное положительное число $R$ такое, что $\lambda_{r}=\lambda_{R}$ при всех $r \geqslant R$. Мера $\lambda_{R}$ является единственным решением задач (70) и (71) для промежутка $F$.

Теорема 5 доказана.

Положим

$$
S^{\lambda}=\left\{x \in \mathbb{R}:\left(V^{\lambda}+f\right)(x)=w(\lambda, f)\right\} .
$$

Тогда условие равновесия (70) может быть представлено в следующей эквивалентной форме:

$$
S_{\lambda} \subseteq S^{\lambda} .
$$


Подчеркнем, что соотношение (77) эквивалентно условию равновесия (70): мера $\lambda$ является единственной мерой из $M(\mathbb{R})$, удовлетворяющей условию $(77)$.

Даже для достаточно простых функций $f$ нахождение равновесной меры $\lambda=\lambda(f)$ и экстремальной константы $w=w(f)$ затрудняется тем, что носитель меры $\lambda$ заранее не известен. Вычисления (основанные на соотношениях $(70),(71))$ упрощаются в тех случаях, когда заранее известно, что носитель равновесной меры - отрезок (или несколько отрезков, см. ниже п. 4.3). Последнее часто удается установить на основе общих свойств функции $f$; это так, например, для выпуклых (вниз) функций $f$. В предположении, что носитель равновесной меры $\lambda$ - отрезок $[\alpha, \beta]$, нахождение меры $\lambda$ и потенциала $V^{\lambda}$ основывается на соотношении равновесия (70); это соотношение приводит, в частности, к хорошо изученному уравнению

$$
\int_{\alpha}^{\beta} \log |t-x| d \lambda(t) \equiv f(x)+\text { const, } \quad x \in[\alpha, \beta],
$$

для определения меры $\lambda, \lambda([\alpha, \beta])=1$. Если при этом какая-либо из точек $\alpha, \beta$ является внутренней точкой промежутка $F$, то из $(70)$ следует, что в этой точке $\left(V^{\lambda}+f\right)^{\prime}(x)=0$ (в предположении, что $f$ дифференцируема в рассматриваемой точке). Это замечание позволяет в ряде случаев получить уравнения для определения точек $\alpha, \beta$. Ограничимся двумя примерами, в которых вычисления доводятся до явных формул; по существу, эти примеры разобраны в [26], [46] (см. также [47]).

4.2.1. Пусть $F=[a, b]$ - отрезок, $\mu$ - фиксированная мера с компактным носителем, принадлежащим $\mathbb{R} \backslash(a, b), f=\left.V^{\mu}\right|_{F}, \lambda=\lambda(f)$ и $w=w(f)$. В этом случае $S_{\lambda}=[\alpha, \beta], a \leqslant \alpha<\beta \leqslant b$. Точки $\alpha, \beta$ могут быть найдены по следующему правилу. Пусть $x_{1}, x_{2} \in[a, b]$; положим

$$
h\left(x_{1}, x_{2}\right)=\frac{1}{1+|\mu|} \int\left|\frac{t-x_{1}}{t-x_{2}}\right|^{1 / 2} d \mu(t) .
$$

Если $h(a, b) \leqslant 1$ и $h(b, a) \leqslant 1$, то $\alpha=a, \beta=b$; если $h(a, b) \leqslant 1, h(b, a)>1$, то $\beta=b$, а $\alpha \in(a, b)$ - решение уравнения $h(b, \alpha)=1$; аналогично, если $h(a, b)>1$, $h(b, a) \leqslant 1$, то $\alpha=a, \beta \in(a, b)$ - решение уравнения $h(a, \beta)=1$; наконец, если $h(a, b)>1$ и $h(b, a)>1$, то $\alpha, \beta(a<\alpha<\beta<b)$ - решение системы уравнений $h(\alpha, \beta)=h(\beta, \alpha)=1$. Во всех случаях существование единственного решения заранее известно.

Формулы для $d \lambda$ и $w$ имеют вид

$$
\begin{gathered}
d \lambda(x)=\frac{|(x-\alpha)(x-\beta)|^{1 / 2}}{\pi} \int \frac{1}{|(t-\alpha)(t-\beta)|^{1 / 2}} \frac{d \mu(t)}{|t-x|} d x, \quad x \in[\alpha, \beta], \\
w=(1+|\mu|) \log \frac{4}{\beta-\alpha}-\int g(t, \infty) d \mu(t),
\end{gathered}
$$

где $g(t, \infty)$ - функция Грина для дополнения к отрезку $[\alpha, \beta]$.

4.2.2. Пусть $F=\mathbb{R}, f(x)=c|x|^{p}$ ( $c, p$ - положительные константы), $\lambda=\lambda(f)$ и $w=w(f)$. Имеем $S_{\lambda}=[-\alpha, \alpha]$, где

$$
\alpha=\left(\frac{A_{p}}{2 c}\right)^{1 / p}, \quad A_{p}=\frac{\sqrt{\pi} \Gamma(p / 2)}{\Gamma((p+1) / 2)}
$$


(здесь $\Gamma$ - гамма-функция Эйлера). Если $c=c_{p}=A_{p} / 2$, то $S_{\lambda}$ - единичный отрезок; в этом случае $d \lambda$ и $w$ находятся по формулам

$$
\begin{gathered}
d \lambda(x)=\frac{p}{\pi} \int_{|x|}^{1} \frac{|x|^{p-1} d t}{t^{p}\left|1-t^{2}\right|^{1 / 2}} d x, \quad x \in[-1,1], \\
w=\log \left(2 e^{1 / p}\right) .
\end{gathered}
$$

Прежде чем формулировать общую теорему о предельном распределении нулей экстремальных многочленов, приведем соответствующий результат для простого частного случая.

Пусть $F=[a, b]$ - отрезок, $s$ - фиксированное положительное число и $\varphi_{n}$, $n=1,2, \ldots,-$ последовательность непрерывных неотрицательных функций на $[a, b]$. Обозначим через $P_{n}(x)=x^{n}+\cdots-$ многочлен, минимизирующий норму

$$
\left(\int_{a}^{b}|P(x)|^{s} \varphi_{n}(x) d x\right)^{1 / s}
$$

в классе всех многочленов $P=P(x)$ степени $n$ с единичным старшим коэффициентом. Ясно, что асимптотическое поведение многочленов $P_{n}, n=1,2, \ldots$, в частности, распределение нулей этих многочленов, зависит от асимптотического поведения функций $\varphi_{n}, n=1,2, \ldots$. Основной качественный вывод из приводимой ниже теоремы 6 состоит в том, что предельное распределение нулей многочленов $P_{n}$ определяется асимптотическим поведением последовательности функций $\varphi_{n}^{1 / n}$. В частности, если последовательность $\varphi_{n}^{1 / n}$ равномерно сходится на отрезке $[a, b] \kappa$ функиии $\varphi \not \equiv 0$ ( $\varphi$ - неотрицательная непрерывная функция на $[a, b])$, то нули многочленов $P_{n}, n=1,2, \ldots$, имеют предельное распределение, которое характеризуется равновесной мерой $\lambda(f), f=\frac{1}{s} \log \frac{1}{\varphi}$ $\left(\log \frac{1}{0}=+\infty\right):$ для любого отрезка $\Delta \subset[a, b]$ справедливо соотношение

$$
\lim _{n \rightarrow \infty} \frac{1}{n} \varkappa_{n}(\Delta)=\int_{\Delta} d \lambda(x), \quad \lambda=\lambda\left(\frac{1}{s} \log \frac{1}{\varphi}\right)
$$

где $\varkappa_{n}(\Delta)$ - число нулей многочлена $P_{n}$ на отрезке $\Delta$.

Перейдем теперь к определениям и обозначениям, которые позволят сформулировать общую теорему.

Пусть $F-\oint$ - ксированный замкнутый промежуток прямой $\mathbb{R}$. Кроме того, фиксируем:

(i) число $s \in(0,+\infty]$;

(ii) меру $\sigma$ на $F$ такую, что $d \sigma(x)=h(x) d x, h \in L_{\text {loc }}^{1}(F)$ почти всюду на $F$ и $h(x) \rightarrow$ const при $|x| \rightarrow \infty, x \in F$;

(iii) последовательность функций $f_{n} \in \mathscr{F}, n=1,2, \ldots$.

Определим при каждом $n=1,2, \ldots$ норму $N_{n}(P)$ на множестве всех многочленов $P=P(x)$ степени не выше $n$ :

$$
N_{n}(P)= \begin{cases}\left(\int_{F}\left|P \varphi_{n}\right|^{s} d \sigma\right)^{1 / s}, & s \in(0,+\infty), \\ \max _{F}\left|P \varphi_{n}\right|, & s=\infty,\end{cases}
$$


где

$$
\varphi_{n}=e^{-n f_{n}} .
$$

Иначе говоря, рассматривая последовательность норм $N_{n}(\cdot)$ вида (82), мы предполагаем, что каждая из функций

$$
f_{n}=\frac{1}{n} \log \frac{1}{\left|\varphi_{n}\right|}
$$

принадлежит классу $\mathscr{F}$.

Пусть $\mathscr{P}_{n}^{*}-$ класс всех многочленов степени $n$ с единичным старшим коэффициентом. Обозначим через $P_{n}$ многочлен из $\mathscr{P}_{n}^{*}$, минимизирующий норму $N_{n}(\cdot)$ в классе $\mathscr{P}_{n}^{*}$ :

$$
N_{n}=N_{n}\left(P_{n}\right)=\min _{P \in \mathscr{P}_{n}^{*}} N_{n}(P) .
$$

Определим сходимость в классе $\mathscr{F}$. Будем писать $f_{n} \stackrel{\mathscr{F}}{\longrightarrow} f$, если все $f_{n}$ и $f$ принадлежат $\mathscr{F}$ и выполнены следующие условия:

а) для любого отрезка $\Delta \subset F$ имеем: $f_{n} \rightarrow f$ по мере (на $\left.\Delta\right)$ и

$$
\lim _{n \rightarrow \infty} \min _{\Delta} f_{n}(x)=\min _{\Delta} f(x),
$$

b) выполнено соотношение

$$
\varliminf_{|x| \rightarrow \infty, n \rightarrow \infty} \frac{f_{n}(x)}{\log |x|}>1 .
$$

Подчеркнем, что условие b) (так же как и условие b) в определении класса $\mathscr{F}$, см. (66)) относится только к случаю неограниченных $F$. Знак “ $\rightarrow$ " в применении к последовательностям мер означает слабую сходимость: $\mu_{n} \rightarrow \mu$, если $\int \varphi d \mu_{n} \rightarrow \int \varphi d \mu$ для любой непрерывной финитной функции $\varphi$. Из известных свойств сходимости последовательностей потенциалов мер вытекает, что если $F$ - отрезок и $\mu_{n} \rightarrow \mu$, то $V^{\mu_{n}} \stackrel{\mathscr{F}}{\longrightarrow} V^{\mu}$; нетрудно показать также, что если $\mu_{n} \rightarrow \mu$ и $f_{n} \stackrel{\mathscr{F}}{\longrightarrow} f$, то $V^{\mu_{n}}+f \stackrel{\mathscr{F}}{\longrightarrow} V^{\mu}+f(F-$ отрезок, $\mu$ - мера с условием $(64))$.

Единичная мера, ассоциированная с многочленом $P, \operatorname{deg} P=n$, определяется по формуле

$$
\mu(P)=\frac{1}{n} \sum_{\zeta} \delta_{\zeta},
$$

где $\delta_{\zeta}$ - единичная мера с носителем в точке $\zeta$, сумма берется по всем нулям многочлена $P$ (с учетом их кратностей).

Меру, ассоциированную с экстремальным многочленом $P_{n}$, обозначим через $\mu_{n}: \mu_{n}=\mu\left(P_{n}\right), n=1,2, \ldots$ Ясно, что нули многочлена $P_{n}$ принадлежат $F$; следовательно, $\mu_{n} \in M(F), n=1,2, \ldots$.

TEOPEMA 6 (см. [47]). Если $f_{n} \stackrel{\mathscr{F}}{\longrightarrow} f$, mo

$$
\begin{gathered}
\mu_{n} \rightarrow \lambda \quad \text { nри } n \rightarrow \infty \\
\lim _{n \rightarrow \infty} N_{n}^{1 / n}=e^{-w}
\end{gathered}
$$

где $\lambda=\lambda(f), w=w(f)$. 
Равновесная мера не имеет атомов, поэтому соотношение (87) эквивалентно следующему утверждению: для любого отрезка $\Delta \subset F$

$$
\lim _{n \rightarrow \infty} \frac{1}{n} \varkappa_{n}(\Delta)=\int_{\Delta} d \lambda
$$

где $\varkappa_{n}(\Delta)$ - число нулей многочлена $P_{n}$ на отрезке $\Delta$.

В случае, когда $F$ - отрезок, из (87) вытекает асимптотическая формула

$$
\lim _{n \rightarrow \infty}\left|P_{n}(z)\right|^{1 / n}=e^{-V^{\lambda}(z)}, \quad z \in \mathbb{C} \backslash F
$$

с учетом (88) для нормированных экстремальных многочленов $P_{n}^{*}$ получаем

$$
\lim _{n \rightarrow \infty}\left|P_{n}^{*}(z)\right|^{1 / n}=e^{w-V^{\lambda}(z)}, \quad z \in \mathbb{C} \backslash F .
$$

Переход к пределу в последних двух формулах - равномерный внутри (на компактных подмножествах) области $\mathbb{C} \backslash F$.

Сказанное выше распространяется на случай, когда $F$ - объединение конечного числа промежутков прямой $\mathbb{R}$. В этом случае нельзя утверждать, что все нули экстремальных многочленов $P_{n}$ принадлежат $F$, но асимптотические соотношения (87) и (88) остаются в силе.

4.3. В приложениях, связанных с изучением асимптотических свойств различных характеристик случайных матриц, наиболее часто встречается ситуация, когда внешнее поле $f(x)$ - вещественная аналитическая функция на $\mathbb{R}$; например, $f(x)=Q(x)$ - полином четной степени с положительным старшим коэффициентом (см. прежде всего [58]). В этом случае носитель равновесной меры $S_{\lambda}$ состоит из конечного числа отрезков, $S_{\lambda}=\bigsqcup_{j=1}^{p}\left[e_{2 j-1}, e_{2 j}\right]=E$, a cама равновесная мера абсолютно непрерывна относительно меры Лебега на $E$ и имеет следующий вид:

$$
d \lambda(x)=\frac{\sqrt{H(x)}_{+}}{\pi} \rho(x) d x, \quad \rho(x)=\int_{E} \frac{Q^{\prime}(t)}{x-t} \frac{d t}{\sqrt{H(t)}+}, \quad H(z)=\prod_{j=1}^{2 p}\left(z-e_{j}\right),
$$

где под $\sqrt{H(x)}+$ на $E$ понимаются предельные значения из верхней полуплоскости той ветви корня, которая удовлетворяет условию $\sqrt{H(z)} \sim z^{p}$ при $z \rightarrow \infty$. При этом для концевых точек отрезков выполняются соотношения

$$
\begin{gathered}
\int_{E} \frac{Q^{\prime}(t) t^{k}}{\sqrt{H(t)}} d t=0, \quad k=0,1, \ldots, p-1, \\
\frac{i}{2 \pi} \int_{E} \frac{Q^{\prime}(t) t^{p}}{\sqrt{H(t)}} d t=1
\end{gathered}
$$

и

$$
\int_{e_{2 j}}^{e_{2 j+1}} \sqrt{H(x)}+\rho(x) d x=0, \quad j=1,2, \ldots, p-1 .
$$


Отметим, что задача нахождения в явном виде концевых точек неизвестных отрезков ${ }^{17}$ по заданному полиному $Q(x)$ в общем случае до сих пор не решена (см. выше пп. 4.2.1, 4.2.2).

4.4. Пусть теперь $t \in(0,+\infty)$ - произвольный вещественный параметр, $M_{t}=M_{t}(\mathbb{R})$ - семейство (положительных конечных борелевских) мер $\mu$ на $\mathbb{R}$ таких, что $\mu(\mathbb{R})=t ; \lambda_{t} \in M_{t}$ - равновесная мера, соответствующая внешнему полю $f$. Однопараметрическое семейство мер $\lambda_{t}(f), t \in \mathbb{R}_{+}$, называется семейством равновесных мер, соответствующих (фиксированному) внешнему полю $f$. В работе В.С. Буярова и Е. А. Рахманова [166] исследована зависимость семейств $\lambda_{t}, S_{\lambda_{t}}$ и $S^{\lambda_{t}}$, а также экстремальной константы $w\left(\lambda_{t}\right)$ от параметра $t \in \mathbb{R}_{+}$и получен следующий результат.

Теорема 7 (см. [166]). Существует не более чем счетное множество $N \subset \mathbb{R}_{+}$такое, что для всех $t \in \mathbb{R}_{+} \backslash N$ имеем

1) семейство равновесных мер $\lambda_{t}$ монотонно возрастает, непрерывно в слабой топологии на $M_{t}$ и дифференцируемо по $t$ слева и справа на $\mathbb{R}_{+} ;$для соответствующих производных имеют место формуль

$$
\partial_{t} \lambda_{t-0}=\gamma_{t} \quad u \quad \partial_{t} \lambda_{t+0}=\gamma^{t}
$$

где $\gamma_{t} u \gamma^{t}$ - робеновские (чебышевские) распределения для компактов $S_{\lambda_{t}} u S^{\lambda_{t}}$ соответственно;

2) для равновесной меры $\lambda_{t}$ и экстремальной константы $w\left(\lambda_{t}\right)$ справедливы представления

$$
\lambda_{t}=\int_{0}^{t} \gamma_{\tau} d \tau \quad u \quad w\left(\lambda_{t}\right)=-\int_{0}^{t} \log \operatorname{cap}\left(S_{\lambda_{\tau}}\right) d \tau
$$

3) для $x \in \bigcup_{t>0} S_{\lambda_{t}}$ потенциал внешнего поля $f$ допускает представление

$$
f(x)=\int_{0}^{\infty} g_{\tau}(x, \infty) d \tau,
$$

где $g_{\tau}(x, \infty)$ - функиия Грина области $\overline{\mathbb{C}} \backslash S_{\lambda_{\tau}}$ с полюсом в бесконечности.

Формула Буярова-Рахманова (93) позволяет восстановить потенциал $f(x)$ по семейству носителей $S_{\lambda_{t}}$ равновесных мер $\lambda_{t}$ (см., например, [131]).

\section{5. Одна обратная теорема о диагональных аппроксимациях Паде}

В этом разделе мы придерживаемся обозначений работы [28].

5.1. Пусть

$$
f(z)=1+c_{1} z+c_{2} z^{2}+\cdots
$$

- произвольный (вообще говоря, формальный) степенной ряд. Рассмотрим главную диагональ таблицы Паде ряда (94):

$$
f_{n}(z)=[n / n]_{f}(z), \quad n=0,1,2, \ldots
$$

\footnotetext{
${ }^{17}$ Подчеркнем, что число $p$ этих отрезков тоже неизвестно.
} 
(см. раздел 2 введения); всюду в этом разделе будем предполагать, что при любом $n$ имеет место соотношение

$$
\left(f-f_{n}\right)(z)=A_{n} z^{2 n+1}+\cdots, \quad n \in \mathbb{N}
$$

(с точки зрения определения аппроксимаций Паде, принятого в [89] и [8], предположение (96) означает, что диагональные аппроксимации $[n / n]_{f}$ существуют для всех $n \in \mathbb{N})$.

Если последовательность (95) равномерно сходится внутри круга $U_{r}:|z|<r$, то предельная функция $\tilde{f}$ этой последовательности голоморфна в $U_{r}$ и ее разложение в ряд Тейлора (с центром в точке $z=0$ ) совпадает с (94); тем самым, в этом случае ряд (94) также сходится в $U_{r}$, причем $\tilde{f}(z)=f(z), z \in U_{r}$. Обратное, вообще говоря, неверно; очевидным препятствием для равномерной сходимости последовательности (95) внутри $U_{r}$ служат полюсы рациональных функций $f_{n}, n=1,2, \ldots$, предельные точки которых могут принадлежать $U_{r}$ (соответствующие примеры хорошо известны). Принципиальный характер имеет вопрос о том, есть ли другие препятствия для равномерной сходимости диагональных аппроксимаций Паде $\left\{f_{n}\right\}$ внутри $U_{r}$. Иначе говоря, что можно утверждать относительно области равномерной сходимости последовательности (95), если известно, что ряд (94) сходится в $U_{r}$ и диагональные аппроксимации Паде $f_{n}$ не имеют полюсов в этом круге $\left(f\right.$ и все $f_{n}$ голоморфны в $\left.U_{r}\right)$. В такой форме этот вопрос был поставлен Бейкером в [89; гл. 12, c. 183]; лучший из приведенных в [89] результатов утверждает, что в этом случае последовательность (95) равномерно сходится внутри круга $|z| \leqslant c r$, $c=1 / \sqrt{3}=0.577 \ldots$.

Один из основных результатов, полученных А. А. Гончаром в [28], заключается в том, что если функиии $f_{n}$ голоморфны (не имеют полюсов) в круге $U_{r}$, то последовательность (95) равномерно сходится внутри $U_{r}$. Этот вывод остается в силе и в предположении, что функции последовательности (95) голоморфны внутри $U_{r}$, т. е. для любого компакта $K \subset U_{r}$ и всех $n>n(K)$ функции $f_{n}$ не имеют полюсов на $K$ (полюсы диагональных аппроксимаций Паде не имеют предельных точек в $\left.U_{r}\right)$. Подчеркнем, что при этом априорное предположение о сходимости ряда $(94)$ в $U_{r}$ оказывается излишним: голоморфность $f$ в $U_{r}$ также является следствием голоморфности диагональных аппроксимаций Паде внутри этого круга.

Этот принцип: голоморфность $\Rightarrow$ равномерная сходимость (в применении к диагональным аппроксимациям Паде) носит весьма общий характер. Поскольку диагональные аппроксимации Паде инвариантны относительно дробно-линейных отображений вида $\ell: z \mapsto z /(\alpha z+\beta)$ (а именно, $(f \circ \ell)_{n}=f_{n} \circ \ell$, $n \in \mathbb{N})$, из сказанного выше уже следует, что он имеет место для любого открытого круга $U \ni 0$ и, тем самым, для любой области $\Omega$, являющейся объединением таких кругов (например, $\Omega=\mathbb{C} \backslash[a,+\infty), a>0)$. Тем самым, классическая теорема Маркова (см. раздел 2 введения) оказывается непосредственным следствием того факта, что знаменатели диагональных аппроксимаций Паде марковской функции являются ортогональными многочленами, все нули которых (полюсы аппроксимаций) расположены на отрезке ортогональности. 
В [29] показано, что указанный принцип справедлив и для других типов областей $\Omega \ni 0$, часто встречающихся в теории сходимости непрерывных дробей и аппроксимаций Паде.

5.2. Общий результат А. А. Гончара, полученный в [28], удобно сформулировать после замены $z \mapsto 1 / z$.

Положим

$$
F_{n}(z)=f_{n}\left(\frac{1}{z}\right)=[n / n]_{f}\left(\frac{1}{z}\right), \quad n=0,1,2, \ldots ;
$$

рациональная функция $F_{n}-n$-я диагональная аппроксимация Паде ряда

$$
F(z)=1+\frac{c_{1}}{z}+\frac{c_{2}}{z}+\cdots
$$

$\left(F_{n}(z)=[n / n]_{F}(z)\right)$. Предположение (96) означает, что

$$
\left(F-F_{n}\right)(z)=\frac{A_{n}}{z^{2 n+1}}+\cdots, \quad n \in \mathbb{N} .
$$

Все дальнейшие рассмотрения ведутся в расширенной комплексной плоскости $\overline{\mathbb{C}}$. Пусть $\Omega$ - область в $\overline{\mathbb{C}}$ и $\left\{R_{n}\right\}-$ произвольная последовательность рациональных функций. Следуя [28], будем писать:

$\left\{R_{n}\right\} \in \mathscr{H}(\Omega)$, если для любого компакта $K \subset \Omega$ бункиии $R_{n}(z)$ голоморфнь (не имеют полюсов) на $K$ для всех $n>n(K)$;

$\left\{R_{n}\right\} \in \mathscr{C}(\Omega)$, если последовательность $\left\{R_{n}\right\}$ равномерно сходится внутри (на компактных подмножествах) области $\Omega$.

Если $\Omega \ni \infty$ и $\left\{F_{n}\right\} \in \mathscr{C}(\Omega)$, то ряд (98) сходится в некоторой окрестности $D_{R}:|z|>R$ точки $z=\infty$; предельная функция последовательности (97) и сумма ряда $(98)$ в $D_{R}$ совпадают (ср. п. 5.1). Поэтому в рассматриваемом случае предельную функцию последовательности (97) будем обозначать через $F(z)$, $z \in \Omega$ (ряд (98) задает элемент функции $F$ в окрестности точки $z=\infty$; последовательность (97) определяет голоморфное продолжение этого элемента в область $\Omega$ ). Тем самым, $\left\{F_{n}\right\} \in \mathscr{C}(\Omega) \Rightarrow F \in \mathscr{H}(\Omega)$ (последнее означает, что ряд (98) определяет функцию, голоморфную в области $\Omega$; очевидно также, что $\left.\left\{F_{n}\right\} \in \mathscr{C}(\Omega) \Rightarrow\left\{F_{n}\right\} \in \mathscr{H}(\Omega)\right)$.

Ниже conv $(\cdot)$ - выпуклая оболочка.

Обозначим через $\mathscr{G}=\mathscr{G}_{\infty}$ класс всех областей $\Omega$ вида

$$
\infty \in \Omega=D \backslash E,
$$

где $D$ - область и $E$ - относительно замкнутое подмножество $D$, удовлетворяющие условиям

$$
\partial D \subset \partial \widetilde{D}, \quad \operatorname{cap} E=0,
$$

$\widetilde{D}$ - дополнение к $\operatorname{conv}(\partial D)$.

Основной результат работы [28] состоит в следующем.

Tеорема 8. Если $\Omega \in \mathscr{G}$, mo

$$
\left\{F_{n}\right\} \in \mathscr{H}(\Omega) \quad \Rightarrow \quad\left\{F_{n}\right\} \in \mathscr{C}(\Omega) .
$$

Знак $\Rightarrow$ можно заменить на $\Leftrightarrow$. С учетом вышесказанного из теоремы 8 вытекает следующее утверждение. 
СлЕДСТВИЕ 5.1. Если $\Omega \in \mathscr{G}$, mo

$$
\left\{F_{n}\right\} \in \mathscr{H}(\Omega) \quad \Rightarrow \quad F \in \mathscr{H}(\Omega) .
$$

5.3. Приведем схему доказательства теоремы 8, следуя [28].

Для произвольной $\Omega$ область геометрической сходимости последовательности $\left\{F_{n}\right\}$ может быть охарактеризована с помощью функции

$$
\Phi(z)=\varlimsup_{n \rightarrow \infty}\left|\left(F_{n+1}-F_{n}\right)(z)\right|^{1 / n}, \quad z \in \Omega .
$$

Эта функция непрерывна в $\Omega, \Phi(\infty)=0$ (тем самым, $\Phi(z)<1$ в некоторой окрестности точки $z=\infty)$ и $q(K)=\|\Phi\|_{K}$ для любого компакта $K \subset \Omega$. Максимальная область (принадлежащая $\Omega$ и содержащая точку $z=\infty$ ), внутри которой геометрически сходится последовательность $\left\{F_{n}\right\}$, совпадает с неограниченной компонентой $G$ открытого множества $\{z \in \Omega: \Phi(z)<1\}$. С одной стороны, это позволяет локализовать препятствия для равномерной сходимости $\left\{F_{n}\right\}$ внутри всей области $\Omega$, с другой - перейти от полиномов и рациональных функций к логарифмическим потенциалам ассоциированных с ними мер (зарядов). Как это обычно делается в работах А. А. Гончара, применение понятий и результатов теории потенциала существенно упрощает анализ задачи.

Фиксируя точку $z_{0} \in \Omega$, в определении $\Phi\left(z_{0}\right)$ можно перейти к подпоследовательности $\Lambda=\Lambda\left(z_{0}\right) \subset \mathbb{N}$, для которой

$$
\Phi\left(z_{0}\right)=\lim _{n \rightarrow \infty, n \in \Lambda}\left|\left(F_{n+1}-F_{n}\right)\left(z_{0}\right)\right|^{1 / n}
$$

и, вместе с тем, нули и полюсы рациональных функций $F_{n}$ и $F_{n+1}, n \in \Lambda$, имеют правильное асимптотическое поведение (ассоциированные с ними меры слабо сходятся). При этом существенно, что потенциалы предельных мер для нулей и полюсов функций $F_{n}$ и $F_{n+1}, n \in \Lambda$, совпадают: для полюсов - в области $\Omega$, для нулей - в области $G$ (тем самым, в любой области $g, \infty \in g \subset \Omega$, где уже установлено неравенство $\Phi(z)<1)$. Формулы, выражающие $\Phi\left(z_{0}\right)$ через потенциалы, содержат свободный параметр; за счет выбора этого параметра удается показать, что для областей $\Omega \in \mathscr{G}$ имеет место неравенство $\Phi(z)<1$, $z \in \Omega$. В узловом случае, когда $D-$ круговая область, содержащая точку $z=\infty$, доказательство носит индуктивный характер; устанавливается, что нет (локальных) препятствий для расширения кругов $D$ (с сохранением неравенства $\Phi(z)<1, z \in D)$ в пределах области $\Omega$.

Из следствия 5.1 вытекает справедливость гипотезы Бейкера [89; гл. 12, c. 183].

\section{6. О некоторых гипотезах в теории аппроксимаций Паде}

6.1. Гипотеза Бейкера и Грейвс-Морриса. В этом разделе мы придерживаемся обозначений работы [90].

6.1.1. Пусть $f$ - функция, голоморфная в точке $z=0$ и заданная своим разложением в степенной ряд

$$
f(z)=\sum_{k=0}^{\infty} c_{k} z^{k}, \quad z \in D_{0},
$$


$D_{0}:|z|<R_{0}-$ круг сходимости ряда (103). Для любого $m \in \mathbb{N}$ через $D_{m}$ : $|z|<R_{m}$ обозначим максимальный открытый круг с центром в нуле, в который $f$ продолжается как мероморфная функция, имеющая $\leqslant m$ полюсов $\left(R_{m}-\right.$ радиус $m$-мероморфности функции $\left.f ; 0<R_{0} \leqslant R_{m} \leqslant \infty\right)$. Пусть $[n / m]_{f}-$ аппроксимация Паде типа $(n, m)$ ряда $(103), n \in \mathbb{N}$.

В [8; гл. 6, §6.1] (см. также [88], [89]) Бейкером и Грейвс-Моррисом была предложена следующая гипотеза.

ГиПотеЗА 6.1. Для любых $f$ u $m$ существует последовательность вида

$$
[n / m]_{f}, \quad n \in \Lambda \subset \mathbb{N}, \quad \Lambda=\Lambda(f, m)
$$

(подпоследовательность т-й строки таблицы Паде функиии $f$ ), равномерно сходящаяся $\kappa f$ на компактах, принадлежащих $D_{m}$ и не содержащих полюсов функи,и $f$.

Число полюсов $f$ в $D_{m}$ обозначим через $\mu(0 \leqslant \mu \leqslant m)$. Если $\mu=m$, то гипотеза 6.1 справедлива с $\Lambda=\mathbb{N}$ (теорема Монтессу, см. [18] и раздел 2 введения). Из результатов Адамара [17] следует справедливость гипотезы 6.1 при $\mu=m-1$. Этим охватываются случаи $m=1$ (см. [8; гл. $6, \S 6.1])$ и $m=2$, $\mu=2,1$; в п. 6.1 .2 приводится простой пример, опровергающий сформулированную выше гипотезу уже для случая $m=2, \mu=0$.

В некоторых работах рассматривался вопрос о справедливости гипотезы 6.1 для целых функций $f$; в частности, Эдрей [167] доказал, что гипотеза справедлива в том случае, когда порядок $\lambda$ функции $f$ и номер $m$ связаны соотношением: $\lambda m(m-1)<2$.

В [90] доказана следующая теорема.

Tеорема 9. Пусть $f$ такова, что $D_{m}=\mathbb{C}\left(\right.$ m.е. $\left.R_{m}=\infty\right)$. Тогда существует последовательность вида (104), равномерно сходящаяся $\kappa f$ на любом компакте в $\mathbb{C}$, не содержащем полюсов функиии $f$.

В частности, гипотеза 6.1 справедлива для любой целой функции $f$ при любом $m \in \mathbb{N}$.

Отметим, что в [90] доказано следующее более сильное утверждение.

ТЕОрема 10. Для любых $f$ u $m \in \mathbb{N}$ существует последовательность вида (104), равномерно сходяшаяся $\kappa f$ на компактах, принадлежащих кругу $|z|<c R_{m}$ и не содержащих полюсов функции $f(c>0$ - константа, зависящая только от $\mathrm{m}$ ).

6.1.2. Приведем контрпример к гипотезе Бейкера-Грейвс-Морриса (см. [90]). Рассмотрим функцию

$$
f(z)=\frac{1+\sqrt[3]{2} z}{1-z^{3}}=1+\sqrt[3]{2} z+z^{3}+\sqrt[3]{2} z^{4}+\cdots .
$$

В этом случае $R_{0}=R_{1}=R_{2}=1, H_{n, 2} \neq 0$ для всех $n=0,1, \ldots$ и полюсы $\zeta_{n, 2, j}, j=1,2$, аппроксимаций Паде $[n / 2]$ легко вычисляются:

$\zeta_{n, 2, j}=\frac{1 \pm i \sqrt{3}}{2 \sqrt[3]{2}}$ при $j=3 k, \quad \frac{1 \pm 3}{2 \sqrt[3]{2}}$ при $j=3 k+1, \quad \frac{1 \pm \sqrt{3}}{2 \sqrt[3]{2}}$ при $j=3 k+2$. 
При любом $n$ функция $[n / 2]$ имеет по меньшей мере один полюс, модуль которого не превосходит $1 / \sqrt[3]{2}$; тем самым, не существует последовательностей вида $[n / 2], n \in \Lambda$, равномерно сходящихся в круге $|z| \leqslant 1 / \sqrt[3]{2}$ (компактно принадлежащем $\left.D_{0}=D_{1}=D_{2}=\{z:|z|<1\}\right)$.

6.2. Гипотеза Бейкера-Гаммеля-Уиллса. В теории аппроксимаций Паде центральную роль играют вопросы сходимости, причем наибольший интерес вызывают вопросы равномерной сходимости диагонали. К результатам положительного характера относятся приведенные выше теоремы Маркова, Гончара, Рахманова и др. Заметим, что теорема Маркова предполагает кроме голоморфности функции определенное дополнительное условие на нее. В общем случае, когда дополнительные условия отсутствуют, ожидать равномерной сходимости всей последовательности аппроксимаций Паде не приходится. Сохраняя значение верхнего предела, $\overline{\lim }_{n \rightarrow \infty}\left|f_{n}\right|^{1 / n}$, достигаемого по некоторой подпоследовательности $\Gamma=\Gamma(f) \subset \mathbb{N}$, нетрудно построить примеры функций с каким угодно поведением полюсов аппроксимаций Паде при $n \notin \Gamma$. Очевидно, что наличие полюсов у приближающих функций в исследуемой области несовместимо с их равномерной сходимостью в этой области. В частности, Валлин [168] построил пример целой функции такой, что $\varlimsup_{n \rightarrow \infty}\left|[n, n]_{f}(z)\right|=\infty$ при всех $z \neq 0$. Однако этот пример Валлина, как и многие другие ранее известные примеры, не опровергает того утверждения, что из последовательности диагональных аппроксимаций Паде можно выбрать подпоследовательность, равномерно сходящуюся к $f$ на компактах, лежащих в круге ее голоморфности. Это позволило Г. Бейкеру, Д. Гаммелю и Д. Уиллсу [169] высказать в 1961 г. следующую гипотезу.

ГиПотезА 6.2 (Бейкер-Гаммель-Уиллс). Пусть функция $f$ голоморфна в некоторой окрестности точки $z=0$ и мероморфна в круге $D=\{|z|<1\}$. Тогда найдется бесконечная подпоследовательность $\Lambda=\Lambda(f)$ натуральных чисел такая, что $[n / n]_{f} \rightarrow f$ nри $n \rightarrow \infty, n \in \Lambda$, равномерно на компактах, лежащих в $D$ и не содержащих полюсов $f$.

Некоторые недостаточно четко сформулированные высказывания, близкие к гипотезе Бейкера-Гаммеля-Уиллса, можно найти уже в работах самого Паде, опубликованных им в начале 20-го столетия. Впоследствии гипотеза Бейкера-Гаммеля-Уиллса приобрела широкую известность под названием "Падегипотезы" ("Padé conjecture").

C 1961 по 2001 г. Паде-гипотезу не удавалось ни доказать, ни опровергнуть. Результаты положительного характера были получены за этот период времени только лишь при некоторых дополнительных условиях на функцию.

В 2001 г. Д. Любински анонсировал (полное доказательство опубликовано [107] в 2003 г.) отрицательный ответ на Паде-гипотезу в ее общей постановке. А именно, он показал, что мероморфная функция $H_{q}$, задаваемая непрерывной дробью Роджерса-Рамануджана

$$
H_{q}(z)=1+\frac{q z \mid}{\mid 1}+\frac{q^{2} z \mid}{\mid 1}+\frac{q^{3} z \mid}{\mid 1}+\cdots,
$$


опровергает Паде-гипотезу при $q=\exp \left(\frac{4 \pi i}{99+\sqrt{5}}\right)$. Контрпример Любински основан на свойствах сходимости непрерывной дроби Роджерса-Рамануджана, о которых более подробно будет сказано ниже (гл. 3, раздел 3). Отметим, что свойства сходимости непрерывной дроби Роджерса-Рамануджана таковы, что при всех $q \in \mathbb{C}$ необходимым условием того, чтобы функция $H_{q}$ была контрпримером к Паде-гипотезе, является наличие у функции $H_{q}$ как минимум двух полюсов в круге $|z|<1$, различных по модулю и аргументу (это условие выполнено при $q=\exp \left(\frac{4 \pi i}{99+\sqrt{5}}\right)$ ). Поэтому ни при каком $q \in \mathbb{C}$ функция $H_{q}$ не может служить контрпримером к голоморфному (в определенном смысле самому интересному) варианту Паде-гипотезы, в котором предполагается, что функция $f$ голоморфна в круге $|z|<1$.

В. И. Буслаев в 2001 г. анонсировал [105] и в 2002 г. опубликовал [106] простой пример, опровергающий как голоморфный вариант Паде-гипотезы, так и гипотезу Шталя (см. ниже п. 6.4.1). Найденный контрпример задается гиперэллиптической функцией

$$
f(z)=\frac{-27+6 z^{2}+3(9+\zeta) z^{3}+\sqrt{81\left(3-(3+\zeta) z^{3}\right)^{2}+4 z^{6}}}{2 z\left(9+9 z+(9+\zeta) z^{2}\right)},
$$

где $\zeta=\sqrt[3]{1}=(-1+\sqrt{3} i) / 2$ и выбрана та ветвь функции $f$, для которой $f(0)=0$. Обоснование этого контрпримера опирается на разложение функции $f$ в непрерывную периодическую (с периодом 3 , так как $\zeta^{3}=1$ ) дробь

$$
f(z)=\frac{z \mid}{\mid 3-3 \zeta^{2} z}+\frac{\zeta z^{2} \mid}{\mid 3-3 \zeta^{4} z}+\frac{\zeta^{2} z^{2} \mid}{\mid 3-3 \zeta^{6} z}+\frac{\zeta^{3} z^{2} \mid}{\mid 3-3 \zeta^{8} z}+\cdots,
$$

последовательность подходящих дробей которой совпадает с диагональю таблицы аппроксимаций Паде функции $f$, и на свойства сходимости предельно периодических (и, в частности, периодических) непрерывных дробей. Подробнее об этих свойствах будет сказано ниже (гл. 3, раздел 1). В указанном контрпримере имеются три точки $z_{1}, z_{2}=\zeta z_{1}, z_{3}=\zeta^{2} z_{1}$, в которых

$$
[3 n+j / 3 n+j]_{f}\left(z_{j}\right)=\tilde{f}\left(z_{j}\right) \neq f\left(z_{j}\right), \quad n=0,1, \ldots, \quad j=1,2,3,
$$

где $\tilde{f}$ - другая ветвь гиперэллиптической функции (108).

\section{3. Гипотезы Гончара.}

6.3.1. В этом пункте обсуждаются гипотезы об асимптотическом поведении строчных последовательностей, высказанные А. А. Гончаром в [27]. Всюду в п. 6.3 мы придерживаемся обозначений и терминологии [27].

Пусть

$$
f(z)=\sum_{k=0}^{\infty} c_{k} z^{k}
$$

- произвольный (вообще говоря, формальный) степенной ряд, $n, m \in \mathbb{N}$ - произвольные натуральные числа, $[n / m]_{f}$ - аппроксимация Паде типа $(n, m)$ ряда $(110), \mathscr{P}_{n, m}=\left\{\zeta_{n, 1}, \ldots, \zeta_{n, m_{n}}\right\}, m_{n} \leqslant m,-$ множество конечных полюсов 
рациональной функции $[n / m]_{f}$. Всюду в дальнейшем $m \in \mathbb{N}$ предполагается фиксированным.

Фиксируем точку $a \in \mathbb{C}$ и обозначим через $\lambda(a)$ число точек $\zeta_{n, j} \in \mathscr{P}_{n, m}$, стремящихся к $a$ при $n \rightarrow \infty$. Точнее, пусть $\zeta_{n, j}$ перенумерованы (при каждом $n$ ) в порядке неубывания их расстояний от $a$. Тогда $\lambda(a)=0$, если $\zeta_{n, 1} \nrightarrow a$, и $\lambda(a)=\lambda$, если $\zeta_{n, \lambda} \rightarrow a, \zeta_{n, \lambda+1} \nrightarrow a$. Положим $\mathscr{L}_{m}=\left\{a \in \mathbb{C}^{*}: \lambda(a) \geqslant 1\right\}$. Пусть $\mu(a)$ есть число точек $\zeta_{n, j} \in \mathscr{P}_{n, m}$, стремящихся к $a$ со скоростью геометрической прогрессии, $\mathscr{P}_{m}=\left\{a \in \mathbb{C}^{*}: \mu(a) \geqslant 1\right\}$. Ясно, что $\mathscr{L}_{m} \supset \mathscr{P}_{m}$ и $\lambda(a) \geqslant \mu(a)$.

В [27] была высказана следующая гипотеза.

ГИПотезА 6.3. Если $а \in \mathscr{L}_{m}$, то $R_{m-1} \geqslant|a|$ и а-особая точка функuи $f(z), z \in D_{m-1}$.

При $m=1$ равенство $R_{0}=|a|$ очевидно (конечный полюс функции $[n / 1]_{f}$ вычисляется по формуле $\zeta_{n, 1}=c_{n} / c_{n+1}$ в предположении, что $c_{n} \cdot c_{n+1} \neq 0$, в противном случае $[n / 1]_{f}-$ полином); утверждение о том, что $a$ - особая точка функции $f(z), z \in D_{0}$, составляет содержание классической теоремы Фабри "об отношении" (см., например, [93]). Если $a \in \mathscr{P}_{m}$, то справедливость гипотезы 6.3 вытекает из теоремы Гончара (см. [27], а также [94]); при этом $a-$ полюс функции $f$.

Гипотезу 6.3 удается доказать лишь при тех или иных предположениях о поведении полюсов последовательности $[n / m]_{f}$ в целом. А именно, когда все конечные полюсы $\zeta_{n, 1}, \ldots, \zeta_{n, m}$ имеют пределы при $n \rightarrow \infty$. Справедливость гипотезы 6.3 в этом случае доказана С. П. Суетиным [94] (см. также раздел 2 введения).

Вопрос о справедливости гипотезы 6.3 в общем случае остается открытым.

Гипотеза, аналогичная гипотезе 6.3, была высказана А. А. Гончаром (в устном виде) и для многоточечных аппроксимаций Паде. При дополнительном предположении о сходимости всех полюсов $m$-й строки таблицы многоточечных аппроксимаций Паде со скоростью геометрической прогрессии гипотеза была доказана А. А. Гончаром (без последующей публикации). В. И. Буслаев [170], [171] снял в этой теореме Гончара дополнительное предположение о геометрической скорости сходимости и доказал ее не только для многоточечных, но и для ортогональных аппроксимаций Паде и аппроксимаций Паде-Фабера (см. гл. 3, раздел 4).

В [27] сформулированы еще две гипотезы, непосредственно примыкающие к результатам раздела 2 введения.

ГиПотезА 6.4. Для любой точки $а \in \mathscr{L}_{m}$ справедлива следующая формула: $R_{m}=|a| / \Delta(a)$.

Отметим, что из результатов работы [27] вытекает, что это утверждение эквивалентно следующему: если $a \in \mathscr{L}_{m} \backslash \mathscr{P}_{m}$, mo $R_{m}=|a|$.

ГипотезА 6.5. Если $0 \neq a \in D_{m}, \operatorname{mo~} \lambda(a)=\mu(a)$.

Гипотезы 6.4 и 6.5 доказаны С. П. Суетиным [94].

В следующих двух пунктах излагаются гипотезы, выдвинутые А. А. Гончаром в [24]. Часть из них к настоящему времени доказана, остальные остаются 
открытыми до сих пор. Всюду в этих пунктах мы по возможности придерживаемся обозначений и терминологии [24].

6.3.2. Локальные аппроксимации. Пусть

$$
f(z)=\sum_{k=0}^{\infty} \frac{c_{k}}{z^{k}}, \quad|z|>R_{f}, \quad R_{f}=\varlimsup_{k \rightarrow \infty}\left|c_{k}\right|^{1 / k}<+\infty,
$$

$\mathfrak{f}$ - полная аналитическая функция, соответствующая элементу $f$ (точнее, элементу $\left.(f, D), D:|z|>R_{f}\right)$. Для любого $n \in \mathbb{N}$ положим $\nu_{n}(f)=\sup \{\nu(f-r)$ : $\left.r \in \mathscr{R}_{n}\right\}$, где $\nu(\cdot)$ - порядок нуля указанной функции в точке $z=\infty, \mathscr{R}_{n}-$ множество всех рациональных функций порядка не выше $n$.

При любом $n$ существует единственная функция $f_{n}, f_{n} \in \mathscr{R}_{n}$, такая, что $\nu_{n}(f)=\nu\left(f-f_{n}\right) ; f_{n}=[n / n]_{f}-n$-я диагональная аппроксимация Паде ряда (111). Если $a, a>1$, фиксировано (произвольным образом) и $|\cdot|=a^{-\nu(\cdot)}$, то $f_{n}$ - рациональная функция наилучшего приближения $f$ в классе $\mathscr{R}_{n}$ относительно этой нормировки: $\left|f-f_{n}\right|=\inf \left\{|f-r|: r \in \mathscr{R}_{n}\right\}$.

Для любого ряда (111) имеем (ниже $\Lambda$ - некоторое бесконечное подмножество $\mathbb{N}$, зависящее от $f)$ :

$$
\nu_{n}(f)>n, \quad n \in \mathbb{N} ; \quad \nu_{n}(f)>2 n, \quad n \in \Lambda, \quad \Lambda \subset \mathbb{N} .
$$

Функциональный аналог известной теоремы Туэ-Зигеля-Рота (см. [111], [112], [172; теорема 2]) для аппроксимаций Паде может быть сформулирован так: если $f$ - элемент алгебраической функиии, отличной от рациональной, то при любом $\varkappa, \varkappa>2$, неравенство $\nu_{n}(f)>\varkappa n$ имеет место лишь для конечного множества индексов $n$. Отсюда уже легко следует, что в таком случае

$$
\lim _{n \rightarrow \infty} \frac{\nu_{n}(f)}{n}=2 .
$$

В [24] были высказаны следующие гипотезы.

ГиПотезА 6.6. Если $f$ - элемент многозначной аналитической функции $\mathfrak{f}$, имеющей конечное множество особых точек, то справедливо (113).

Справедливость этой гипотезы вытекает из результатов Шталя [173], [70].

В [24] было высказано предположение, что для алгебраических функций утверждение гипотезы 6.6 может быть существенно усилено.

ГиПотеЗА 6.7. Если $f$ - элемент алгебраической функции $\mathfrak{f}$, отличной от рациональной, то $\left\{\nu_{n}(f)-2 n\right\}$ - ограниченная последовательность.

В общем случае эта гипотеза остается недоказанной до сих пор (ср. [174; §3, гипотеза 4]). Для алгебраических функций второго порядка ее справедливость вытекает из функциональной теоремы Лиувилля (см. [172; теорема 1]).

В связи с гипотезой 6.6 отметим, что если

$$
\varlimsup_{n \rightarrow \infty} \frac{\nu_{n}(f)}{n}=+\infty
$$


то $\mathfrak{f}$ - однозначная аналитическая функция; при этом, каково бы ни было конечное $A, A>0$, соотношение $\overline{\lim }_{n \rightarrow \infty} n^{-1} \nu_{n}(f)>A$ совместимо с многозначностью $\mathfrak{f}$ (первое из этих утверждений, по существу, содержится в [20], [23], второе вытекает из результатов Пойа [175]).

Сказанное выше можно переформулировать в терминах последовательностей нормальных индексов диагональных аппроксимаций Паде (см. [8], [21]); по существу, речь идет о возможных “лакунах" в последовательности ганкелевых определителей:

$$
H_{n}=\left|\begin{array}{cccc}
c_{1} & c_{2} & \ldots & c_{n} \\
c_{2} & c_{3} & \ldots & c_{n+1} \\
\ldots \ldots \ldots \ldots \ldots \ldots \ldots & \ldots \ldots \\
c_{n+1} & c_{n+2} & \ldots & c_{2 n}
\end{array}\right| .
$$

Так, соотношение (113) эквивалентно тому, что последовательность $\left\{H_{n}\right\}$ не имеет "лакун Адамара", а (114) означает, что $\left\{F_{n}\right\}$ имеет “лакуны Островского" (в терминологии книги [93]). По-видимому, многие результаты о лакунарных степенных рядах (см. [93]) имеют свои аналоги для диагональных аппроксимаций Паде.

6.3.3. Равномерные аппроксимации. Следуя [24], ограничимся формулировкой соответствующих задач для приближений функций $f$ вида (111) на кругах с центром в точке $z=\infty$. Пусть $R>R_{f}, E=\{z:|z| \geqslant R\}$ (f - голоморфная функция на $E)$ и $D_{R}=\{z:|z|<R\}$. Обозначим через $\rho_{n}(f)$ наилучшее приближение $f$ на $E$ в классе $\mathscr{R}_{n}$ :

$$
\rho_{n}(f)=\inf \left\{\|f-r\|_{E}: r \in \mathscr{R}_{n}\right\},
$$

$\|\cdot\|$ - sup-норма на $E$.

Пусть $\mathscr{K}_{f}$ - множество всех компактов $K, K \subset D_{R}$ (со связным дополнением) таких, что $f$ допускает голоморфное (однозначное аналитическое) продолжение в область $\mathbb{C} \backslash K$. Обозначим через $c_{D}(K)$ гринову емкость $K$ относительно $D_{R}$ (емкость конденсатора $\left.(K, E)\right)$; положим

$$
a_{f}=\sup \left\{\exp \frac{1}{c_{D}(K)}: K \in \mathscr{K}_{f}\right\} .
$$

Для любой $f$ имеем

$$
\varlimsup_{n \rightarrow \infty} \rho_{n}(f)^{1 / n} \leqslant \frac{1}{a_{f}} ;
$$

это неравенство вытекает из результатов Дж. Уолша [176; гл. VIII].

В [24] была высказана следующая гипотеза.

ГиПотезА 6.8. Для любой $f$

$$
\varliminf_{n \rightarrow \infty} \rho_{n}(f)^{1 / n} \leqslant \frac{1}{a_{f}^{2}} .
$$

В сформулированном здесь виде (для компакта $E=\{|z| \geqslant R\}$ ) гипотеза 6.8 была доказана О.Г. Парфеновым [177], в общем случае (для произвольного компакта $E$ ) - В. А. Прохоровым [178]-[180]. 
Неравенства (115), (116) являются аналогами неравенств (112). Чтобы яснее была аналогия с локальным случаем, нужно перейти в п. 6.3 .2 от $\nu_{n}$ к наилучшим приближениям $\lambda_{n}=\left|f-f_{n}\right|$. В частности, соотношение (113) тогда запишется так:

$$
\lim _{n \rightarrow \infty} \lambda_{n}(f)^{1 / n}=\frac{1}{a^{2}} .
$$

Аналогами гипотез 6.6, 6.7 являются следующие гипотезы.

ГиПотЕзА 6.9. Если $f$ - элемент аналитической функции $\mathfrak{f}$, имеющей конечное множество особых точек, то (ср. (117))

$$
\lim _{n \rightarrow \infty} \rho_{n}(f)^{1 / n}=\frac{1}{a_{f}^{2}} .
$$

Если, в условиях этой гипотезы, $\mathfrak{f}$ - однозначная аналитическая функция, то обе части равенства (118), очевидно, равны нулю.

Справедливость гипотезы 6.9 вытекает из результатов Шталя [173], [70].

ГиПотЕзА 6.10. Если $f$ - элемент алгебраической функиии $\mathfrak{f}$, отличной от рациональной, то

$$
c_{1}<\rho_{n}(f) \cdot a_{f}^{2 n}<c_{2},
$$

где $c_{1}, c_{2}$ - положительные постоянные (не зависящие от $n$ ).

Эта гипотеза остается недоказанной до сих пор (ср. с [174; §3, гипотеза 4] и с результатом А.И. Аптекарева - раздел 1 введения, формула (7)).

\section{4. Некоторые другие гипотезы.}

6.4.1. Гипотезы Шталя. В 1997 г. Г. Шталь [174] высказал несколько гипотез об асимптотическом поведении диагональных аппроксимаций Паде. В частности, он предложил два специальных варианта Паде-гипотезы (см. [174; гипотезы 6 и 7]). В одном из этих вариантов на функцию $f$ налагается дополнительное условие ее алгебраичности, а во втором - еще более сильное условие ее гиперэллиптичности. Напомним, что гиперэллиптической функиией называется алгебраическая функция вида $f(z)=r_{1}(z)+r_{2}(z) \sqrt{H(z)}$, где $H$ - многочлен, $r_{1}, r_{2}$ - рациональные функции. Число $g=[(m-1) / 2]$, где $m$ - степень многочлена $H$, называется родом гиперэллиптической функиии. Приведем здесь формулировку второй (более специализированной и казавшейся поэтому более достоверной) из гипотез Шталя.

ГиПотеЗА 6.11. Пусть функиия $f$ гиперэллиптична и мероморфна в единичном круге $D=\{|z|<1\}$. Тогда найдется бесконечная подпоследовательность $\Lambda=\Lambda(f) \subseteq \mathbb{N}$ натуральных чисел такал, что $[n / n]_{f} \rightarrow f$ рри $n \rightarrow \infty$, $n \in \Lambda$, равномерно на компактах, лежаших в $D$ u не содержащих полюсов $f$.

Заметим, что Г. Шталь, высказывая свои гипотезы, имел весьма серьезные аргументы в поддержку их справедливости. Один из веских аргументов в пользу гипотез - это доказанная Г. Шталем в [63]-[67] теорема о сходимости по емкости диагональных аппроксимаций Паде алгебраических функций. Еще более веский аргумент заключается в том, что вышеприведенная гипотеза доказана 
Г. Шталем в 1996 г. в [181] в ситуации "общего положения" и при некотором дополнительном условии на нули и полюсы рациональных функций $r_{1}$ и $r_{2}$.

В 2000 г. С. П. Суетин доказал в [98] Паде-гипотезу при том же дополнительном условии, что и у Г. Шталя, и также в ситуации “общего положения", но для функций более общего, по сравнению с гиперэллиптическими, вида, а именно для функций вида

$$
\widehat{\rho}(z)=\int_{S} \frac{\rho(\zeta) d \zeta}{(z-\zeta) \sqrt{H(\zeta)_{+}}},
$$

где $H$ - многочлен четной степени, а "симметричный" компакт $S$ комплексной плоскости и весовая функция $\rho(\zeta)$ удовлетворяют некоторым достаточно естественным требованиям (см. раздел 2).

Отметим, что в контрпримере Любински к Паде-гипотезе функция $H_{q}(107)$ не является алгебраической и, следовательно, не опровергает ни одну из гипотез Шталя. Функция $f$ в контрпримере Буслаева к Паде-гипотезе является гиперэллиптической (рода $g=2$ ) и опровергает пять из семи гипотез, предложенных Шталем в [174] (отметим, что 6-я гипотеза, как указано в [174], принадлежит Наттоллу). Приведем оставшиеся две открытые гипотезы из [174] (гипотезы 4 и 6 в нумерации [174]).

ГиПотЕзА 6.12. Если функиия $f$ мероморфна в единичном круге $D:|z|<1$, то найдется бесконечная подпоследовательность $\Lambda=\Lambda(f) \subseteq \mathbb{N}$ натуральных чисел такая, что $[n / n]_{f} \rightarrow f$ по емкости при $n \rightarrow \infty, n \in \Lambda$, на компактах, лежсащих в $D$.

ГИПОТЕЗА 6.13. Пусть $f$-алгебраическая функиия, голоморфная в точке $z=0$. Тогда найдется некоторое число $N=N(f) \in \mathbb{N}$ такое, что при каждом $n \in \mathbb{N}$ общее число (с учетом кратностей) ложных полюсов рациональной функиии $[n / n]_{f}$ не превосходит $N(f)$.

В [174] было отмечено, что во многих известных случаях $N(f)=g$ - род соответствующей $f$ римановой поверхности (ср. с гипотезой 6.7 Гончара).

6.4.2. Гипотезы Наттола. Гипотезы Наттолла оказали самое существенное влияние на развитие теории сходимости аппроксимаций Паде.

Наиболее известная из его гипотез (см. прежде всего [40]) - гипотеза о существовании и структуре допустимого компакта минимальной емкости для функции с конечным числом точек ветвления и о сходимости по емкости диагональных аппроксимаций Паде в дополнении к этому компакту. Первые результаты в этом направлении были получены самим Наттоллом (в том числе - с соавторами; см. [35], [36], [40]). Окончательно вопрос был решен Шталем [63]-[67] (см. также [70]).

В работах [38] и [40] Наттоллом было высказано несколько эвристических утверждений об асимптотическом поведении аппроксимаций Паде и ЭрмитаПаде. В настоящее время эти утверждения обычно рассматриваются как "гипотезы Наттолла".

6.4.3. Некоторые другие гипотезы. Из теоремы Шталя (см. раздел 3), в частности, вытекает, что каждый полюс функции $f$, мероморфной в области Шталя $D_{f}$, притягивает по меньшей мере столько полюсов $[n / n]_{f}$, какова его 
кратность: $\lambda(a) \geqslant \nu(a)$ для любой $a \in D$. В [98] показано, что для "типичных" гиперэллиптических функций в последнем соотношении имеет место знак равенства. Знак равенства имеет место и для функции Буслаева, опровергающей гипотезу Бейкера-Гаммеля-Уиллса. Естественно поэтому высказать следующую общую гипотезу.

ГиПотеЗА 6.14. Пусть $f$ - функиия, удовлетворяющая условиям теоремы Шталя, $D_{f}$ - соответствующая область Шталя. Тогда для любой точки $a \in D_{f}$ имеет место равенство: $\nu(a)=\lambda(a)$, где $\nu(a)$ - кратность полюса $f$ в точке $a, \lambda(a)$ - число полюсов $[n / n]_{f}$, притягивающихся $к а$.

В работе [96] было показано, что для функций марковского типа при некоторых дополнительных предположениях (см. раздел 1) поведение $m$ полюсов $[n / n]_{f}$ в малой окрестности $m$-кратного, $m \geqslant 3$, полюса $f$ уточняется следующим образом: асимптотически эти $m$ полюсов располагаются в вершинах правильного m-угольника. Этот результат справедлив и для простейших алгебраических функций вида $f(z)=r_{1}(z) / \sqrt{z^{2}-1}+r_{2}(z), z \in D$, при надлежащих ограничениях на рациональные функции $r_{1}, r_{2}$.

Естественно предположить, что для алгебраических функций утверждение гипотезы 6.14 может быть существенно усилено.

ГиПотезА 6.15. Пусть $f$-алгебраическая функиия, $D_{f}$ - соответствующая область Шталя. Тогда в малой окрестности любого $m$-кратного $(m \geqslant 3)$ полюса $а \in D_{f}$ функции $f$ существуют $m$ полюсов рациональной функции $[n / n]_{f}$, которые асимптотически располагаются в вершинах правильного т-угольника с иентром в точке $а$.

\section{Глава 2}

\section{Скорость рациональной аппроксимации аналитических функций}

\section{1. Основные понятия и постановка задачи}

1.1. Постановка задачи. Пусть

$$
f_{n}(z):=\int_{F} \frac{\Phi_{n}(t)}{t-z} \frac{d t}{2 \pi i}, \quad F \subset \mathbb{C}, \quad n \in \mathbb{N},
$$

- последовательность голоморфных в $\overline{\mathbb{C}} \backslash F$ функций, задаваемых интегралами типа Коши по жордановой спрямляемой дуге $F$, и

$$
\rho_{n}:=\rho_{n}\left(f_{n}, E\right):=\inf _{r \in \mathscr{R}_{n}}\left\{\max _{E}\left|f_{n}-r\right|\right\}, \quad E \cap F=\varnothing,
$$

- расстояние функции $f_{n}$ в равномерной норме на компакте $E$ до класса $\mathscr{R}_{n}$ рациональных функций $r$ порядка $\leqslant n$.

Настоящая глава посвящена скорости рациональной аппроксимации функций $f_{n}$, т. е. асимптотическому поведению $\rho_{n}$ при $n \rightarrow \infty$. Современная теория этой классической проблематики развита А. А. Гончаром (см. [19]-[31], [47]-[49], 
[71], [132]-[135], [137], [182], это далеко не полный перечень ключевых работ). В наиболее общих предположениях на $F, E$ и $\Phi_{n}$ результат, описывающий показатель скорости рациональной аппроксимации

$$
d:=\lim _{n \rightarrow \infty} \rho_{n}^{1 /(2 n)},
$$

получен в работе А. А. Гончара и Е. А. Рахманова [49].

Также в этой главе мы обсудим уточнение скорости приближения (122) для вещественно-симметричных функций (120), аппроксимируемых на промежутках (конечных или бесконечных интервалах) действительной оси. Речь идет о наличии и о величине предела

$$
\lim _{n \rightarrow \infty} \frac{\rho_{n}\left(f_{n}, E\right)}{\alpha_{n} d^{2 n}}, \quad \text { где } \alpha_{n}^{1 / n}=1+o(1),
$$

который называют точной константой приближения аналитической функции рациональными. Формулировки общей теоремы Гончара и Рахманова для функций вида (120) и полученных в [83] ее уточнений (теоремы 11 и 12) мы обсудим в следующем разделе 2 .

1.2. Асимптотика величины $\rho_{n}\left(e^{-x},[0, \infty)\right)$. Одним из замечательных приложений только что упомянутой общей теоремы из [49] явилось доказательство существования и нахождение величины предела

$$
d:=\rho\left(e^{-x},[0, \infty)\right)=\lim _{n \rightarrow \infty} \rho_{n}^{1 /(2 n)}\left(e^{-x},[0, \infty)\right) .
$$

Эта известная в свое время проблема (поставленная Р. Варгой в связи с численными методами решения уравнения теплопроводности [73], [72] и вызвавшая появление нескольких десятков работ, см. обзоры [74], [76]) решена в 1986 г. А. А. Гончаром и Е.А. Рахмановым (см. [71], [49]) было доказано, что предел (124) существует и равен $\sqrt{v}$, где $v$ - единственный положительный корень уравнения

$$
\sum_{k=1}^{\infty} a_{k} v^{k}=\frac{1}{8}, \quad a_{k}:=\left|\sum_{d \mid k}(-1)^{d} d\right| .
$$

Отметим, что в том же 1986 году, когда результаты [49] были анонсированы в докладе А. А. Гончара на Международном конгрессе математиков в Беркли (см. [71]), значение константы

$$
v=\frac{1}{9.2890254919208189 \ldots}
$$

независимо нашел А. Магнус (без строгого доказательства существования предела (124)). А. Магнус (см. [85], [84], [87]) описал постоянную $v$ в эквивалентной (125) форме:

$$
v=\exp \left(-\pi \frac{K^{\prime}}{K}\right), \quad K(k)=2 E(k)
$$

где $K$ и $K^{\prime}$ суть полные эллиптические интегралы 1-го рода для модулей $k$ и $k^{\prime}=\sqrt{1-k^{2}}$, а $E(k)$ есть полный эллиптический интеграл 2-го рода. 
Замечательный факт (также открытый Магнусом): оказывается, точное значение константы $v(126)$ найдено ранее в 1886 г. (ровно за сто лет!) в трактате Альфана [77] по эллиптическим функциям, где $v$ появилось как (единственный) положительный корень следующего уравнения, эквивалентного (125):

$$
\sum_{n=1}^{\infty} \frac{n v^{n}}{1-(-v)^{n}}=\frac{1}{8}
$$

Впоследствии А. Магнус (см. [86]), анализируя результаты численных экспериментов из [76], [75], выдвинул гипотезу о том, что

$$
\frac{\rho_{n}\left(e^{-x},[0, \infty)\right)}{d^{2 n}} \rightarrow 2 d \text { при } n \rightarrow \infty,
$$

т. е. константа, уточняющая показатель скорости рациональных аппроксимаций экспоненты, опять связана с величиной $v$ - постоянной Алъфана!

Справедливость гипотезы Магнуса следует из общей теоремы 12 об асимптотике (123), обсуждаемой в этой части. Редукция “теорема $12 \Rightarrow(127)$ " изложена в следующем пункте.

\section{3. Асимптотика многочленов, ортогональных с комплексным пе-} ременным весом. Важным инструментом исследования рациональных аппроксимаций является теория многочленов $P_{n}(z)=z^{n}+\cdots$, ортогональных на компакте $F$, состоящем из конечного числа (замкнутых) контуров и дуг в комплексной плоскости, относительно комплексного веса $h(z)$ :

$$
\int_{F} P_{n}(z) z^{\nu} h(z) d z=0, \quad \nu=0,1, \ldots, n-1 .
$$

Пожалуй, именно в цитированных выше работах по рациональным аппроксимациям А. А. Гончаром впервые были рассмотрены многочлены (128), ортогональные относительно переменного (зависящего от $n$ ) веса $h$ вида

$$
h(z)=h_{n}(z)=e^{-2 n Q(z)} .
$$

Их так называемая слабая асимптотика

$$
\left|P_{n}(z)\right|^{1 / n} \underset{n \rightarrow \infty}{\longrightarrow} \Phi(z)=\exp \left\{-V^{\lambda}(z)\right\}
$$

оказалась связана с логарифмическим потенциалом

$$
V^{\lambda}(z):=\int \log \frac{1}{|t-z|} d \lambda(t)
$$

равновесной меры $\lambda$, для которого выполняются соотношения равновесия во внешнем поле $\varphi:=\operatorname{Re} Q$ :

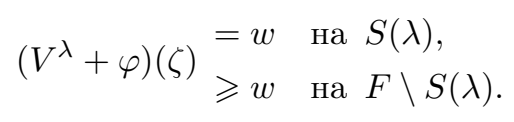

Необходимо отметить, что асимптотическая теория многочленов, ортогональных с переменным весом (129), переживает в настоящее время бурный расцвет, 
не только являясь эффективным инструментом для исследований в анализе и математической физике, но и находя применения в других разделах математики - от теории чисел до случайных матричных ансамблей и асимптотической комбинаторики (см., например, доклад П. Дейфта на Международном конгрессе математиков в Берлине [59]).

Возвращаясь к проблематике настоящей главы, заметим, что слабая асимптотика вида (130) для ортогональных многочленов решает задачу существования и определения величины предела (122) для показателей скорости наилучших рациональных аппроксимаций, в то время как для изучения более тонких пределов вида (123), являющихся нашей целью, требуются так называемые формулы сильной асимптотики вида

$$
\frac{P_{n}(z)}{\Phi^{n}(z)} \approx F_{n}(z),
$$

где функция $F_{n}$ может быть выражена, например, через стандартные функции некоторой римановой поверхности, соответствующей рассматриваемой задаче. Успех в определении точных констант (123) стал реальностью после недавнего существенного прогресса в развитии комплексных методов изучения сильных асимптотик. Краткий обзор этой тематики, а также формулировки доказанных в работе [83] теорем о сильной асимптотике многочленов, ортогональных относительно комплексного переменного веса [49; теоремы 2 и 3] мы приведем в следующем разделе.

\section{2. Формулировки теорем о скорости рациональной аппроксимации}

В этом разделе мы дадим необходимые определения и сформулируем теоремы о скорости наилучших рациональных аппроксимаций - теорему Гончара-Рахманова, а также полученные А.И. Аптекаревым в работе [83] некоторые ее уточнения. Затем мы обсудим приложения приведенных теорем, одно из которых состоит в доказательстве известной гипотезы Магнуса (см. (127)).

2.1. Равновесие симметричного конденсатора во внешнем поле. Теорема Гончара-Рахманова. Напомним основные понятия теории логарифмического потенциала во внешнем поле, привлеченные в [49] для описания исходных данных и ответа в задаче о показателе геометрической прогрессии в скорости аппроксимаций (122) (подробнее см. [49], [165], [51]).

Пусть $K$ - компакт в расширенной комплексной плоскости $\overline{\mathbb{C}}, M(K)$ - множество всех единичных положительных борелевских мер $\nu$ на $K$ (т. е. $S(\nu):=$ $\operatorname{supp} \nu \subseteq K)$ таких, что

$$
\int_{|t| \geqslant 1} \log |t| d \nu(t)<+\infty .
$$

Пара непересекающихся компактов положительной емкости $E$ и $F$ (пластины конденсатора),

$$
\operatorname{cap} E, \operatorname{cap} F>0, \quad E \cap F=\varnothing, \quad E \subset \overline{\mathbb{C}}, \quad F \Subset \mathbb{C},
$$

и функция $\varphi$, гармоническая в окрестности $\Omega$ пластины $F$,

$$
\varphi \in \operatorname{Harm} \Omega, \quad F \subset \Omega,
$$


будут обозначать конденсатор

$$
(E ; F, \varphi)
$$

в поле $\varphi$, действующем на пластину $F$.

Пусть $M(E, F)$ - множество всех зарядов $\mu=\mu_{F}-\mu_{E}, \mu_{E} \in M(E), \mu_{F} \in$ $M(F)$. Существует единственный заряд (см. [49])

$$
\lambda:=\lambda(E ; F, \varphi) \in M(E, F),
$$

минимизирующий функционал энергии

$$
J(\mu, \varphi):=\int\left(V^{\mu}+2 \varphi\right)(z) d \mu(z)
$$

в поле $\varphi$, где $\varphi \equiv 0$ на $E, \mu \in M(E, F), V^{\mu}$ обозначает логарифмический потенциал (131). Причем экстремальный заряд обладает следующим свойством равновесия:

$$
\begin{aligned}
\left(V^{\lambda}+\varphi\right)(z) & =w_{\Gamma} \quad \text { на } \Gamma:=S(\lambda) \cap F, \\
\geqslant w_{\Gamma} & \text { на } F, \\
V^{\lambda}(z) & =w_{E} \quad \text { на } E .
\end{aligned}
$$

Соотношения равновесия (138) однозначно определяют равновесный заряд $\lambda$ и константы равновесия $w_{\Gamma}, w_{E}$. Введем важное для дальнейшего обозначение

$$
w:=w(E ; F, \varphi)=w_{\Gamma}-w_{E} .
$$

Ключевую роль в задачах рациональных аппроксимаций играет следующее свойство "симметрии", введенное Г. Шталем в [63]-[67]. В случае, когда $Г=$ $S(\lambda) \subseteq F$ представляет собой объединение аналитических дуг, говорят, что конденсатор $(E ; F, \varphi)$ обладает $S$-свойством (или свойством симметрии) для пластины $F$ (т. е. $(E ; F, \varphi) \in S$ ), если выполняется следующее соотношение:

$$
\frac{\partial\left(V^{\lambda}+\varphi\right)}{\partial n_{+}}(\zeta)=\frac{\partial\left(V^{\lambda}+\varphi\right)}{\partial n_{-}}(\zeta), \quad \zeta \in \Gamma^{0},
$$

где $\Gamma^{0}$ - совокупность всех открытых дуг, составляющих Г. Как обычно, знаки “+" или “-" в нижнем индексе означают нормальные производные на Г, взятые с противоположных сторон.

Теперь мы можем привести формулировку основной теоремы Гончара и Рахманова (см. [49]).

Теорема 11. Дана последовательность аналитических функций (120),

$$
f_{n}(z):=\int_{F} \frac{\Phi_{n}(t)}{t-z} \frac{d t}{2 \pi i},
$$

рассмотрим отклонения $\rho_{n}$ ее наилучших рационалъных аппроксимачий (121) на множестве Е. Пусть

1) $E$ - объединение конечного числа континуумов ${ }^{18}$ в $\overline{\mathbb{C}}$;

2) $F$ - обгединение конечного числа спрямляемых дуг и кривых Жордана;

\footnotetext{
${ }^{18}$ Под континуумом здесь понимается связный компакт, содержащий более одной точки.
} 
3) $\Phi_{n} \in \mathscr{H}(\Omega), F \subset \Omega u \frac{1}{2 n} \log \frac{1}{\left|\Phi_{n}\right|} \underset{\Omega}{\rightrightarrows} \varphi$ при $n \rightarrow \infty, \varphi-$ гармоническая функиия;

Тогда

4) $(E ; F, \varphi) \in S$.

$$
\lim _{n \rightarrow \infty} \rho_{n}\left(f_{n}, E\right)^{1 / n}=d^{2}=e^{-2 w},
$$

где ш есть постоянная равновесия (139).

2.2. Краевая задача для комплексного потенциала. Чтобы сформулировать уточнение приведенной выше теоремы Гончара и Рахманова, касающееся существования предела (123), нам придется расширить понятие равновесия $S$-конденсатора.

Рассмотрим конденсатор

$$
\left(E, \varphi_{E} ; F, \varphi_{F}\right),
$$

на обе пластины которого действует гармоническое поле $\left(\varphi_{E}\right.$ на $E$ и $\varphi_{F}$ на $F$ соответственно). Пусть носители равновесного заряда $\lambda=\lambda_{F}-\lambda_{E}$ на обеих пластинах суть связные дуги $\Gamma_{E}$ и $\Gamma_{F}$ :

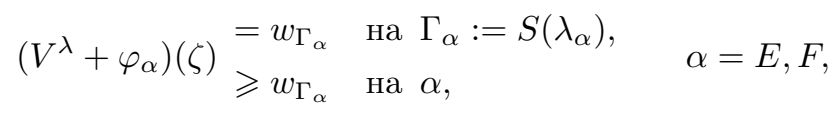

и пусть свойство симметрии $\left(E, \varphi_{E} ; F, \varphi_{F}\right) \in S$ в поле (140) выполнено на обеих пластинах:

$$
\frac{\partial\left(V^{\lambda}+\varphi_{\alpha}\right)}{\partial n_{+}}(\zeta)=\frac{\partial\left(V^{\lambda}+\varphi_{\alpha}\right)}{\partial n_{-}}(\zeta) \quad \text { на } \Gamma_{\alpha}^{0}, \quad \alpha=E, F
$$

Тогда для комплексного потенциала заряда $\lambda$

$$
\mathscr{V}^{\lambda}(z):=V^{\lambda}(z)+i \widetilde{V}^{\lambda}(z)=\int \log \frac{1}{t-z} d \lambda,
$$

определяемого с точностью до мнимой константы с помощью соотношений Коши-Римана, $S$-свойство (144) эквивалентно переписывается так:

$$
\begin{gathered}
\frac{d}{d z}\left(\mathscr{V}_{+}^{\lambda}+Q_{\alpha}\right)=-\frac{d}{d z}\left(\mathscr{V}_{-}^{\lambda}+Q_{\alpha}\right) \quad \text { на } \Gamma_{\alpha}, \quad \alpha=E, F, \\
Q_{\alpha}=\varphi_{\alpha}+i \widetilde{\varphi}_{\alpha}, \quad Q_{\alpha} \in \mathscr{H}\left(\Omega_{\alpha}\right), \quad \alpha=E, F .
\end{gathered}
$$

Это, в свою очередь, влечет следующую краевую задачу для комплексного потенциала:

$$
\mathscr{V}_{+}^{\lambda}+\mathscr{V}_{-}^{\lambda}= \begin{cases}-2 Q_{F}+2 W_{F} & \text { на } \Gamma_{F}, \\ -2 Q_{E}+2 W_{E} & \text { на } \Gamma_{E},\end{cases}
$$

где однозначно определенные действительные части констант $W_{\alpha}$

$$
w_{\Gamma_{\alpha}}^{*}:=\operatorname{Re} W_{\alpha}, \quad \alpha=E, F,
$$

ввиду соотношений равновесия (143) равны константам равновесия:

$$
w_{\Gamma_{\alpha}}^{*}=w_{\Gamma_{\alpha}}, \quad \alpha=E, F .
$$


Отметим, что эквивалентная переформулировка $S$-свойства (145) означает, что не только потенциал экстремального заряда находится в равновесии с полем на носителе, но и сопряженный потенциал находится в равновесии с сопряженным полем.

Таким образом, голоморфная функция

$$
f=\exp \left\{-\mathscr{V}^{\lambda}\right\}
$$

является решением следующей краевой задачи:

$$
\begin{gathered}
f \in \mathscr{H}^{\infty}\left(\overline{\mathbb{C}} \backslash\left(\Gamma_{F} \cup \Gamma_{E}\right)\right), \quad f \neq 0 \text { в } \overline{\mathbb{C}} \backslash\left(\Gamma_{F} \cup \Gamma_{E}\right), \\
f(\infty)=1, \quad \Delta_{F} \arg f=2 \pi, \quad \Delta_{E} \arg f=-2 \pi, \\
f_{+} f_{-}=\exp \left\{2 Q_{\alpha}-2 W_{\alpha}\right\} \quad \text { на } \Gamma_{\alpha}, \quad \alpha=E, F .
\end{gathered}
$$

Заметим, для любых исходных данных, а именно для

а) двух гладких непересекающихся дуг $\Gamma_{F}$ и $\Gamma_{E}$,

б) комплексных функций $Q_{F}$ и $Q_{E}$ из класса Гёльдера,

краевая задача (149) имеет единственное решение:

$$
\text { функцию } f \text { и константы } W_{F}, W_{E} \text {. }
$$

(Это утверждение сводится к стандартным фактам теории краевых задач, подробнее см. [83].)

Однако не для любых исходных данных решение задачи (149) представимо в виде (148), где $\lambda$ - равновесный заряд конденсатора (142). Как следует из единственности решения задачи (149) и из приведенных выше рассуждений, это имеет место только для исходных данных

$$
\left(\Gamma_{E}, \operatorname{Re} Q_{E} ; \Gamma_{F}, \operatorname{Re} Q_{F}\right) \in S,
$$

и тогда, сохраняя для (151) обозначения (146), мы будем иметь (147). В остальных случаях $\mathscr{V}^{\lambda}$ в (148) можно трактовать как комплексный потенциал комплексного заряда, равновесного в смысле соотношения (145).

По аналогии с (139) обозначим

$$
w^{*}:=w^{*}\left(\Gamma_{E}, Q_{E} ; \Gamma_{F}, Q_{F}\right):=w_{\Gamma_{F}}^{*}-w_{\Gamma_{E}}^{*} .
$$

Имеющиеся явные формулы для решения (151) задачи (149) дают выражение и для величины $w^{*}(152)$ (см. [83; §3, (3.4), (3.5)]).

2.3. Общая теорема о константе аппроксимаций. Гипотеза Магнуса. Справедлива следующая теорема.

ТЕОРема 12. Для заданной последовательности аналитических функщий

$$
f_{n}(z):=\int_{F} \frac{\Phi_{n}(t)}{t-z} \frac{d t}{2 \pi i}
$$

рассмотрим отклонения ее наилучших рациональных аппроксимаций (121) на множестве $E$

$$
\rho_{n}:=\rho_{n}\left(f_{n}, E\right)
$$


$\Pi y c m s$

1) $Е$ есть промежуток ${ }^{19} \mathbb{R}$;

2) $F$ есть $\mathbb{R}$-симметричная спрямляемая дуга Жордана $(F$ связно, $\bar{F}=F)$;

3) $\Phi_{n}$ есть вещественно-симметричная аналитическая в окрестности $\Omega$ дуги $F$ функиия такая, что

$$
\Phi_{n}(z):=\exp \left\{-2\left(n Q(z)+Q_{1}(z)\right)\right\}, \quad Q, Q_{1} \in \mathscr{H}(\Omega) ;
$$

4) $(E ; F, \operatorname{Re} Q) \in S$, множество равновесия на $F$

$$
\Gamma:=\left\{z: V^{\lambda(E ; F, \operatorname{Re} Q)}(z)+\operatorname{Re} Q(z)=w_{\Gamma}\right\}
$$

связно и $\lambda^{\prime}>0$ во внутренних точках дуги Г.

Тогда

$$
\rho_{n}=2 d^{*} d^{2 n}(1+o(1))
$$

əдe

$$
\begin{aligned}
d & =\exp \{-w(E ; F, \operatorname{Re} Q)\}=\exp \left\{-w^{*}(E ; \Gamma, Q)\right\}, \\
d^{*} & =\exp \left\{-w^{*}\left(E ; \Gamma, 2 Q_{1}\right)\right\},
\end{aligned}
$$

a $w^{*}$ (152) определяется параметрами решения краевой задачи (149).

ЗАмечание 2.1. Требование связности множества равновесия в условии 4) теоремы 12 является несколько более ограничительным, чем просто связность носителя $S(\lambda)$ равновесной меры $\lambda_{F}$. Достаточным условием выполнения 4) в (153) является выпуклость $\operatorname{Re} Q$ на $F$ (см., например, [47]).

Конечно, в теореме 12 сделаны гораздо более ограничительные предположения, чем в теореме Гончара-Рахманова. Часть из этих дополнительных ограничений носит технический характер (это касается $\mathbb{R}$-симметрии задачи или строгой положительности $\lambda^{\prime}$ внутри Г) и могла бы быть снята. Однако основное ограничение - связность носителя равновесной меры - представляется адекватным, если мы хотим иметь существование предела (123).

Из теоремы 12 вытекает следующий результат.

СлЕДСТвиЕ 2.1. В условиях теоремы 12 справедлива следующая импликаиця:

$$
\begin{gathered}
\Phi_{n}:=\exp \left\{-2\left(n+\frac{1}{2}\right) Q\right\} \\
\Downarrow \\
\rho_{n}=2 d d^{2 n}(1+o(1)), \quad d=\exp \{-w(E ; F, \operatorname{Re} Q)\} .
\end{gathered}
$$

Отсюда уже нетрудно извлечь справедливость гипотезы Магнуса. Действительно, следуя [49], имеем

$$
\rho_{n}\left(e^{-x},[0, \infty)\right)=\rho_{n}\left(e^{-(n+1 / 2) x},[0, \infty)\right) .
$$

Записываем $e^{-2(n+1 / 2) x}$ в виде интеграла Коши по контуру $\widetilde{F}$ в $\overline{\mathbb{C}}$, охватывающему $[0, \infty)$, и замечаем, что интегралы по концам $\widetilde{F}$ в окрестности точки $\infty$

\footnotetext{
${ }^{19}$ Промежуток есть конечный или полубесконечный интервал $\mathbb{R}$.
} 
не вносят существенного вклада в (157), поэтому

$$
\rho_{n}\left(e^{-x},[0, \infty)\right)=\rho_{n}\left(\int_{F} \frac{e^{-2(n+1 / 2) t / 2}}{t-x} d t,[0, \infty)\right)(1+o(1)),
$$

где $F$ - конечная дуга Жордана, которая, как показано в [49], может быть деформирована так, что условие 4) теоремы 12 выполнено. Таким образом, по следствию 2.1 из теоремы 12 гипотеза Магнуса (127) верна.

Аналогичное применение теоремы 12 к рациональным приближениям функции $x^{k} e^{-x}, k \in \mathbb{N}$, на $[0, \infty)$ дает в $(123)$ последовательность $\alpha_{n}:=n^{k}$, уточняющую скорость приближения, при этом

$$
\frac{\rho_{n}\left(x^{k} e^{-x},[0, \infty)\right)}{n^{k} d^{2 n}} \underset{n \rightarrow \infty}{\longrightarrow} 2 e^{-w^{*}}, \quad w^{*}:=w^{*}\left([0, \infty) ; F, \frac{k}{2} \log z\right),
$$

где $d^{2}=v$ - постоянная Альфана $(126)$ и для любого $k=0,1,2, \ldots$ компакт $F$ дуга Гончара-Рахманова (см. [49]), т. е. такая дуга, что $([0, \infty) ; F, \operatorname{Re} z / 2) \in S$.

\section{Глава 3}

\section{Непрерывные дроби и рекуррентные соотношения}

\section{1. Рекуррентные соотношения, теоремы Пуанкаре и Перрона и их приложения к теории сходимости непрерывных дробей}

K рекуррентным соотношениям

$$
f_{n}+\alpha_{1, n} f_{n-1}+\cdots+\alpha_{k, n} f_{n-k}=0, \quad n=k, k+1, \ldots,
$$

связывающим между собой элементы последовательности $\left\{f_{n}\right\}_{n=0}^{\infty}$, приводят многие задачи анализа и теории чисел. В частности, индукцией по числу $n$ легко проверяется, что последовательности числителей $\left\{P_{n}\right\}_{n=1}^{\infty}$ и знаменателей $\left\{Q_{n}\right\}_{n=1}^{\infty}$ числовой непрерывной дроби

$$
a_{0}+\frac{a_{1}}{b_{1}+\frac{a_{2}}{b_{2}+\ddots}}
$$

связаны между собой соотношениями

$$
P_{n}=b_{n} P_{n-1}+a_{n} P_{n-2}, \quad Q_{n}=b_{n} Q_{n-1}+a_{n} Q_{n-2}, \quad n=1,2, \ldots
$$

Другими словами, последовательности $\left\{P_{n}\right\}_{n=1}^{\infty}$ и $\left\{Q_{n}\right\}_{n=1}^{\infty}$ являются решениями одного и того же разностного уравнения

$$
X_{n}=b_{n} X_{n-1}+a_{n} X_{n-2} \quad n=1,2, \ldots,
$$

со следующими начальными условиями: $P_{-1}=1, P_{0}=a_{0}$ и $Q_{-1}=0, Q_{0}=1$. 
Легко видеть, что всякое решение разностного уравнения (158) однозначно определяется своими начальными условиями $f_{0}, \ldots, f_{k-1}$ и может быть найдено шаг за шагом из соотношений (158). Нетрудно проверить, что общее решение разностного уравнения (158) с постоянными коэффициентами $\alpha_{j, n}=\alpha_{j}$ $\left(n \geqslant n_{0}, j=1, \ldots, k\right)$ имеет следующий вид:

$$
f_{n}=\sum_{j=1}^{m} \lambda_{j}^{n}\left(C_{j, 1}+\cdots+C_{j, l_{j}} n^{l_{j}-1}\right), \quad n \geqslant n_{0}-k,
$$

где $\lambda_{1}, \ldots, \lambda_{m}$ - корни характеристического многочлена $h(z)=z^{k}+\alpha_{1} z^{k-1}+$ $\cdots+\alpha_{k}$ кратностей $l_{1}, \ldots, l_{m}$ соответственно, $l_{1}+\cdots+l_{m}=k$. Из явного вида (161) решений разностного уравнения (158) с постоянными коэффициентами следует, что если корни характеристического многочлена $h(z)$ различны по модулю, то существует предел $\lim _{n \rightarrow \infty} f_{n+1} / f_{n}$ и этот предел равен одному из корней характеристического многочлена. Оказывается, что это утверждение имеет место не только для разностных уравнений с постоянными коэффициентами, но и для разностных уравнений с предельно постоянными коэффициентами, когда найти решения в явном виде не представляется возможным. Соответствующее утверждение составляет содержание теоремы Пуанкаре [183] - одной из самых тонких в теории разностных уравнений.

ТЕОРемА ПУАнКАРЕ. Пусть последователъность $\left\{f_{n}\right\}_{n=0}^{\infty}$ является решением разностного уравнения (158) с предельно постоянными коэффициентами, корни характеристического многочлена

$$
h(z)=\lim _{n \rightarrow \infty}\left(z^{k}+\alpha_{1, n} z^{k-1}+\cdots+\alpha_{k, n}\right)
$$

которого различны по модулю. Тогда либо $f_{n}=0$ при всех $n \geqslant n_{0}$, либо существует предел $\lim _{n \rightarrow \infty} f_{n+1} / f_{n}$ u этот предел равен одному из корней характеристического многочлена.

Простые примеры показывают, что условия теоремы Пуанкаре нельзя ослабить, не нарушив утверждающей части теоремы, а утверждающую часть нельзя усилить, не потребовав дополнительных предположений. Ниже будут рассмотрены некоторые задачи, при решении которых весьма полезными оказываются те или иные уточнения теоремы Пуанкаре.

Весьма важное уточнение теоремы Пуанкаре было сделано О. Перроном [184]-[185] для невырожденных разностных уравнений. Напомним, что разностное уравнение (158) называется невырожденным, если $\alpha_{k, n} \neq 0$ при всех $n=k, k+1, \ldots$. Невырожденность уравнения (158) означает возможность его решения в "другую сторону", т. е. возможность однозначного определения значения $f_{n}$ при известных значениях $f_{n+1}, \ldots, f_{n+k}$.

ТЕОРема ПерРона. Пусть корни характеристического многочлена невырожденного разностного уравнения (158) с предельно постоянными коэфбициентами различны по модулю. Тогда для всякого корня $\lambda$ характеристического многочлена найдется решение $\left\{f_{n}\right\}_{n=0}^{\infty}$ разностного уравнения (158) такое, что $\lim _{n \rightarrow \infty} f_{n+1} / f_{n}=\lambda$. 
Первоначальное доказательство теоремы, данное О. Перроном в [184]-[185], было очень сложным и занимало свыше ста страниц текста. Позже М. А. Евграфов [186] указал значительно более короткое доказательство.

Теоремы Пуанкаре и Перрона могут быть записаны в векторном виде.

ТЕОРемА ПУАНКАРЕ-ПЕРрона в ВеКТорНом ВИДЕ. Пусть заданная последовательность $\left\{\bar{\omega}_{n}\right\}_{n=0}^{\infty}=\left\{\left(\omega_{n, 1}, \ldots, \omega_{n, k}\right)\right\}_{n=0}^{\infty} \quad k$-мерных векторов является решением векторного разностного уравнения

$$
\bar{\omega}_{n}=A_{n} \bar{\omega}_{n-1}, \quad n=1,2, \ldots,
$$

где $(k \times k)$-матрици $A_{n}$ имеют предел $\lim _{n \rightarrow \infty} A_{n}=A$, и пусть собственные значения пределъной матрицы $A$ различны по модулю. Тогда либо $\bar{\omega}_{n}=\overline{0}$ при всех $n \geqslant n_{0}$, либо при некотором $j \in\{1, \ldots, k\}$ существуют предель

$$
\lim _{n \rightarrow \infty} \frac{\omega_{n+1, j}}{\omega_{n, j}}=\lambda \quad u \quad \lim _{n \rightarrow \infty} \frac{\bar{\omega}_{n}}{\omega_{n, j}}=\bar{e},
$$

где $\lambda$ - собственное значение матрицы $A, \bar{e}$ - собственный вектор, соответствующий собственному значению $\lambda$.

Если разностное уравнение (163) невырождено (m.е. $\operatorname{det} A_{n} \neq 0$ при всех $n=1,2, \ldots), \lambda$ - наперед заданное собственное значение матрицы $A$, то найдется решение $\left\{\bar{\omega}_{n}\right\}_{n=0}^{\infty}$ векторного разностного уравнения (163), для которого выполняются равенства (164) при некотором $j \in\{1, \ldots, k\}$.

Если положить

$$
\left\{\left(f_{n-k+1}, \ldots, f_{n}\right)\right\}_{n=0}^{\infty}=\left\{\bar{\omega}_{n}\right\}_{n=0}^{\infty}=\left\{\left(\omega_{n, 1}, \ldots, \omega_{n, k}\right)\right\}_{n=0}^{\infty},
$$

то разностное уравнение (158) можно записать в виде векторного разностного уравнения (163) с матрицами $A_{n}$ специального вида

$$
A_{n}=\left(\begin{array}{ccccc}
0 & 1 & 0 & \ldots & 0 \\
0 & 0 & 1 & \ldots & 0 \\
\ldots \ldots & \ldots \ldots \ldots & \ldots \ldots \ldots \ldots & \ldots \\
0 & 0 & 0 & \ldots & 1 \\
-\alpha_{n, k} & -\alpha_{n, k-1} & -\alpha_{n, k-2} & \ldots & -\alpha_{n, 1}
\end{array}\right) .
$$

Формально это означает, что векторная теорема Пуанкаре-Перрона является более общей по сравнению с классическими теоремами Пуанкаре и Перрона, так как не имеет никаких ограничений на вид матриц $A_{n}$. Вместе с тем отметим, что доказательство теоремы Пуанкаре, приведенное в [187], специфичность вида (165) матриц $A_{n}$ практически не использует и может считаться доказательством и векторной теоремы Пуанкаре. Доказательство векторной теоремы Перрона дано в работе Г. Фреймана [188].

В [189] показано, что векторная формулировка теоремы Пуанкаре-Перрона допускает определенное уточнение при любом $k \geqslant 2$. Однако это уточнение наиболее естественно и просто формулируется в случае $k=2$, которым мы здесь и ограничимся. Именно этот случай представляет наибольший интерес с точки зрения исследования разностного уравнения (160) второго порядка, соответствующего непрерывной дроби (159). 
ТеОРема 13. Пусть имеется невырожденная линейная система разностных уравнений 2-го порядка

$$
\left\{\begin{array}{l}
\omega_{n}^{1}=\alpha_{n} \omega_{n-1}^{1}+\beta_{n} \omega_{n-1}^{2}, \\
\omega_{n}^{2}=\gamma_{n} \omega_{n-1}^{1}+\delta_{n} \omega_{n-1}^{2},
\end{array} \quad n=1,2, \ldots,\right.
$$

u пусть при всех $n=1,2, \ldots$

$$
\left|\alpha_{n}\right|+\left|\beta_{n}\right| \leqslant q\left(\left|\delta_{n}\right|-\left|\gamma_{n}\right|\right)
$$

где $q<1$. Тогда справедливы следующие утверждения.

$1^{\circ}$. Существует единственное (с точностъю до постоянного множителя) нетривиальное решение $\left\{\bar{\nu}_{n}\right\}_{n=0}^{\infty}=\left\{\left(\nu_{n}^{1}, \nu_{n}^{2}\right)\right\}_{n=0}^{\infty}$ системы (166) такое, что $\left|\nu_{n}^{1}\right| \geqslant\left|\nu_{n}^{2}\right|$ при всех $n=0,1, \ldots$.

При этом если $\lim _{n \rightarrow \infty} \gamma_{n} / \delta_{n}=0$, mо $\lim _{n \rightarrow \infty} \nu_{n}^{2} / \nu_{n}^{1}=0$.

$2^{\circ}$. Для всякого решения $\left\{\bar{u}_{n}\right\}_{n=0}^{\infty}=\left\{\left(u_{n}^{1}, u_{n}^{2}\right)\right\}_{n=0}^{\infty}$, отличного от исключительного решения $\left\{\bar{\nu}_{n}\right\}_{n=0}^{\infty}$, найдется индекс $n_{0}$ такой, что при всех $n \geqslant n_{0}$ выполняются неравенства

$$
\left|u_{n}^{2}\right|>\left|u_{n}^{1}\right|, \quad\left|\frac{\nu_{n}^{1}}{u_{n}^{2}}\right| \leqslant q^{n-n_{0}}\left|\frac{\nu_{n_{0}}^{1}}{u_{n_{0}}^{2}}\right|
$$

u, в частности, существует предел $\lim _{n \rightarrow \infty} \nu_{n}^{1} / u_{n}^{2}=0$.

При этом если $\lim _{n \rightarrow \infty} \beta_{n} /\left(\left|\delta_{n}\right|-\left|\gamma_{n}\right|\right)=0$, то $\lim _{n \rightarrow \infty} u_{n}^{1} / u_{n}^{2}=0$.

Приведенную теорему 13 можно рассматривать как уточнение двумерного векторного варианта теорем Пуанкаре и Перрона, так как общий случай двумерной теоремы Пуанкаре-Перрона линейной заменой сводится к случаю, когда существуют пределы

$$
\lim _{n \rightarrow \infty} \alpha_{n}=\alpha, \quad \lim _{n \rightarrow \infty} \beta_{n}=\lim _{n \rightarrow \infty} \gamma_{n}=0, \quad \lim _{n \rightarrow \infty} \delta_{n}=\delta, \quad|\alpha|<|\delta| .
$$

Применительно к рекуррентным соотношениям (160) второго порядка, играющим важную роль в теории непрерывных дробей, как следствия теоремы отметим следующие утверждения.

СЛЕДСТвИЕ 1.1. Пусть $a_{n} \neq 0$ при всех $n=1,2, \ldots$ и корни $\lambda_{n, 1} u \lambda_{n, 2}$ многочленов $z^{2}-b_{n} z-a_{n}$ такови, что $\lim _{n \rightarrow \infty} \lambda_{n, 1}=\lambda u \overline{\lim }_{n \rightarrow \infty}\left|\lambda_{n, 2}\right|<|\lambda|$. Тогда для всякого решения $\left\{f_{n}\right\}_{n=-1}^{\infty}$ разностного уравнения (160) за исключением единственного (с точностъю до постоянного множителя) решения $\left\{h_{n}\right\}_{n=-1}^{\infty}$ существует предел $\lim _{n \rightarrow \infty} f_{n+1} / f_{n}=\lambda$. При этом $\underline{\lim }_{n \rightarrow \infty}\left|h_{n+1} / h_{n}-\lambda\right|>0$ $u \lim _{n \rightarrow \infty} h_{n} / f_{n}=0$.

СЛЕДСТвИЕ 1.2. Пусть $a_{n} \neq 0$ при всех $n=1,2, \ldots$ и корни $\lambda_{n, 1} u \lambda_{n, 2}$ многочленов $z^{2}-b_{n} z-a_{n}$ таковь, что $\lim _{n \rightarrow \infty} \lambda_{n, 1}=\lambda u \underline{\lim }_{n \rightarrow \infty}\left|\lambda_{n, 2}\right|>|\lambda|$. Тогда существует единственное (с точностью до постоянного множителя) решение $\left\{h_{n}\right\}_{n=-1}^{\infty}$ разностного уравнения (160) такое, что существует предел $\lim _{n \rightarrow \infty} h_{n+1} / h_{n}=\lambda$. При этом для всякого другого решения $\left\{f_{n}\right\}_{n=-1}^{\infty}$ имеют место соотношения $\underline{\lim }_{n \rightarrow \infty}\left|f_{n+1} / f_{n}-\lambda\right|>0 u \lim _{n \rightarrow \infty} h_{n} /\left(f_{n+1}-\lambda f_{n}\right)=0$. 
СлЕДСТвИЕ 1.3. Пусть корни $\lambda_{n, 1} u \lambda_{n, 2}$ многочлена $z^{2}-b_{n} z-a_{n}$ таковы, чmo

$$
\varlimsup_{n \rightarrow \infty}\left|\frac{\lambda_{n, 1}}{\lambda_{n, 2}}\right|+2 \varlimsup_{n \rightarrow \infty}\left|\frac{\lambda_{n, 1}}{\lambda_{n, 2}}-\frac{\lambda_{n, 1}}{\lambda_{n-1,2}}\right|^{1 / 2}<1 .
$$

Тогда непрерьвная дробъ (159) сходится.

СлЕДСтвиЕ 1.4. Пусть непрерывная дробъ (159) такова, что

$$
\varlimsup_{n \rightarrow \infty}\left|b_{n}-1\right|+2 \varlimsup_{n \rightarrow \infty}\left|a_{n}+b_{n}-1\right|^{1 / 2}<1 .
$$

Тогда непрерывная дробъ (159) сходится.

Одним из следствий теоремы Перрона является существование единственного решения минимального роста, т. е. такого решения $\left\{h_{n}\right\}_{n=0}^{\infty}$ разностного уравнения (158), что для всякого другого (линейно с ним независимого) решения $\left\{f_{n}\right\}_{n=0}^{\infty}$ имеет место равенство $\lim _{n \rightarrow \infty} h_{n} / f_{n}=0$. Легко видеть, что для этого единственного (с точностью до постоянного множителя) решения имеет место равенство $\lim _{n \rightarrow \infty} h_{n+1} / h_{n}=\lambda$, где $\lambda$ - наименьший по модулю корень характеристического многочлена.

Как отмечалось, числители $\left\{P_{n}\right\}_{n=1}^{\infty}$ и знаменатели $\left\{Q_{n}\right\}_{n=1}^{\infty}$ непрерывной дроби (159) являются решениями (линейно независимыми в силу начальных условий) разностного уравнения (160). Так как любое решение разностного уравнения (160) и, в частности, решение $\left\{h_{n}\right\}_{n=1}^{\infty}$ минимального роста является линейной комбинацией линейно независимых решений $\left\{P_{n}\right\}_{n=1}^{\infty}$ и $\left\{Q_{n}\right\}_{n=1}^{\infty}$, то при всех $n=1,2, \ldots$ имеем равенство $h_{n}=C_{1} P_{n}+C_{2} Q_{n}$. Следовательно, если $C_{1}=0$, то

$$
\lim _{n \rightarrow \infty} \frac{P_{n}}{Q_{n}}=\lim _{n \rightarrow \infty} \frac{C_{2} P_{n}}{h_{n}}=\infty .
$$

Если $C_{1} \neq 0$, то

$$
\lim _{n \rightarrow \infty} \frac{h_{n}}{Q_{n}}=0=\lim _{n \rightarrow \infty} \frac{C_{1} P_{n}}{Q_{n}}+C_{2} .
$$

Это означает, что существует предел $\lim _{n \rightarrow \infty} P_{n} / Q_{n}=-C_{2} / C_{1}$, т. е. непрерывная дробь (159) сходится к значению $-C_{2} / C_{1}$. Приведенное рассуждение является основным в доказательстве одной из самых красивых теорем в теории непрерывных дробей - теоремы Ван Флека [190] о сходимости непрерывной дроби

$$
a_{0}+\frac{a_{1} z}{1+\frac{a_{2} z}{1+\ddots}}
$$

(такая непрерывная дробь называется правильной $C$-дробью) с предельно постоянными коэффициентами.

ТЕОРЕМА ВАН ФЛЕКА. Пусть коэффициенты правильной С-дроби (167) имеют предел $\lim _{n \rightarrow \infty} a_{n}=a \neq 0$. Тогда $C$-дробъ (167) сходится $к$ мероморфной функиии равномерно на компактах, лежащих в $\mathbb{C} \backslash \Gamma$, где $\Gamma=\{z \in \mathbb{C}$ : $z=-t /(4 a), t \geqslant 1\}$, и не содержащих полюсов предельной функции. 
Отметим, что разностное уравнение (160) применительно к непрерывной дроби (167) приобретает следующий вид:

$$
X_{n}=X_{n-1}+a_{n} z X_{n-2}, \quad n=1,2, \ldots,
$$

а множество тех $z \in \mathbb{C}$, для которых корни характеристического многочлена $\lambda^{2}-\lambda-a z$ разностного уравнения (168) равны по модулю, совпадает с указанным Ван Флеком разрезом $\Gamma$, на котором нет сходимости непрерывной дроби (167). Другими словами, при $z \in \Gamma$ нарушаются предположения теоремы Пуанкаре-Перрона. А. А. Гончар дополнил теорему Ван Флека следующим весьма важным замечанием, из которого следует, в частности, что область $\mathbb{C} \backslash \Gamma$ является максимальной областъю сходимости непрерывной дроби (167).

Теорема (дополнение Гончара к теореме Ван Флека). B предположениях теоремы Ван Флека функиия, к которой сходится непрерывная дробъ (167), не может быть мероморфной функиией ни в какой области $(\mathbb{C} \backslash \Gamma) \cup\left\{\left|z-z_{0}\right|<\varepsilon\right\}$, где $z_{0} \in \Gamma, \varepsilon>0$.

Теорема Ван Флека и дополнение Гончара к ней распространены в [191], [105] на случай $T$-дробей

$$
a_{0}+\frac{a_{1} z}{1+b_{1} z+\frac{a_{2} z}{1+b_{2} z+\ddots}}
$$

с предельно периодическими коэффициентами. Как известно, T-дроби сходятся в окрестностях точек $z=0$ и $z=\infty$ (в [192] указаны явные формулы для радиусов кругов сходимости произвольной $T$-дроби). Например, простейшая $T$-дробь $\frac{z \mid}{\mid 1-z}+\frac{z \mid}{\mid 1-z}+\cdots$ при $|z|<1$ сходится к функции $f_{1}(z)=z$, а при $|z|>1-$ к функции $f_{2}(z)=-1$. Каждая из этих функций допускает голоморфное продолжение на всю комплексную плоскость, но при этом $f_{1}(z) \not \equiv f_{2}(z)$. На этом примере видно, что мероморфная функция $f(z)$, к которой сходится $T$-дробь $\frac{z \mid}{\mid 1-z}+\frac{z \mid}{\mid 1-z}+\cdots$, не может быть однозначной мероморфной функцией ни в какой области вида $(\mathbb{C} \backslash\{|z|=1\}) \cup\left\{\left|z-z_{0}\right|<\varepsilon\right\}$, где $\left|z_{0}\right|=1$, $\varepsilon>0$. Аналогичное утверждение справедливо и для произвольных $T$-дробей с предельно периодическими коэффициентами. Точнее, имеет место следующая теорема [192].

Теорема 14. Пусть $m \in \mathbb{N}$, и пусть коэфбициенты Т-дроби (169) имеют периодические предель $\lim _{n \rightarrow \infty} a_{n m+l}=a^{l} \neq 0, \lim _{n \rightarrow \infty} b_{n m+l}=b^{l}, l=$ $1, \ldots, m$. Тогда Т-дробъ (169) сходится $\kappa$ мероморфной функции $f(z)$ равномерно на компактах, лежащих в $\mathbb{C} \backslash(\Gamma \cup K)$ и не содержащих полюсов функции $f(z)$, где $K$ - конечное множество, содержащее не более $m(m-1)$ точек (не более $[m(m-1) / 2]$ точек, если $\left.b^{1}=\cdots=b^{m}=0\right)$,

$$
\Gamma=\left\{z \in \mathbb{C}: z^{-m} \operatorname{Tr}^{2}\left(\left(\begin{array}{cc}
0 & a^{1} z \\
1 & 1+b^{1} z
\end{array}\right) \times \cdots \times\left(\begin{array}{cc}
0 & a^{m} z \\
1 & 1+b^{m} z
\end{array}\right)\right) \in E\right\}
$$


E - отрезок $\left[0,4(-1)^{m} a^{1} \cdots a^{m}\right], \operatorname{Tr}(\cdot)$ - след матрищы, стоящей внутри скобок. При этом функция $f(z)$ мероморфно продолжается в $\mathbb{C} \backslash \Gamma$ и не может быть однозначной мероморфной функиией ни в какой области $(\mathbb{C} \backslash \Gamma) \cup$ $\left\{\left|z-z_{0}\right|<\varepsilon\right\}$, где $z_{0} \in \Gamma, \varepsilon>0$.

При $m=1$ множество $K$ пусто. Для $C$-дробей множество $K$ пусто и при $m=2$. На непустоте множества $K$ при $m>2$ и фактическом отсутствии сходимости в точках множества $K$ строится контрпример к известной Паде-гипотезе (см. функцию (108), указанную в главе 1, п. 2.3, заданную непрерывной дробью (109)).

В основе доказательства сходимости T-дроби (169) с предельно периодическими коэффициентами вне множества $\Gamma \cup K$ лежит теорема Пуанкаре-Перрона, распространенная на случай разностных уравнений с предельно периодическими коэффициентами. В основе доказательства утверждения, аналогичного дополнению Гончара к теореме Ван Флека, лежат известные формулы, выражающие коэффициенты $T$-дроби через ганкелевы определители предельной функции (см. [9]), а также двухточечный аналог теоремы Пойа [193] об оценке сверху ганкелевых определителей мероморфной функции посредством емкости множества ее особенностей.

ТЕОРема (двухточечный аналог теоремы Пойа, см. [194]). Пусть $E$ - замкнутое множество комплексной плоскости, не содержащее точек 0 u $\infty$, $f(z)$ - функиия, (кусочно) мероморфная в компонентах $\mathbb{C} \backslash E$, содержащих точки 0 и $\infty$, и пусть $f(z)=\sum_{n=n_{1}}^{\infty} \alpha_{n} z^{n}, f(z)=\sum_{-\infty}^{n_{2}} \beta_{n} z^{n}\left(n_{1}, n_{2} \in \mathbb{Z}\right)$ - разложения функиии $f(z)$ в ряд Лорана в окрестностях точек 0 u $\infty$ соответственно. Тогда

¿əe

$$
\varlimsup_{n \rightarrow \infty}\left|H_{n}\right|^{1 / n^{2}} \leqslant \sqrt{d(E) d\left(E^{-1}\right)} e^{-\gamma(E)},
$$

$$
H_{n}=\left|\begin{array}{ccc}
\left(\alpha_{n}-\beta_{n}\right) & \ldots & \left(\alpha_{0}-\beta_{0}\right) \\
\ldots \ldots \ldots \ldots \ldots \ldots \ldots & \ldots \ldots \ldots \\
\left(\alpha_{0}-\beta_{0}\right) & \ldots & \left(\alpha_{-n}-\beta_{-n}\right)
\end{array}\right|
$$

$d(E)$ и $d\left(E^{-1}\right)$ - емкости компактов $E$ u $E^{-1}=\left\{z \in \mathbb{C}: z^{-1} \in E\right\}, \gamma(E)=0$, если точки 0 и лежат в разных компонентах дополнения $\mathbb{C} \backslash E, u \gamma(E)=$ $g(0, \infty)$ в противном случае, где $g(z, \xi)-$ функиия Грина компоненты $\mathbb{C} \backslash E$, содержащей точки 0 и $\infty$.

Теорема, аналогичная теореме Ван Флека и дополнению Гончара к ней, справедлива не только для $T$-дробей (169), но и для других видов предельно периодических непрерывных дробей, в частности, для общих (не обязательно правильных) $C$-дробей

$$
\frac{a_{1} z^{\alpha_{1}}}{1+\frac{a_{2} z^{\alpha_{2}}}{1+\cdot}}
$$

(подробнее см. работу [193]). Доказательство соответствующей теоремы проводится по той же вышеописанной схеме. Сначала выводятся явные формулы для ганкелевых определителей меромофной функции, к которой схо- 
дится $C$-дробь (170). А затем применяется усиленный двухточечный аналог теоремы Пойа, в котором учитывается разный порядок касания подходящих дробей $C$-дроби (170) с предельной мероморфной функцией в точках $z=0$ и $z=\infty$. Более того, имея в наличии формулы для ганкелевых определителей и используя для доказательства сходимости $C$-дроби (170) не теорему Пуанкаре-Перрона, а известный критерий Ворпицкого сходимости непрерывных дробей [195], по этой же схеме можно получить некоторые новые утверждения, связанные с гипотезой Лейтона.

\section{2. Гипотеза Лейтона}

Хорошо известный критерий Ворпицкого [195] является, по-видимому, первым критерием сходимости числовых непрерывных дробей с комплексными коэффициентами.

КРИтЕРий ВоРПИцКОГО. Непрерывная дробъ $\frac{a_{1}}{1+\frac{a_{2}}{1+\cdot}}$ cходится, если
$\left|a_{n}\right| \leqslant 1 / 4$ при всех достаточно больших $n \geqslant n_{0}$.

Так как $|z|^{n} \leqslant 1 / 4$ при всех $|z|<1$ и достаточно больших $n \geqslant n(z)$, то из критерия Ворпицкого следует, что непрерывная дробь Рамануджана

$$
R(z)=1+\frac{z}{1+\frac{z^{2}}{1+\frac{z^{3}}{1+\ddots}}}
$$

сходится в единичном круге $D=\{|z|<1\}$. При этом нетрудно показать, что предельная функция $R$ мероморфна в $D$. Из результатов Рамануджана следует, что единичный круг $D$ является естественной областью существования функции $R$ (это замечание содержится в монографии Уолла [7]).

В 1940 г. Лейтон (W. Leighton) высказал гипотезу, что тем же свойством (непродолжимости за пределы круга $D$ ) обладает всякая функция $f$, представимая $C$-дробью (170) такой, что $\lim _{k \rightarrow \infty} \alpha_{k}=\infty$ и на коэффициенты $a_{k}$ наложены определенные естественные условия.

ГиПотезА ЛЕЙтонА. Пустъ $C$-дробъ (170) такова, что $\alpha_{n} \in \mathbb{N}, a_{n} \in \mathbb{C} \backslash\{0\}$ $u$

$$
\lim _{n \rightarrow \infty} \alpha_{n}=\infty, \quad \lim _{n \rightarrow \infty}\left|a_{n}\right|^{1 / \alpha_{n}}=1 .
$$

Тогда C-дробъ (170) сходится в круге $D=\{|z|<1\} \kappa$ функиии $f$, мероморфной в $D$, и окружность $|z|=1$ является естественной границей мероморфности функции $f$.

Как и для непрерывной дроби Рамануджана, первая часть гипотезы Лейтона следует из критерия Ворпицкого. Основное содержание гипотезы заключено в утверждении о непродолжимости мероморфной функции $f$ за пределы круга $D$.

Скотт и Уолл [196] доказали гипотезу Лейтона для частного случая $\alpha_{n}=m^{n}$, $a_{n}=a, n=1,2, \ldots$. 
Из результатов Трона и ряда других авторов следует, что гипотеза Лейтона верна для показателей $\alpha_{n}$ таких, что $\lim _{\rightarrow \infty} \alpha_{n} / \alpha_{n-1} \rightarrow \infty$ (т. е. для очень быстро возрастающих показателей).

А. А. Гончар значительно усилил вышеприведенные результаты и показал, что гипотеза Лейтона верна для неубывающих показателей $\alpha_{n}$. Точнее, он доказал следующую теорему [197].

ТеОРема ГОНчара. Пусть С-дробъ (170) такова, что выполнены предположения (171) гипотезы Лейтона, а также при всех $n=1,2, \ldots$ выполняется условие

$$
\alpha_{n}-\alpha_{n-1}+\alpha_{n-2}-\alpha_{n-3}+\cdots+(-1)^{n-1} \alpha_{1} \geqslant 0 .
$$

Тогда имеет место утверждение Лейтона.

Очевидно, что для неубывающей последовательности натуральных показателей $\left\{\alpha_{n}\right\}_{n=1}^{\infty}$ условие $(172)$ выполняется.

В [193] показано, что, сохраняя условия (172), в теореме Гончара условия (171) можно заменить чуть более слабыми условиями

$$
\lim _{n \rightarrow \infty} \frac{\alpha_{1}+\cdots+\alpha_{n}}{n}=\infty \quad \text { и } \quad \lim _{n \rightarrow \infty}\left|4 a_{n}\right|^{1 / \alpha_{n}}=1 .
$$

Заметим, что условие (172) в теореме Гончара фактически означает отсутствие степенного ряда $f^{\infty}$, соответствующего $C$-дроби (170) в окрестности точки $z=\infty$. Если отказаться от условия (172), то такой ряд возникает, однако его существование не гарантирует сходимости к нему $C$-дроби (170) в окрестности точки $z=\infty$. Такую сходимость можно получить, используя некоторые дополнительные соображения. Например, имеет место следующая теорема [193].

ТЕОРема 15. Пусть непрерывная дробъ

$$
\frac{a_{1} z^{\alpha_{1}}}{1+b_{1} z^{\alpha_{1}}+\frac{a_{2} z^{\alpha_{2}}}{1+b_{2} z^{\alpha_{2}}+\cdot \cdot}}
$$

такова, что

$$
\lim _{n \rightarrow \infty} \alpha_{n}=\infty, \quad \lim _{n \rightarrow \infty}\left|a_{n}\right|^{1 / \alpha_{n}}=\left|b_{n}\right|^{1 / \alpha_{n}}=1, \quad \varlimsup_{n \rightarrow \infty}\left|\frac{a_{n+1}}{b_{n+1}}\right|^{1 / \alpha_{n}} \leqslant 1 .
$$

Тогда имеют место следующие утверждения.

$1^{\circ}$. Непрерьвная дробъ (174) сходится $\kappa$ (кусочно) мероморфной функции $f$ равномерно в сферической метрике на компактах, лежащих в открытом множестве $\{|z|<1\} \cup\{|z|>1\}$.

$2^{\circ}$. Функция $f$ не может быть мероморфной функиией ни в какой области вида $\{|z|<1\} \cup\{|z|>1\} \cup\left\{\left|z-z_{0}\right|<\varepsilon\right\}$, где $\left|z_{0}\right|=1, \varepsilon>0$.

\section{3. Сходимость непрерывной дроби Роджерса-Рамануджана}

Непрерывной дробью Роджерса-Рамануджана называется следующая правильная $C$-дробь:

$$
1+\frac{q z}{1+\frac{q^{2} z}{1+\ddots}}
$$


где $q \neq 0$ - произвольный комплексный параметр. Случай $|q|>1$ интереса не представляет, так как при таких $q$ непрерывная дробь Роджерса-Рамануджана сходится лишь при $z=0$.

Из критерия Ворпицкого следует, что непрерывная дробь (176) сходится при всех $z \in \mathbb{C}$, если $|q|<1$, и сходится при всех $|z| \leqslant 1 / 4$, если $|q|=1$. Функция, к которой сходится непрерывная дробь (176), найдена Роджерсом и Рамануджаном при помощи следующего рассуждения. Для функции Роджерса-Рамануджана

$$
G_{q}(z)=\sum_{k=0}^{\infty} z^{k} q^{k^{2}} \frac{1}{(q)_{k}}
$$

где $(q)_{0}=1,(q)_{n}=(1-q) \cdots\left(1-q^{n}\right)$, непосредственным образом проверяется функциональное равенство

$$
G_{q}(z)=G_{q}(q z)+q z G_{q}\left(q^{2} z\right)
$$

из которого для функции $H_{q}(z)=\frac{G_{q}(z)}{G_{q}(q z)}$ следует равенство

$$
H_{q}(z)=1+\frac{q z}{H_{q}(q z)}
$$

Из этого же равенства для $H_{q}(q z), H_{q}\left(q^{2} z\right)$ и т. д. следует бесконечная цепочка равенств

$$
H_{q}(z)=1+\frac{q z}{H_{q}(q z)}=1+\frac{q z}{1+\frac{q^{2} z}{H_{q}\left(q^{2} z\right)}}=1+\frac{q z}{1+\frac{q^{2} z}{1+\frac{q^{3} z}{H_{q}\left(q^{3} z\right)}}}=\cdots,
$$

которая влечет за собой (после некоторых стандартных рассуждений) равенство

$$
H_{q}(z)=1+\frac{q z}{1+\frac{q^{2} z}{1+\frac{q^{3} z}{1+\ddots}}} .
$$

Другое доказательство равенства (179) дал М.Д. Хиршхорн, основываясь на полученных им явных формулах для числителя $P_{q, n}(z)$ и знаменателя $Q_{q, n}(z) n$-й подходящей дроби (176). А именно, Хиршхорн [198] показал, что

$$
\begin{aligned}
P_{q, n}(z) & =\sum_{k=0}^{[(n+1) / 2]} z^{k} q^{k^{2}} \frac{(q)_{n+1-k}}{(q)_{k}(q)_{n+1-2 k}} \\
Q_{q, n}(z) & =P_{q, n-1}(q z) .
\end{aligned}
$$

Так как при $|q|<1$

$$
\lim _{n \rightarrow \infty} \frac{(q)_{n+1-k}}{(q)_{n+1-2 k}}=\lim _{n \rightarrow \infty}\left(1-q^{n+2-2 k}\right) \cdots\left(1-q^{n+1-k}\right)=1,
$$


то из формул Хиршхорна следует, что при $|q|<1, z \in \mathbb{C}$

$$
\begin{aligned}
& \lim _{n \rightarrow \infty} P_{q, n}(z)=\sum_{k=0}^{\infty} z^{k} q^{k^{2}} \frac{1}{(q)_{k}}=G_{q}(z), \\
& \lim _{n \rightarrow \infty} Q_{q, n}(z)=G_{q}(q z), \\
& \lim _{n \rightarrow \infty} \frac{P_{q, n}}{Q_{q, n}}(z)=\frac{G_{q}(z)}{G_{q}(q z)}=H_{q}(z) .
\end{aligned}
$$

Формулы Хиршхорна оказались весьма полезными и при изучении наиболее интересного случая $|q|=1$. Случай $q=\exp (2 \pi i \tau)$, где $\tau$ - рациональное число, легко исследуется, так как в этом случае непрерывная дробь (176) является периодической непрерывной дробью. Поэтому далее будем предполагать, что $q=\exp (2 \pi i \tau)$, где $\tau-$ иррациональное число. Обозначим через $\Lambda^{\beta}$ последовательность натуральных чисел такую, что $\lim _{n \in \Lambda^{\beta}} q^{n}=\beta,|\beta|=1$. Тогда по аналогии с равенством (180) имеем равенство

$\lim _{n \in \Lambda^{\beta}} \frac{(q)_{n+1-k}}{(q)_{n+1-2 k}}=\lim _{n \in \Lambda^{\beta}}\left(1-q^{n+2-2 k}\right) \cdots\left(1-q^{n+1-k}\right)=\left(1-\beta q^{2-2 k}\right) \cdots\left(1-\beta q^{1-k}\right)$.

Опираясь на это равенство и формулы Хиршхорна, Д. Любински [107] показал, что равномерно на компактах, лежащих в круге $\left\{|z|<R_{q}\right\}$, где $R_{q}$ - радиус голоморфности функции $G_{q}$, имеют место предельные равенства

$$
\begin{aligned}
& \lim _{n \in \Lambda^{\beta}} P_{q, n}(z)=G_{q}(z) \overline{G_{q}(\overline{\beta q z})}, \\
& \lim _{n \in \Lambda^{\beta}} Q_{q, n}(z)=G_{q}(q z) \overline{G_{q}(\overline{\beta q z})} .
\end{aligned}
$$

Эти равенства лежат в основе доказательства следующей теоремы [107].

ТЕОРемА ЛЮБИНСКИ. Для всякого $q=\exp (2 \pi i \tau)$, где $\tau$ - иррациональное число, непрерьвная дробъ (176) Роджерса-Рамануджана сходится к функиии $H_{q}$ равномерно на компактах, лежащих в $\left\{|z|<R_{q}\right\} \backslash \Omega_{q}$, где $\Omega_{q}$ - обгединение окружностей с центром в нуле, проходящих через полюсы функиии $H_{q}$. При этом соответствующее утверждение становится неверным, если множество $\Omega_{q}$ заменить на любое меньшее замкнутое множество.

На равенствах (181)-(182) основан, как отмечалось в главе 1, п. 2.3, контрпример Любински к Паде-гипотезе.

Из явного вида (177) функции $G_{q}$ и формулы Адамара следует, что радиус $R_{q}$ голоморфности функции $G_{q}$ при $q=\exp (2 \pi i \tau), \tau$ - иррационально, равен $\underline{\lim }_{n \rightarrow \infty}\left|(q)_{n}\right|^{1 / n}$. Г. Харди и Дж. Э. Литтлвуд [199] доказали равенство $R_{q}=\underline{\lim }_{n \rightarrow \infty}\left|1-q^{n}\right|^{1 / n}$. Д. Любински [107] показал, что радиус голоморфности $R_{q}$ при почти всех $|q|=1$ принимает значение, равное 1 . Д. Любински [200] и Д. Петруска [201] доказали, что $R_{q}$ может принимать любое наперед заданное значение из отрезка $[0,1]$.

Любопытно, что радиус голоморфности $R_{q}$ функции $G_{q}$ и радиус мероморфности $\rho_{q}$ функции $H_{q}(z)=G_{q}(z) / G_{q}(q z)$ могут не совпадать, несмотря на тот 
факт, что окружность $|z|=R_{q}$ является естественной границей голоморфности функции $G_{q}$ (этот факт нетрудно получить из функционального уравнения (178) для функции $G_{q}$ ). После работы Д. Любински [107] остался открытым им же и отмеченный вопрос о возможности мероморфного продолжения функции $H_{q}$ за пределы круга $|z|<R_{q}$ при тех $q$, при которых $R_{q}<1$. Если $R_{q}=1$, то из явного вида ганкелевых определителей непрерывной дроби Роджерса-Рамануджана и теоремы Пойа следует, что такое продолжение невозможно. Если $R_{q}<1 / 4$, то такое мероморфное продолжение гарантируется критерием Ворпицкого по крайней мере в круг $|z|<1 / 4$. Используя вместо критерия Ворпицкого фундаментальные неравенства (см., например, [7]), Д. Любински показал, что функция $H_{q}$ имеет мероморфное продолжение в круг $|z|<1 /(2+|1+q|)$. Ответ на вопрос о мероморфном продолжении функции $H_{q}$ в круг $|z|<1$ при всех $q=\exp (2 \pi i \tau)$, где $\tau$ - иррационально, дается в следующей теореме Буслаева (см. [202]), уточняющей теорему Любински.

Теорема 16. Пусть $q=\exp (2 \pi i \tau)$, где $\tau-$ иррациональное число. Тогда справедливы следующие утверждения.

$1^{\circ}$. Функиия $H_{q}$ мероморфна в круге $|z|<1$. Окружность $|z|=1$ является естественной гранищей мероморфности функиии $H_{q}$.

$2^{\circ}$. Непрерывная дробъ (176) Роджерса-Рамануджана сходится $\kappa$ функции $H_{q}$ равномерно на компактах, лежащих в $\{|z|<1\} \backslash \Omega_{q}$, где $\Omega_{q}$ - объединение окружностей с центром в нуле, проходящих через полюсы функиии $H_{q}$. При этом соответствующее утверждение становится неверным, если множество $\Omega_{q}$ заменить на любое меньшее замкнутое множество.

\section{4. Некоторые приложения уточненной теоремы Пуанкаре}

В [171] получен ряд уточнений теоремы Пуанкаре. Первое из этих уточнений состоит в том, что определенное нетривиальное содержание теоремы Пуанкаре удается сохранить и в случае отказа от условия различности по модулю корней характеристического многочлена.

Уточнение 1. Пусть имеют место все предположения теоремы Пуанкаре, за исключением предположения о различности по модулю корней характеристического многочлена. Тогда либо $f_{n}=0$ при всех $n \geqslant n_{0}$, либо $\varlimsup_{n \rightarrow \infty}\left|f_{n}\right|^{1 / n}$ равен модулю одного из корней характеристического многочлена и существует предел

$$
\lim _{n \rightarrow \infty} \frac{f_{n}+\beta_{1} f_{n-1}+\cdots+\beta_{l} f_{n-l}}{\left|f_{n-1}\right|+\cdots+\left|f_{n-l}\right|}=0
$$

где $\beta_{1}, \ldots, \beta_{l}-$ коэффициенты многочлена $z^{l}+\beta_{1} z^{l-1}+\cdots+\beta_{l}=\prod_{j=1}^{l}\left(z-\lambda_{j}\right)$, $\lambda_{1}, \ldots, \lambda_{l}-$ корни характеристического многочлена, равные по модулю $\overline{\lim }_{n \rightarrow \infty}\left|f_{n}\right|^{1 / n}$.

Если все корни характеристического многочлена различны по модулю, то в этом случае число $l$ не может быть строго больше 1 и, следовательно, $l=1$ 
и равенство (183) означает, что существует предел $\lim _{n \rightarrow \infty} f_{n} / f_{n-1}=-\beta_{1}$, где $-\beta_{1}$ совпадает с одним из корней характеристического многочлена.

Следующее уточнение распространяет теорему Пуанкаре на случай рекуррентных соотношений неограниченного порядка.

Уточнение 2. Пусть элементы последователъности $\left\{f_{n}\right\}_{n=0}^{\infty}$ удовлетворяют рекуррентным соотношениям

$$
f_{n}+\alpha_{1, n} f_{n-1}+\cdots+\alpha_{n, n} f_{0}=0, \quad n=n_{0}, n_{0}+1, \ldots,
$$

с коэффициентами $\alpha_{1, n}, \ldots, \alpha_{n, n}$ такими, что многочленъ $1+\alpha_{1, n} z+$ $\cdots+\alpha_{n, n} z^{n}$ равномерно на компактах, лежащих в круге $|z|<R$, сходятся при $n \rightarrow \infty \kappa$ функици $\alpha(z) \not \equiv 0$. Тогда либо $\varlimsup_{n \rightarrow \infty}\left|f_{n}\right|^{1 / n} \leqslant R^{-1}$, либо $\left(\overline{\lim }_{n \rightarrow \infty}\left|f_{n}\right|^{1 / n}\right)^{-1}$ равен модулю одного из корней функиии $\alpha(z)$ и существуem nредел

$$
\lim _{n \rightarrow \infty} \frac{\left[f(z) \prod_{j=1}^{l}\left(1-z / \lambda_{j}\right)\right]_{n}}{\left|f_{n-1}\right|+\cdots+\left|f_{n-l}\right|}=0,
$$

где $\lambda_{1}, \ldots, \lambda_{l}-$ корни функции $\alpha(z)$, равные по модулю $\left(\overline{\lim }_{n \rightarrow \infty}\left|f_{n}\right|^{1 / n}\right)^{-1}$, a символом $[\cdot]_{n}$ обозначается коэффициент при $z^{n}$ степенного ряда, стоящего в квадратных скобках.

Например, если $\alpha(z)=1+\sum_{k=1}^{\infty} \alpha_{k} z^{k}-$ целая функция, нули которой различны по модулю, и последовательность $\left\{f_{n}\right\}_{n=0}^{\infty}$ удовлетворяет рекуррентным соотношениям

$$
f_{n}+\alpha_{1} f_{n-1}+\cdots+\alpha_{n} f_{0}=0, \quad n=n_{0}, n_{0}+1, \ldots,
$$

то из уточнения 2 следует, что либо $\varlimsup_{n \rightarrow \infty}\left|f_{n}\right|^{1 / n}=0$, либо существует предел $\lim _{n \rightarrow \infty} f_{n-1} / f_{n}$ и этот предел равен одному из нулей функции $\alpha(z)$.

Уточнения 1 и 2 теоремы Пуанкаре являются частными случаями (в которых сохраняется понятие рекуррентности соотношений) следующей теоремы.

Теорема 17. Пусть элементы последовательности $\left\{f_{n}\right\}_{n=-\infty}^{\infty}$ таковы, что $\overline{\lim }_{n \rightarrow \infty}\left|f_{-n}\right|^{1 / n} \leqslant \overline{\lim }_{n \rightarrow \infty}\left|f_{n}\right|^{1 / n}=1$ и при некотором положительном $\delta$

$$
\lim _{n \rightarrow \infty} e^{\delta n} \sum_{p=-\infty}^{\infty} f_{n-p} \alpha_{p, n}=0
$$

где $\alpha_{n}(z)=\sum_{p=-\infty}^{\infty} \alpha_{p, n} z^{p}-\oint у н к ц и и$, голоморфные в некоторой (не зависящей от $n$ ) окрестности окружности $|z|=1$ и равномерно сходящиеся в этой окрестности при $n \rightarrow \infty \kappa$ функиии $\alpha(z) \not \equiv$ 0. Тогда функция $\alpha(z)$ имеет на окружности $|z|=1$ по менъшей мере один нуль и существует предел (184), где $\lambda_{1}, \ldots, \lambda_{l}$ - корни функции $\alpha(z)$, лежащие на окружности $|z|=1$.

Заметим, что условие (185) выполняется при любом положительном $\delta$, если при всех $n=n_{0}, n_{0}+1, \ldots$ имеют место соотношения

$$
\sum_{p=-\infty}^{\infty} f_{n-p} \alpha_{p, n}=0
$$


При помощи теоремы 17 может быть доказан аналог теоремы Фабри об отношении для ортогональных рядов [203].

ТеОрема 18. Пусть $T_{n}(z), n=0,1, \ldots,-$ последовательность ортонормированных на отрезке $[-1,1]$ многочленов, построенных по удовлетворяющей условию Сегё

$$
\int_{-1}^{1} \frac{\ln \sigma^{\prime}(x)}{\sqrt{1-x^{2}}} d x>-\infty
$$

неубывающей на $[-1,1]$ функиии $\sigma(x)$, и пусть коэбфициенть ряда

$$
\sum_{n=0}^{\infty} F_{n} T_{n}(z)
$$

таковы, что существует предел $\lim _{n \rightarrow \infty} F_{n} / F_{n+1}=\lambda,|\lambda|>1$. Тогда ряд сходится равномерно внутри эллипса с фокусами в точках \pm 1 , проходящего через точку $\frac{\lambda+\lambda^{-1}}{2}, u \frac{\lambda+\lambda^{-1}}{2}$ - особая точка функции $F(z)=\sum_{n=0}^{\infty} F_{n} T_{n}(z)$, лежащая на гранище эллипса сходимости ряда (187).

Как и в теореме Фабри, первая часть теоремы о сходимости ряда (187) является простым следствием формулы Коши-Адамара для эллипса сходимости ортогонального ряда, а основное содержание теоремы сосредоточено в утверждении о том, что точка $\left(\lambda+\lambda^{-1}\right) / 2$ является особой точкой функции $F(z)$. Это утверждение доказывается путем сведения (при помощи теоремы 17) к классической теореме Фабри.

Как отмечалось в главе 1 , полюс $\xi_{n, 1}$ первой строки таблицы классических аппроксимаций Паде ряда $f(z)=\sum_{n=0}^{\infty} f_{n} z^{n}$ совпадает с отношением $f_{n} / f_{n+1}$. Исходя из этого замечания, А. А. Гончар высказал гипотезу о том, что утверждение, аналогичное теореме Фабри об отношении, имеет место не только для первой строки таблицы классических аппроксимаций Паде, но и для $m$-й строки таблицы как классических, так и обобщенных аппроксимаций Паде (многоточечных аппроксимаций Паде, аппроксимаций Паде ортогональных разложений, аппроксимаций Паде-Фабера). Напомним, например, определение ортогональных аппроксимаций Паде.

Ортогональной аппроксимаций Паде типа $(n, m)$ ряда $(187)$ по ортонормированным многочленам $T_{n}(z), n=0,1, \ldots$, называется рациональная функция $[n / m]_{F}=P_{n, m} / Q_{n, m}$, удовлетворяющая условиям $\operatorname{deg} P_{n, m} \leqslant n, \operatorname{deg} Q_{n, m} \leqslant m$, $Q_{n, m} \not \equiv 0$ и такая, что

$$
\left(Q_{n, m} F-P_{n, m}\right)(z)=\sum_{\nu=n+m+1}^{\infty} A_{\nu} T_{\nu}
$$

Из этого определения следует, что полюс $\tau_{n, 1}$ первой строки таблицы аппроксимаций Паде ортогональных разложений определяется из равенства

$$
\int_{-1}^{1}\left(x-\tau_{n, 1}\right) F(x) T_{n+1}(x) d \sigma(x)=0 .
$$


Если функция $\sigma(x)$ удовлетворяет условию Сегё (186), то коэффициенты хорошо известных трехчленных соотношений

$$
x T_{n}(x)=A_{n} T_{n+1}(x)+B_{n} T_{n}(x)+C_{n} T_{n-1}(x)
$$

имеют пределы

$$
\lim _{n \rightarrow \infty} A_{n}=\lim _{n \rightarrow \infty} C_{n}=\frac{1}{2}, \quad \lim _{n \rightarrow \infty} B_{n}=0
$$

Отсюда и из равенства (188) следует, что равенство $\lim _{n \rightarrow \infty} F_{n} / F_{n+1}=\lambda$, $|\lambda|>1$, эквивалентно равенству $\lim _{n \rightarrow \infty} \tau_{n, 1}=\left(\lambda+\lambda^{-1}\right) / 2$. Следовательно, при $m=1$ гипотеза Гончара для ортогональных разложений совпадает с теоремой 18. При $m>1$ гипотеза Гончара для случая классических аппроксимаций Паде доказана Суетиным (см. введение, п. 2.2, теорема Суетина), а для случая ортогональных разложений - Буслаевым [171].

ТЕОрема 19. Пусть неубивающая на отрезке $E=[-1,1]$ функиия $\sigma$ удовлетворяет условию Сегё (186), F- - ункиия, голоморфная в некоторой окрестности $E, \tau_{n, 1}, \ldots, \tau_{n, m}$ - полюсы $m$-й строки ортогональных аппроксимаицй Паде, и пусть $\lim _{n \rightarrow \infty} \tau_{n, j}=\tau_{j}(j=1, \ldots, m)$. Тогда справедливы следующие утверждения.

$1^{\circ} . \tau_{j} \in \mathbb{C} \backslash E, j=1, \ldots, m$.

$2^{\circ}$. Функиия $F$ мероморфно продолжается в эллипсы $D_{j}$ с фокусами в точках \pm 1, проходящие через точки $\tau_{j}(j=1, \ldots, m)$, и в максимальном из этих эллипсов имеет не более $m-1$ полюсов.

$3^{\circ}$. Все точки $\tau_{1}, \ldots, \tau_{m}$ являются особыми точками функции $F$, причем те из них, которые лежат строго внутри максимального из эллипсов $D_{1}, \ldots, D_{m}$, являются полюсами и других полюсов функиия $F$ в этом эллипсе не имеет.

Частный случай теоремы 19 был доказан Суетиным [204] при более слабом, по сравнению с условием Сегё, предположении $\sigma^{\prime}>0$ почти всюду на отрезке $[-1,1]$, но при дополнительном сильном предположении, что полюсы $\tau_{n, j}$ ортогональных аппроксимаций Паде сходятся со скоростью геометрической прогрессии.

В [171] доказана эквивалентность гипотез Гончара для различных видов обобщений аппроксимаций Паде.

Теорема 20. Пусть $F$ - функиия, голоморфная в окрестности содержащего более одной точки ограниченного континуума $E$ со свлзным дополнением, $\psi$ - функция, голоморфно и однолистно отображающая внешность континуума $E$ на внешность единичного круга, $\psi(\infty)=\infty, f(z)=F\left(\psi^{-1}(z)\right)$. Тогда следующие утверждения эквивалентны между собой.

$1^{\circ}$. Полюсы $m$-й строки таблицы классических аппроксимаций Паде регулярной части функции $f$ стремятся $к$ пределам $\lambda_{1}, \ldots, \lambda_{m}$.

$2^{\circ}$. Полюсы $m$-й строки табличь ортогоналъных аппроксимаций Паде функции $F$ в предположении, что $E=[-1,1]$ и выполнено условие Сегё (186) на неубываюшую функцию $\sigma$, стремятся $к$ пределам $\tau_{1}, \ldots, \tau_{m}$. 
$3^{\circ}$. Полюсы $m$-й строки таблицы аппроксимаций Паде-Фабера функции $F$ стремятся $\kappa$ пределам $\tau_{1}, \ldots, \tau_{m}$.

$4^{\circ}$. Полюсы $m$-й строки таблицы многоточечных аппроксимаций Паде функции $F$, построенных по таблище точек интерполячии $\left\{z_{k, n}\right\}, n=1,2, \ldots$, $k=1, \ldots, n$, удовлетворяющей условию

$$
\lim _{n \rightarrow \infty} \frac{\prod_{k=1}^{n}\left(z-z_{k, n}\right)}{c^{n} \psi^{n}(z)}=\omega(z) \neq 0, \quad z \in \mathbb{C} \backslash E,
$$

стремятся $\kappa$ пределам $\tau_{1}, \ldots, \tau_{m}$.

При этом $\left|\lambda_{j}\right|>1, \tau_{j} \in \mathbb{C} \backslash E u \lambda_{j}=\psi\left(\tau_{j}\right), j=1, \ldots, m$.

Подробнее о многоточечных аппроксимациях Паде, аппроксимациях ПадеФабера и доказательстве теоремы 20, основанном на распространении теоремы 17 на случай систем соотношений, см. [171].

\section{Список литературы}

[1] G. Frobenius, "Ueber Relationen zwischen den Näherungsbruchen von Potenzreihen", Borchardt J., XC (1880), 1-17.

[2] H. Padé, "Sur la représentation approchée d'une fonction par des fractions rationnelles", Ann. Sci. École Norm. Sup. (3), IX, Suppl. (1892), 3-93; http://www. numdam.org/item?id=ASENS_1892_3_9__S3_0.

[3] П.Л. Чебышёв, "О непрерывных дробях", Ученые зап. Имп. акад. наук, III (1855), 636-664; Полное собрание сочинений, II, Изд-во АН СССР, М.-Л., 1948, 103-126; фр. пер.: P. Tchébycheff, "Sur les fractions continues", J. de Math. Pures et Appl. Sér. 2, 3 (1858), 289-323.

[4] А. А. Марков, "Два доказательства сходимости некоторых непрерывных дробей", Избранные труды по теории непрерывных дробей и теории функиий, наименее уклоняющихся от нуля, Гостехиздат, М., 1948, 106-119; пер. с фр.: A. Markoff, "Deux démonstrations de la convergence de certaines fractions continues", Acta Math., 19:1 (1895), 93-104.

[5] Т.И. Стилтьес, Исследования о непрерывных дробях, ОНТИ, Харьков-Киев, 1936; пер. с фр.: T. J. Stieltjes, "Recherches sur les fractions continues", Reprint of Ann. Fac. Sci. Toulouse Sci. Math. Sci. Phys. 8:4 (1894), J76-J122, Ann. Fac. Sci. Toulouse Math. (6), 4:3 (1995), J76-J122; http://www.numdam.org/ item?id=AFST_1995_6_4_3_J76_0; T.-J. Stieltjes, "Recherches sur les fractions continues", Reprint of Ann. Fac., Suite et fin, Ann. Fac. Sci. Toulouse Sci. Math. Sci. Phys. 9:1 (1895), A5-A47, Ann. Fac. Sci. Toulouse Math. (6), 4:4 (1995), A5-A47; http://www.numdam.org/item?id=AFST_1995_6_4_4_A5_0.

[6] O. Perron, Die Lehre von den Kettenbrüchen. Band II. Analytisch-funktionentheoretische Kettenbrüche, Teubner, Stuttgart, 1957, vi+316 pp.

[7] H.S. Wall, Analytic theory of continued fractions, Chelsea, New York, 1973.

[8] Дж. Бейкер, П. Грейвс-Моррис, Аппроксимачии Паде, Мир, М., 1986, 504 с.; пер. с англ.: G.A. Baker, Jr., P. Graves-Morris, Padé approximants. Part I. Basic theory, Encyclopedia Math. Appl., 13, Addison-Wesley, Reading, MA, 1981, $\mathrm{xx}+325$ pp.; G. A. Baker Jr., P. Graves-Morris, Padé approximants. Part II. Extensions and applications, Encyclopedia Math. Appl., 14, Addison-Wesley, Reading, MA, 1981, xviii+215 pp.

[9] У. Джоунс, В. Трон, Непреръьвные дроби, Мир, М., 1985, 416 с.; пер. с англ.: W. B. Jones, W.J. Thron, Continued fractions. Analytic theory and applications, Encyclopedia Math. Appl., 11., Addison-Wesley, Reading, MA, 1980, xxix+428 pp. 
[10] Н.И. Ахиезер, “Чебышевское направление в теории аппроксимаций”, Матемаmuка XIX века, Вып. 3, ред. А. Н. Колмогоров, А. П. Юшкевич, Наука, М., 1987, 9-79.

[11] Е. М. Никишин, В.Н. Сорокин, Рационалъные аппроксимачии и ортогоналъность, Наука, M., 1988, 256 с.; англ. пер.: E. M. Nikishin, V. N. Sorokin, Rational approximations and orthogonality, Transl. Math. Monogr., 92, Amer. Math. Soc., Providence, RI, 1991, viii+221 pp.

[12] А.А. Гончар, "Рациональные аппроксимации аналитических функций", Совр. пробл. матем., 1, МИАН, М., 2003, 83-106; англ. пер.: А. A. Gonchar, "Rational approximation of analytic functions", Proc. Steklov Inst. Math., 272, suppl. 2 (2011), $44-57$.

[13] W. Van Assche, "Padé and Hermite-Padé approximation and orthogonality", Surv. Approx. Theory, 2 (2006), 61-91; arXiv: math/0609094.

[14] A.-L. Cauchy, Cours d'analyse de l'École Royale Polytechnique. Première partie. Analyse algébrique, L'Imprimerie Royale, Paris, 1821; Instrumenta Rationis. Sources for the History of Logic in the Modern Age, VII, Cooperativa Libraria Universitaria Editrice Bologna, Bologna, 1992, clxviii+607 pp.; http://mathdoc.emath.fr/cgi-bin/ oeitem?id=OE_CAUCHY_2_3_P5_0.

[15] C. G. J. Jacobi, "Über die Darstellung einer Reihe gegebner Werthe durch eine gebrochne rationale Function", J. Reine Angew. Math., 30 (1846), 127-156.

[16] Ch. Hermite, "Sur la fonction exponentielle", C. R. Acad. Sci. Paris, 77 (1873), 18-24, 74-79, 226-233, 285-293.

[17] J. Hadamard, "Essai sur l'étude des fonctions données par leur développement de Taylor", J. Math. (4), VIII (1892), 101-186 (Thèse. Gauthier-Villars, Paris).

[18] R. de Montessus, "Sur les fractions continues algébriques", Bull. Soc. Math. France, 30 (1902), 28-36.

[19] А. А. Гончар, "О сходимости аппроксимаций Паде", Матем. сб., 92(134):1(9) (1973), 152-164; англ. пер.: А. A. Gončar, "On the convergence of Padé approximants", Math. USSR-Sb., 21:1 (1973), 155-166.

[20] А. А. Гончар, "Скорость рациональной аппроксимации и свойство однозначности аналитических функций в окрестности изолированной особой точки", $\mathrm{Ma}$ тем. сб., 94(136):2(6) (1974), 265-282; англ. пер.: А. А. Gončar, "The rate of rational approximation and the property of single-valuedness of an analytic function in the neighborhood of an isolated singular point", Math. USSR-Sb., 23:2 (1974), $254-270$.

[21] А. А. Гончар, "О сходимости аппроксимаций Паде для некоторых классов мероморфных функций”, Матем. сб., 97(139):4(8) (1975), 607-629; англ. пер.: A. A. Gončar, "On convergence of Padé approximants for some classes of meromorphic functions", Math. USSR-Sb., 26:4 (1975), 555-575.

[22] А.А. Гончар, "О сходимости обобщенных аппроксимаций Паде мероморфных функций”, Матем. сб., 98(140):4(12) (1975), 564-577; англ. пер.: А. А. Gončar, "On the convergence of generalized Padé approximants of meromorphic functions", Math. USSR-Sb., 27:4 (1975), 503-514.

[23] А.А. Гончар, "О скорости рациональной аппроксимации некоторых аналитических функций”, Матем. сб., 105(147):2 (1978), 147-163; англ. пер.: A. A. Gončar, "On the speed of rational approximation of some analytic functions", Math. USSR-Sb., 34:2 (1978), 131-145.

[24] А. А. Гончар, "5.6. Рациональная аппроксимация аналитических функций”, Исследования по линейным операторам и теории функций, 99 нерешенных задач линейного и комплексного анализа, Зап. научн. сем. ЛОМИ, 81, Наука, Ленинград. отд., Л., 1978, 182-185; англ. пер.: А.А. Gonchar, "5.6. Rational approximation of analytic functions", J. Soviet Math., 26:5 (1984), 2218-2220. 
[25] А. А. Гончар, Г. Лопес Лагомасино, "О теореме Маркова для многоточечных аппроксимаций Паде", Матем. сб., 105(147):4 (1978), 512-524; англ. пер.: A. A. Gonchar, G. López Lagomasino, "On Markov's theorem for multipoint Padé approximants", Math. USSR-Sb., 34:4 (1978), 449-459.

[26] А. А. Гончар, Е. А. Рахманов, "О сходимости совместных аппроксимаций Паде для систем функций марковского типа", Теория чисел, математический анализ и их приложения, Сборник статей. Посвящается академику Ивану Матвеевичу Виноградову к его девяностолетию, Тр. МИАН СССР, 157, 1981, 31-48; англ. пер.: А. A. Gonchar, E. A. Rakhmanov, "On convergence of simultaneous Padé approximants for systems of functions of Markov type", Proc. Steklov Inst. Math., 157 (1983), 31-50.

[27] А. А. Гончар, "Полюсы строк таблицы Паде и мероморфное продолжение функций", Матем. сб., 115(157):4(8) (1981), 590-613; англ. пер.: А. А. Gončar, "Poles of rows of the Padé table and meromorphic continuation of functions", Math. USSR-Sb., 43:4 (1982), 527-546.

[28] А.А. Гончар, "О равномерной сходимости диагональных аппроксимаций Паде", Матем. сб., 118(160):4(8) (1982), 535-556; англ. пер.: А. A. Gonchar, "On uniform convergence of diagonal Padé approximants", Math. USSR Sb., 46:4 (1983), 539-559.

[29] А. А. Гончар, "О сходимости диагональных аппроксимаций Паде в сферической метрике", Математические структуры, вычислительная математика, математическое моделирование, Труды, посвященные 70-летию академика Л. Илиева, София, 1984, 29-35.

[30] А. А. Гончар, "О скорости рациональной аппроксимации аналитических функций", Современные проблемы математики. Дифференииальные уравнения, математический анализ и их приложения, Сборник статей. Посвящается академику Льву Семеновичу Понтрягину к его семидесятипятилетию, Тр. МИАН CCCP, 166, 1984, 52-60; англ. пер.: А. A. Gonchar, "On the degree of rational approximation of analytic functions", Proc. Steklov Inst. Math., 166 (1986), 53-61.

[31] A. A. Gonchar, "Rational approximation of analytic functions", Linear and complex analysis problems book, Lecture Notes in Math., 1043, Springer-Verlag, Berlin, 1984, 471-474.

[32] J. Nuttall, "The convergence of Padé approximants of meromorfic functions", J. Math. Anal. Appl., 31 (1970), 147-153.

[33] J. Nuttall, "The convergence of certain Padé approximants", Proceedings of the International Conference of Padé Approximants, Continued Fractions and Related Topics (Univ. Colorado, Boulder, CO, 1972), Rocky Mountain J. Math., 4 (1974), 269-272.

[34] J. L. Gammel, J. Nuttall, "Convergence of Padé approximants to quasianalytic functions beyond natural boundaries", J. Math. Anal. Appl., 43 (1973), 694-696.

[35] J. Nuttall, "The convergence of Padé approximants to functions with branch points", Padé and rational approximation, Proc. Internat. Sympos. (University of South Florida, Tampa, FL, 1976), Academic Press, New York, 1977, 101-109.

[36] J. Nuttall, R. S. Singh, "Orthogonal polynomials and Padé approximants associated with a system of arcs", J. Approx. Theory, 21 (1977), 1-42.

[37] J. Nuttall, "Sets of minimal capacity Padé approximants and the bubble problem", Bifurcation phenomena in mathematical physics and related topics, eds. C. Bardos and D. Bessis, Reidel, Dordrecht, 1980, 185-201.

[38] J. Nuttall, "Hermite-Padé approximants to functions meromorphic on a Riemann surface", J. Approx. Theory, 32:3 (1981), 233-240.

[39] J. Nuttall, "The convergence of Padé approximants and their generalizations", The Riemann problem, complete integrability and arithmetic applications (Bures-sur- 
Yvette, New York, 1979-1980), Lecture Notes in Math., 925, Springer, Berlin-New York, 1982, 246-257.

[40] J. Nuttall, "Asymptotics of diagonal Hermite-Padé polynomials", J. Approx. Theory, 42:4 (1984), 299-386.

[41] D. Damanik, R. Killip, B. Simon, "Necessary and sufficient conditions in the spectral theory of Jacobi matrices and Schrödinger operators", Int. Math. Res. Not., 2004, № 22, 1087-1097.

[42] B. Simon, A. Zlatoš, "Sum rules and the Szegő condition for orthogonal polynomials on the real line", Comm. Math. Phys., 242:3 (2003), 393-423.

[43] Е.А. Рахманов, "О сходимости диагональных аппроксимаций Паде", Матем. сб., 104(146):2(10) (1977), 271-291; англ. пер.: E. A. Rahmanov, "Convergence of diagonal Padé approximants", Math. USSR-Sb., 33:2 (1977), 243-260.

[44] С.Н. Бернштейн, O многочленах, ортогональных в конечном интервале, ОНТИ, Харьков, 1937.

[45] Г. Сегё, Ортогоналъные многочлены, Физматгиз, М., 1962, 500 с.; пер. с англ.: G. Szegö, Orthogonal polynomials, Amer. Math. Soc. Colloq. Publ., 23, Amer. Math. Soc., Providence, RI, 1959, ix+421 pp.

[46] Е.А. Рахманов, "Об асимптотических свойствах многочленов, ортогональных на вещественной оси”, Матем. сб., 119(161):2(10) (1982), 163-203; англ. пер.: E. A. Rakhmanov, "On asymptotic properties of polynomials orthogonal on the real axis", Math. USSR-Sb., 47:1 (1984), 155-193.

[47] А. А. Гончар, Е. А. Рахманов, "Равновесная мера и распределение нулей экстремальных многочленов", Матем. сб., 125(167):1(9) (1984), 117-127; англ. пер.: A. A. Gonchar, E. A. Rakhmanov, "Equilibrium measure and the distribution of zeros of extremal polynomials", Math. USSR-Sb., 53:1 (1986), 119-130.

[48] А. А. Гончар, Е. А. Рахманов, "О задаче равновесия для векторных потенциалов", УМH, 40:4(244) (1985), 155-156; англ. пер.: А. А. Gonchar, E. A. Rakhmanov, "On the equilibrium problem for vector potentials", Russian Math. Surveys, 40:4 (1985), 183-184.

[49] А.А. Гончар, Е.А. Рахманов, "Равновесные распределения и скорость рациональной аппроксимации аналитических функций”, Матем. сб., 134(176):3(11) (1987), 306-352; англ. пер.: А. A. Gonchar, E. A. Rakhmanov, "Equilibrium distributions and degree of rational approximation of analytic functions", Math. USSR Sb., 62:2 (1989), 305-348.

[50] H. N. Mhaskar, E.B. Saff, "Extremal problems for polynomials with exponential weights", Trans. Amer. Math. Soc., 285:1 (1984), 203-234.

[51] E. B. Saff, V. Totik, Logarithmic potentials with external fields, Grundlehren Math. Wiss., 316, Springer-Verlag, Berlin, 1997, xvi+505 pp.

[52] Е.А. Рахманов, "Об асимптотических свойствах многочленов, ортогональных на вещественной оси”, Докл. АН СССР, 261:2 (1981), 282-284; англ. пер.: E. A. Rakhmanov, "On the asymptotic properties of polynomials orthogonal on the real axis", Dokl. Akad. Nauk SSSR, 261:2 (1981), 282-284.

[53] A. Angelesco, "Sur deux extensions des fractions continues algébriques", C. R. Acad. Sci. Paris, 168 (1919), 262-265.

[54] А. И. Аптекарев, "Асимптотика полиномов совместной ортогональности в случае Анджелеско", Матем. сб., 136(178):1(5) (1988), 56-84; англ. пер.: А. І. Aptekarev, "Asymptotics of simultaneously orthogonal polynomials in the Angelesco case", Math. USSR-Sb., 64:1 (1989), 57-84.

[55] H. N. Mhaskar, E. B. Saff, "Weighted polynomials on finite and infinite intervals: a unified approach", Bull. Amer. Math. Soc. (N.S.), 11:2 (1984), 351-354. 
[56] H.N. Mhaskar, E.B. Saff, "Polynomials with Laguerre weights in $L^{p}$ ", Rational approximation and interpolation (Tampa, FL, 1983), Lecture Notes in Math., 1105, Springer, Berlin, 1984, 511-523.

[57] H. N. Mhaskar, E. B. Saff, "Where does the sup norm of a weighted polynomial live? A generalization of incomplete polynomials", Constr. Approx., 1:1 (1985), 71-91.

[58] P. Deift, Orthogonal polynomials and random matrices: a Riemann-Hilbert approach, Courant Lect. Notes Math., 3, New York University, Courant Institute of Mathematical Sciences, New York; Amer. Math. Soc., Providence, RI, 1999, viii+273 pp.

[59] P. Deift, T. Kriecherbauer, K. T.-R. McLaughlin, S. Venakides, X. Zhou, "Uniform asymptotics for orthogonal polynomials", Proceedings of the International Congress of Mathematicians, Vol. III (Berlin, 1998), Doc. Math., 1998, Extra Vol. III, 491-501.

[60] А. И. Аптекарев, В. Г. Лысов, Д. Н. Туляков, “Глобальный режим распределения собственных значений случайных матриц с ангармоническим потенциалом и внешним источником", ТМФ, 159:1 (2009), 34-57; англ. пер.: А. I. Aptekarev, V.G. Lysov, D. N. Tulyakov, "Global eigenvalue distribution regime of random matrices with an anharmonic potential and an external source", Theoret. Math. Phys., 159:1 (2009), 448-468.

[61] А.И. Аптекарев, В.Г. Лысов, “Системы марковских функций, генерируемые графами, и асимптотика их аппроксимаций Эрмита-Паде", Матем. сб., 201:2 (2010), 29-78; англ. пер.: A. I. Aptekarev, V. G. Lysov, "Systems of Markov functions generated by graphs and the asymptotics of their Hermite-Padé approximants", Sb. Math., 201:2 (2010), 183-234.

[62] Л. Эрдёш, "Универсальность случайных матриц Вигнера: обзор последних результатов", УМH, 66:3(399) (2011), 67-198; англ. пер.: L. Erdős, "Universality of Wigner random matrices: a survey of recent results", Russian Math. Surveys, 66:3 (2011), 507-626.

[63] H. Stahl, "Extremal domains associated with an analytic function. I", Complex Variables Theory Appl., 4:4 (1985), 311-324.

[64] H. Stahl, "Extremal domains associated with an analytic function. II", Complex Variables Theory Appl., 4:4 (1985), 325-338.

[65] H. Stahl, "Structure of extremal domains associated with an analytic function", Complex Variables Theory Appl., 4:4 (1985), 339-354.

[66] H. Stahl, "Orthogonal polynomials with complex valued weight function. I", Constr. Approx., 2:3 (1986), 225-240.

[67] H. Stahl, "Orthogonal polynomials with complex valued weight function. II", Constr. Approx., 2:3 (1986), 241-251.

[68] Ch. Pommerenke, "Padé approximants and convergence in capacity", J. Math. Anal. Appl., 41:3 (1973), 775-780.

[69] H. Stahl, "Three different approaches to convergence problem of diagonal Padé approximants", Rational approximation and applications in mathematics and physics (Łańcut, 1985), Lecture Notes in Math., 1237, Springer, Berlin, 1987, 79-124.

[70] H. Stahl, "The convergence of Padé approximants to functions with branch points", J. Approx. Theory, 91:2 (1997), 139-204.

[71] А.А. Гончар, "Рациональная аппроксимация аналитических функций", Proceedings of the International Congress of Mathematicians, Vol. 1 (Berkeley, CA, 1986), Amer. Math. Soc., Providence, RI, 1987, 739-748; англ. пер.: А. A. Gonchar, "Rational approximations of analytic functions", Nine papers from the International Congress of Mathematicians 1986, Amer. Math. Soc. Transl. Ser. 2, 147, Amer. Math. Soc., Providence, RI, 1990, 25-34.

[72] Р. Варга, Функииональный анализ и теория аппроксимации в численном анализе, Мир, М., 1974, 126 с.; пер. с англ.: R.S. Varga, Functional analysis and 
approximation theory in numerical analysis, CBMS-NSF Regional Conf. Ser. Appl. Math., 3, Society for Industrial and Applied Mathematics, Philadelphia, PA, 1971, $\mathrm{v}+76 \mathrm{pp}$.

[73] M. J. Cody, G. Meinardus, R.S. Varga, "Chebyshev rational approximation to $e^{-x}$ on $[0,+\infty)$ and applications to heat-conduction problems", J. Appr. Theory, 2:1 (1969), 50-65.

[74] E. B. Saff, R.S. Varga, "Some open questions concerning polynomial and rational functions", Padé and rational approximation, Internat. Sympos. (University of South Florida, Tampa, FL, 1976), Academic Press, New York, 1977, 483-488.

[75] A. J. Carpenter, A. Ruttan, R. S. Varga, "Extended computations on the '1/9' conjecture in rational approximation theory", Rational approximation and interpolation (Tampa, FL, 1983), Lecture Notes in Math., 1105, 1984, 383-411.

[76] R. S. Varga, "Scientific computation on some mathematical conjectures", Approximation theory, v. V (College Station, TX, 1986), eds. C. K. Chui, L. L. Schumaker, J. D. Ward, Academic Press, Boston, MA, 1986, 191-209.

[77] G.H. Halphen, Traité des fonctions elliptiques et de leurs applications. Première partie. Théorie des fonctions elliptiques et de leurs développements en séries, Gauthier-Villars, Paris, 1886, viii+492 pp.

[78] A. Martínez-Finkelshtein, "Trajectories of quadratic differentials and approximations of exponents on the semiaxis", Complex methods in approximation theory (Almería, 1995), Monogr. Cienc. Tecnol., 2, Univ. Almeria, Almeria, 1997, 69-84.

[79] A. Martínez-Finkelshtein, R. Orive, "Riemann-Hilbert analysis of Jacobi polynomials orthogonal on a single contour", J. Approx. Theory, 134:2 (2005), 137-170.

[80] S. Kamvissis, E. A. Rakhmanov, "Existence and regularity for an energy maximization problem in two dimensions", J. Math. Phys., 46:8 (2005), 083505, 24 pp.

[81] A. Deano, D. Huybrechs, A. B. J. Kuijlaars, "Asymptotic zero distribution of complex orthogonal polynomials associated with Gaussian quadrature", J. Approx. Theory, 162:12 (2010), 2202-2224; arXiv: 1001.2219.

[82] А.И. Аптекарев, Р.Ф. Хабибуллин, "Асимптотические ряды для многочленов, ортогональных относительно комплексного переменного веса", Тр. ММО, 68, 2006, 3-43; англ. пер.: A. Aptekarev, R. Khabibullin, "Asymptotic expansions for polynomials orthogonal with respect to a complex non-constant weight function", Trans. Moscow Math. Soc., 2007 (2007), 1-37.

[83] А.И. Аптекарев, "Точные константы рациональных аппроксимаций аналитических функций", Матем. сб., 193:1 (2002), 3-72; англ. пер.: А. I. Aptekarev, "Sharp constants for rational approximations of analytic functions", Sb. Math., 193:1 (2002), 1-72.

[84] A.P. Magnus, "On the use of Carathéodory-Fejer method for investigating ' $1 / 9$ ' and similar constants", Nonlinear numerical methods and rational approximation (Wilrijk, 1987), Math. Appl., 43, ed. A. Cuyt, Reidel, Dordrecht, 1988, 105-132.

[85] A. P. Magnus, CFGT determination of Varga's constant '1/9', Preprint B-1348, Institut Mathématique, Université Catholique de Louvain, Belgium, 1986.

[86] A. P. Magnus, "Asymptotics and super asymptotics for best rational approximation error norms to the exponential function (the ' $1 / 9$ ' problem) by the CarathéodoryFejér method", Nonlinear numerical methods and rational approximation, II (Wilrijk, 1993), Math. Appl., 296, eds. A. Cuyt, Kluwer Acad. Publ., Dordrecht, 1994, 173-185.

[87] A. P. Magnus, L. Meinguet, "The elliptic functions and integrals of the ' $1 / 9$ ' problem", Computational methods from rational approximation theory (Wilrijk, 1999), Numer. Algorithms, 24:1-2 (2000), 117-139.

[88] G.A. Baker, Jr., "The existence and convergence of subsequences of Padé approximants", J. Math. Anal. Appl., 43:2 (1973), 498-528. 
[89] G.A. Baker, Jr., Essentials of Padé approximants, Academic Press, New YorkSan Francisco-London, 1975, xi+306 pp.

[90] В. И. Буслаев, А. А. Гончар, С. П. Суетин, "О сходимости подпоследовательностей $m$-й строки таблицы Паде”, Матем. сб., 120(162):4 (1983), 540-545; англ. пер.: V. I. Buslaev, A. A. Gonchar, S. P. Suetin, "On convergence of subsequences of the $m$ th row of a Padé table", Math. USSR-Sb., 48:2 (1984), 535-540.

[91] В. И. Буслаев, "О полюсах $m$-й строки таблицы Паде”, Матем. сб., 117(159):4 (1982), 435-441; англ. пер.: V. I. Buslaev, "On the poles of the $m$ th row of the Padé table", Math. USSR-Sb., 45:4 (1983), 423-429.

[92] E. Fabry, "Sur les points singuliers d'une fonction donnée par son développement en série et l'impossibilité du prolongement analytique dans des cas très généraux", Ann. Sci. École Norm. Sup. (3), 13 (1896), 367-399; http://www.numdam.org/ item?id=ASENS_1896_3_13__367_0.

[93] Л. Бибербах, Аналитическое продолюение, Наука, М., 1967, 241 с.; пер. с нем.: L. Bieberbach, Analytische Fortsetzung, Springer-Verlag, Berlin-Göttingen-Heidelberg, 1955, ii+168 pp.

[94] С. П. Суетин, "О полюсах m-й строки таблицы Паде", Матем. сб., 120(162):4 (1983), 500-504; англ. пер.: S. P. Suetin, "On poles of the $m$ th row of a Padé table", Math. USSR-Sb., 48:2 (1984), 493-497.

[95] С. П. Суетин, "Об одной обратной задаче для $m$-й строки таблицы Паде”, $M a$ тем. сб., 124(166):2(6) (1984), 238-250; англ. пер.: S. P. Suetin, "On an inverse problem for the $m$ th row of the Padé table", Math. USSR-Sb., 52:1 (1985), 231-244.

[96] А. А. Гончар, С.П. Суетин, "Об аппроксимациях Паде мероморфных функций марковского типа", Совр. пробл. матем., 5, МИАН, М., 2004, 3-67; англ. пер.: A. A. Gonchar, S. P. Suetin, "On Padé approximants of Markov-type meromorphic functions", Proc. Steklov Inst. Math., 272, suppl. 2 (2011), 58-95.

[97] С. П. Суетин, "О сильной асимптотике многочленов, ортогональных относительно комплексного веса", Матем. сб., 200:1 (2009), 81-96; англ. пер.: S. P. Suetin, "Strong asymptotics of polynomials orthogonal with respect to a complex weight", Sb. Math., 200:1 (2009), 77-93.

[98] С. П. Суетин, "О равномерной сходимости диагональных аппроксимаций Паде для гиперэллиптических функций”, Матем. сб., 191:9 (2000), 81-114; англ. пер.: S. P. Suetin, "Uniform convergence of Padé diagonal approximants for hyperelliptic functions", Sb. Math., 191:9 (2000), 1339-1373.

[99] С. П. Суетин, "Об асимптотических свойствах полюсов диагональных аппроксимаций Паде для некоторых обобщений марковских функций", Матем. сб., 193:12 (2002), 105-133; англ. пер.: S.P. Suetin, "Approximation properties of the poles of diagonal Padé approximants for certain generalizations of Markov functions", Sb. Math., 193:12 (2002), 1837-1866.

[100] F. Peherstorfer, "Zeros of polynomials orthogonal on several intervals", Int. Math. Res. Not., 2003, № 7, 361-385.

[101] S. Dumas, Sur le développement des fonctions elliptiques en fractions continues, Thèse, Zürich, 1908, 59 pp.

[102] С.П. Суетин, "О сходимости чебышёвских непрерывных дробей для эллиптических функций”, Матем. сб., 194:12 (2003), 63-92; англ. пер.: S. P. Suetin, "Convergence of Chebyshëv continued fractions for elliptic functions", Sb. Math., 194:12 (2003), 1807-1835.

[103] Дж. Спрингер, Введение в теорию римановых поверхностей, ИЛ, М., 1960, 344 с.; пер. с англ.: G. Springer, Introduction to Riemann surfaces, Addison-Wesley, Reading, MA, 1957, viii+307 pp.

[104] H. Stahl, "Convergence of rational interpolants", Bull. Belg. Math. Soc. Simon Stevin, 1996, suppl. issue - Numerical analysis. Papers from the Conference on 
Special Topics in Numerical Analysis and Applied Mathematics in honor of Jean Meinguet (Louvain-la-Neuve, 1995), 11-32.

[105] V.I. Buslaev, "Simple counterexample to the Baker-Gammel-Wills conjecture", East J. Approx., 7:4 (2001), 515-517.

[106] В. И. Буслаев, "О гипотезе Бейкера-Гаммеля-Уиллса в теории аппроксимаций Паде", Матем. сб., 193:6 (2002), 25-38; англ. пер.: V. I. Buslaev, "On the BakerGammel-Wills conjecture in the theory of Padé approximants", Sb. Math., 193:6 (2002), 811-823.

[107] D. S. Lubinsky, "Rogers-Ramanujan and the Baker-Gammel-Wills (Padé) conjecture", Ann. of Math. (2), 157:3 (2003), 847-889.

[108] С.П. Суетин, "Об интерполяционных свойствах диагональных аппроксимаций Паде эллиптических функций”, УМH, 59:4 (2004), 201-202; англ. пер.: S. P. Suetin, "On interpolation properties of diagonal Padé approximants of elliptic functions", Russian Math. Surveys, 59:4 (2004), 800-802.

[109] B. Beckermann, V. Kaliaguine, "The diagonal of the Padé table and the approximation of the Weyl function of second-order difference operators", Constr. Approx., 13:4 (1997), 481-510.

[110] Д.В. Христофоров, "О сходимости диагональных аппроксимаций Паде для эллиптических функций", Матем. сб., 200:6 (2009), 143-160; англ. пер.: D. V. Khristoforov, "On uniform approximation of elliptic functions by Padé approximants", Sb. Math., 200:6 (2009), 923-941.

[111] G. V. Chudnovsky, "On the method of Thue-Siegel", Ann. of Math. (2), 117:2 (1983), 325-382.

[112] G. V. Chudnovsky, "The Thue-Siegel-Roth theorem for values of algebraic functions", Proc. Japan Acad. Ser. A Math. Sci., 59:6 (1983), 281-284.

[113] C. M. Andersen, J.F. Geer, "Power series expansions for the frequency and period of the limit cycle of the van der Pol equation", SIAM J. Appl. Math., 42:3 (1982), 678-693.

[114] M. B. Dadfar, J. Geer, C. M. Andersen, "Perturbation analysis of the limit cycle of the free van der Pol equation", SIAM J. Appl. Math., 44:5 (1984), 881-895.

[115] С. П. Суетин, "Численный анализ некоторых характеристик предельного цикла свободного уравнения Ван дер Поля", Совр. пробл. матем., 14, МИАН, М., 2010, $3-57$.

[116] В.Н. Сорокин, "Аппроксимации Эрмита-Паде для систем Никишина и иррациональность $\zeta(3)$ ", УМH, 49:2(296) (1994), 167-168; англ. пер.: V. N. Sorokin, "Hermite-Padé approximations for Nikishin systems and the irrationality of $\zeta(3)$ ", Russian Math. Surveys, 49:2 (1994), 176-177.

[117] G.L. Litvinov, "Error autocorrection in rational approximation and interval estimates. A survey of results", Cent. Eur. J. Math., 1:1 (2003), 36-60.

[118] С.Б. Гашков, И.Б. Гашков, "Алгоритм Берлекемпа-Месси, цепные дроби, аппроксимации Паде и ортогональные многочлены”, Матем. заметки, 79:1 (2006), 45-59; англ. пер.: S. В. Gashkov, I. B. Gashkov, "Berlekamp-Massey algorithm, continued fractions, Padé approximations, and orthogonal polynomials", Math. Notes, 79:1 (2006), 41-54.

[119] В. Ю. Новокшенов, "Аппроксимации Паде для трансцендентов Пенлеве I и II", TMФ, 159:3 (2009), 515-526; англ. пер.: V. Yu. Novokshenov, "Padé approximations for Painlevé I and II transcendents", Theoret. Math. Phys., 159:3 (2009), 853-862.

[120] А.И. Аптекарев, Е.М. Никишин, "Задача рассеяния для дискретного оператора Штурма-Лиувилля", Матем. сб., 121(163):3(7) (1983), 327-358; англ. пер.: A.I. Aptekarev, E. M. Nikishin, "The scattering problem for a discrete SturmLiouville operator", Math. USSR-Sb., 49:2 (1984), 325-355. 
[121] А.И. Аптекарев, "Асимптотические свойства многочленов, ортогональных на системе контуров, и периодические движения цепочек Тода", Матем. сб., 125(167):2(10) (1984), 231-258; англ. пер.: A. I. Aptekarev, "Asymptotic properties of polynomials orthogonal on a system of contours, and periodic motions of Toda lattices", Math. USSR-Sb., 53:1 (1986), 233-260.

[122] А.И. Аптекарев, "Сильная асимптотика многочленов совместной ортогональности для систем Никишина”, Матем. сб., 190:5 (1999), 3-44; англ. пер.: A. I. Aptekarev, "Strong asymptotics of multiply orthogonal polynomials for Nikishin systems", Sb. Math., 190:5 (1999), 631-669.

[123] В. А. Калягин, "Об одном классе полиномов, определяемых двумя соотношениями ортогональности", Матем. сб., 110(152):4(12) (1979), 609-627; англ. пер.: V. A. Kalyagin, "On a class of polynomials defined by two orthogonality relations", Math. USSR-Sb., 38:4 (1981), 563-580.

[124] В.А. Калягин, "Аппроксимации Эрмита-Паде и спектральный анализ несимметричных операторов", Матем. сб., 185:6 (1994), 79-100; англ. пер.: V. А. Каlyagin, "Hermite-Padé approximants and spectral analysis of nonsymmetric operators", Russian Acad. Sci. Sb. Math., 82:1 (1995), 199-216.

[125] В.А. Калягин, А.А. Кононова, "О компактных возмущениях предельно-периодического оператора Якоби", Матем. заметки, 86:6 (2009), 845-858; англ. пер.: V. A. Kalyagin, A. A. Kononova, "On compact perturbations of the limit-periodic Jacobi operator", Math. Notes, 86:6 (2009), 789-800.

[126] Д. Н. Туляков, "Разностные уравнения с базисами степенного роста, возмущенные спектральным параметром", Матем. сб., 200:5 (2009), 129-158; англ. пер.: D. N. Tulyakov, "Difference equations having bases with powerlike growth which are perturbed by a spectral parameter", Sb. Math., 200:5 (2009), 753-781.

[127] A. Martínez-Finkelshtein, E. A. Rakhmanov, "On asymptotic behavior of HeineStieltjes and Van Vleck polynomials", Recent trends in orthogonal polynomials and approximation theory, Contemp. Math., 507, Amer. Math. Soc., Providence, RI, 2010, 209-232.

[128] A. Martínez-Finkelshtein, E. A. Rakhmanov, "Critical measures, quadratic differentials, and weak limits of zeros of Stieltjes polynomials", Comm. Math. Phys., 302:1 (2011), 53-111; arXiv: 0902.0193.

[129] А. Мартинес-Финкельштейн, Е.А. Рахманов, С. П. Суетин, "Вариация равновесной меры и $S$-свойство стационарного компакта", УMH, 66:1(397) (2011), 183-184; англ. пер.: A. Martínez-Finkelshtein, E. A. Rakhmanov, S. P. Suetin, "Variation of the equilibrium measure and the $S$-property of a stationary compact set", Russian Math. Surveys, 66:1 (2011), 176-178.

[130] А. Мартинес-Финкельштейн, Е. А. Рахманов, С. П. Суетин, "Вариация равновесной энергии и $S$-свойство стационарных компактов", Матем. сб., 202:12 (2011), 113-136.

[131] L. Pastur, "From random matrices to quasi-periodic Jacobi matrices via orthogonal polynomials", J. Approx. Theory, 139:1-2 (2006), 269-292.

[132] A. A. Gonchar, "Some recent convergence results on diagonal Padé approximants", Approximation theory, v. V, Proceedings of the Fifth International Symposium (College Station, TX, 1986), Academic Press, Boston, MA, 1986, 55-70.

[133] А. А. Гончар, Е. А. Рахманов, С. П. Суетин, "О сходимости аппроксимаций Паде ортогональных разложений", Теория чисел, алгебра, математический анализ u ux приложения, Сборник статей. Посвящается 100-летию со дня рождения Ивана Матвеевича Виноградова, Тр. МИАН, 200, Наука, М., 1991, 136-146; англ. пер.: А. А. Gonchar, E. A. Rakhmanov, S. P. Suetin, "On the convergence of Padé approximation of orthogonal expansions", Proc. Steklov Inst. Math., 200 (1993), 149-159. 
[134] A. A. Gonchar, E. A. Rakhmanov, S. P. Suetin, "On the rate of convergence of Padé approximants of orthogonal expansions", Progress in approximation theory (Tampa, FL, 1990), Springer Ser. Comput. Math., 19, Springer, New York, 1992, 169-190.

[135] A. A. Gonchar, E. B. Saff (eds.), Progress in approximation theory, An international perspective. Proceedings of the International Conference on Approximation Theory held at the University of South Florida (Tampa, FL, March 19-22, 1990), Springer Ser. Comput. Math., 19, Springer, New York, 1992, xviii+451 pp.

[136] А. А. Гончар, Е. А. Рахманов, С. П. Суетин, Сходимость нелинейных аппроксимаций Паде-Чебышёва для многозначных аналитических функиий, вариация равновесной энергии и $S$-свойство стационарных компактов, 2010, 49 с., arXiv: 1012.0170 .

[137] А. А. Гончар, Е. А. Рахманов, В. Н. Сорокин, "Об аппроксимациях Эрмита-Паде для систем функций марковского типа”, Матем. сб., 188:5 (1997), 33-58; англ. пер.: A. A. Gonchar, E. A. Rakhmanov, V. N. Sorokin, "Hermite-Padé approximants for systems of Markov-type functions", Sb. Math., 188:5 (1997), 671-696.

[138] С. П. Суетин, "О существовании нелинейных аппроксимаций Паде-Чебышёва для аналитических функций”, Матем. заметки, 86:2 (2009), 290-303; англ. пер.: S.P. Suetin, "On the existence of nonlinear Padé-Chebyshev approximations for analytic functions", Math. Notes, 86:2 (2009), 264-275.

[139] Ю.А. Лабыч, А.П. Старовойтов, “Тригонометрические аппроксимации Паде функций с регулярно убывающими коэффициентами Фурье”, Матем. сб., 200:7 (2009), 107-130; англ. пер.: Yu. A. Labych, A. P. Starovoitov, "Trigonometric Padé approximants for functions with regularly decreasing Fourier coefficients", Sb. Math., 200:7 (2009), 1051-1074.

[140] Н. У. Аракелян, "Об эффективном аналитическом продолжении степенных рядов", Матем. сб., 124(166):1(5) (1984), 24-44; англ. пер.: N. U. Arakelyan, "On efficient analytic continuation of power series", Math. USSR-Sb., 52:1 (1985), 21-39.

[141] Г. Лопес Лагомасино, "Условия сходимости многоточечных аппроксимаций Паде для функций стильтьесовского типа”, Матем. сб., 107(149):1(9) (1978), 69-83; англ. пер.: G. López Lagomasino, "Conditions for convergence of multipoint Padé approximants for functions of Stieltjes type", Math. USSR-Sb., 35:3 (1979), 363-376.

[142] Г. Л. Лопес, “О сходимости аппроксимаций Паде для мероморфных функций стильтьесовского типа", Матем. сб., 111(153):2 (1980), 308-316; англ. пер.: G.L. Lopes, "On the convergence of the Padé approximants for meromorphic functions of Stieltjes type", Math. USSR-Sb., 39:2 (1981), 281-288.

[143] Г. Л. Лопес, "Об асимптотике отношения ортогональных многочленов и сходимости многоточечных аппроксимаций Паде", Матем. сб., 128(170):2(10) (1985), 216-229; англ. пер.: G. L. Lopes, "On the asymptotics of the ratio of orthogonal polynomials and convergence of multipoint Padé approximants", Math. USSR-Sb., 56:1 (1987), 207-219.

[144] Г. Л. Лопес, "Сходимость аппроксимаций Паде мероморфных функций стильтьесовского типа и сравнительная асимптотика для ортогональных многочленов", Матем. сб., 136(178):2(6) (1988), 206-226; англ. пер.: G.L. Lopes, "Convergence of Padé approximants of Stieltjes type meromorphic functions and comparative asymptotics for orthogonal polynomials", Math. USSR-Sb., 64:1 (1989), $207-227$.

[145] Г. Л. Лопес, "Сравнительная асимптотика для многочленов, ортогональных на вещественной оси", Матем. сб., 137(179):4(12) (1988), 500-525; англ. пер.: G.L. Lopes, "Relative asymptotics for polynomials orthogonal on the real axis", Math. USSR-Sb., 65:2 (1990), 505-529. 
[146] Е. А. Рахманов, "Об асимптотике отношения ортогональных многочленов", $M a$ тем. сб., 103(145):2(6) (1977), 237-252; англ. пер.: Е. A. Rahmanov, "On the asymptotics of the ratio of orthogonal polynomials", Math. USSR-Sb., 32:2 (1977), 199-213.

[147] Е.А. Рахманов, "Об асимптотике отношения ортогональных многочленов. II", Матем. сб., 118(160):1(5) (1982), 104-117; англ. пер.: E. A. Rakhmanov, "On the asymptotics of the ratio of orthogonal polynomials. II", Math. USSR-Sb., 46:1 (1983), 105-117.

[148] H. Widom, "Extremal polynomials associated with a system of curves in the complex plane", Adv. Math., 3:2 (1969), 127-232.

[149] J. Nuttall, "Padé polynomial asymptotics from a singular integral equation", Constr. Approx., 6:2 (1990), 157-166.

[150] Э. И. Зверович, "Краевые задачи теории аналитических функций в гёльдеровских классах на римановых поверхностях", УМН, 26:1(157) (1971), 113-179; англ. пер.: E.I. Zverovich, "Boundary value problems in the theory of analytic functions in Hölder classes on Riemann surfaces", Russian Math. Surveys, 26:1 (1971), 117-192.

[151] Б.А. Дубровин, “Тэта-функции и нелинейные уравнения”, УМH, 36:2(218) (1981), 11-80; англ. пер.: В. A. Dubrovin, "Theta functions and non-linear equations", Russian Math. Surveys, 36:2 (1981), 11-92.

[152] С. П. Суетин, "Аппроксимации Паде и эффективное аналитическое продолжение степенного ряда", УМH, 57:1(343) (2002), 45-142; англ. пер.: S. P. Suetin, "Padé approximants and efficient analytic continuation of a power series", Russian Math. Surveys, 57:1 (2002), 43-141.

[153] С. П. Суетин, “Сравнительная асимптотика решений и формулы следов для некоторого класса разностных уравнений", Совр. пробл. матем., 6, МИАН, М., 2006, 3-74; англ. пер.: S.P. Suetin, "Comparative asymptotics of solutions and trace formulas for a class of difference equations", Proc. Steklov Inst. Math., 272, suppl. 2 (2011), 96-137.

[154] С. П. Суетин, "О формулах следов для некоторого класса операторов Якоби", Матем. сб., 198:6 (2007), 107-138; англ. пер.: S. P. Suetin, "Trace formulae for a class of Jacobi operators", Sb. Math., 198:6 (2007), 857-885.

[155] Н.И. Ахиезер, "Об ортогональных многочленах на нескольких интервалах", Докл. АН СССР, 134:1 (1960), 9-12; англ. пер.: N. I. Akhiezer, "Orthogonal polynomials on several intervals", Soviet Math. Dokl., 1 (1960), 989-992.

[156] Н.И. Ахиезер, Ю.Я. Томчук, "К теории ортогональных многочленов на нескольких интервалах", Докл. АН СССР, 138:4 (1961), 743-746; англ. пер.: N.I. Akhiezer, Yu. Ya. Tomchuk, "On the theory of orthogonal polynomials over several intervals", Soviet Math. Dokl., 2 (1961), 687-690.

[157] С. П. Суетин, "Асимптотика полиномов Ахиезера и равномерная сходимость аппроксимаций Паде для гиперэллиптических функций”, УМH, 53:6(324) (1998), 267-268; англ. пер.: S.P. Suetin, "Asymptotics of Akhiezer polynomials and uniform convergence of Padé approximants for hyperelliptic functions", Russian Math. Surveys, 53:6 (1998), 1377-1379.

[158] Н. И. Ахиезер, "Континуальные аналоги ортогональных многочленов на системе интервалов", Докл. АН СССР, 141:2 (1961), 263-266; англ. пер.: N. I. Akhiezer, "A continuous analogue of orthogonal polynomials on a system of intervals", Soviet Math. Dokl., 2 (1961), 1409-1412.

[159] Г.В. Кузьмина, "Модули семейств кривых и квадратичные дифференциалы", Тр. МИАН CССР, 139, 1980, 3-241; англ. пер.: G. V. Kuz'mina, "Moduli of families of curves and quadratic differentials", Proc. Steklov Inst. Math., 139 (1982), $1-231$. 
[160] Г.М. Голузин, Геометрическая теория функиий комплексного переменного, Наука, М., 1966, 628 с.; англ. пер.: G. M. Goluzin, Geometric theory of functions of a complex variable, Transl. Math. Monogr., 26, Amer. Math. Soc., Providence, RI, 1969, vi+676 pp.

[161] В.Н.Дубинин, "Симметризация в геометрической теории функций комплексного переменного", УМH, 49:1(295) (1994), 3-76; англ. пер.: V. N. Dubinin, "Symmetrization in the geometric theory of functions of a complex variable", Russian Math. Surveys, 49:1 (1994), 1-79.

[162] В.Н. Дубинин, Емкости конденсаторов и симметризация в геометрической теории функиий комплексного переменного, Дальнаука, Владивосток, 2009, $390 \mathrm{c}$.

[163] Е. А. Перевозникова, Е. А. Рахманов, Вариация равновесной энергии и S-свойство компактов минимальной емкости, Препринт, М., 1994.

[164] М.А. Лапик, "О носителе экстремальной меры в векторной задаче равновесия", Матем. сб., 197:8 (2006), 101-118; англ. пер.: М. A. Lapik, "Support of the extremal measure in a vector equilibrium problem", Sb. Math., 197:8 (2006), $1205-1221$.

[165] Н.С. Ландкоф, Основы современной теории потенциала, Наука, М., 1966, 515 с.; англ. пер.: N.S. Landkof, Foundations of modern potential theory, Grundlehren Math. Wiss., 180, Springer-Verlag, Berlin-Heidelberg-New York, 1972, $424 \mathrm{pp}$.

[166] В. С. Буяров, Е. А. Рахманов, “О семействах мер, равновесных во внешнем поле на вещественной оси", Матем. сб., 190:6 (1999), 11-22; англ. пер.: V. S. Buyarov, E. A. Rakhmanov, "Families of equilibrium measures in an external field on the real axis", Sb. Math., 190:6 (1999), 791-802.

[167] A. Edrei, "The Padé tables of entire functions", J. Appr. Theory, 28:1 (1980), 54-82.

[168] H. Wallin, "The convergence of Padé approximants and the size of the power series coefficients", Applicable Anal., 4:3 (1974), 235-251.

[169] G. A. Baker, Jr., J. L. Gammel, J. G. Wills, "An investigation of the applicability of the Padé approximant method", J. Math. Anal. Appl., 2:3 (1961), 405-418.

[170] В.И. Буслаев, "Соотношения для коэффициентов и особые точки функции", Матем. сб., 131(173):3(11) (1986), 357-384; англ. пер.: V. I. Buslaev, "Relations for the coefficients, and singular points of a function", Math. USSR-Sb., 59:2 (1988), 349-377.

[171] В. И. Буслаев, "Аналог теоремы Фабри для обобщенных аппроксимаций Паде", Матем. сб., 200:7 (2009), 39-106; англ. пер.: V. I. Buslaev, "An analogue of Fabry's theorem for generalized Padé approximants", Sb. Math., 200:7 (2009), 981-1050.

[172] S. Uchiyama, "Rational approximations to algebraic functions", J. Fac. Sci. Hokkaido Univ. Ser. I, 15 (1961), 173-192.

[173] H. Stahl, "A note on three conjectures by Gonchar on rational approximation", J. Approx. Theory, 50:1 (1987), 3-7.

[174] H. Stahl, "Conjectures around the Baker-Gammel-Wills conjecture", Constr. Approx., 13:2 (1997), 287-292.

[175] G. Pólya, "Untersuchungen über Lücken und Singularitäten von Potenzreihen", Math. Z., 29:1 (1929), 549-640.

[176] Дж.Л. Уолш, Интерполяция и аппроксимачия рациональными функииями в комплексной плоскости, ИЛ, М., 1961, 508 с.; пер. с англ.: J.L. Walsh, Interpolation and approximation by rational functions in the complex domain, Amer. Math. Soc. Colloq. Publ., XX, Amer. Math. Soc., Providence, RI, 1960, x+398 pp.

[177] О.Г. Парфенов, "Оценки сингулярных чисел оператора вложения Карлесона", Матем. сб., 131(173):4(12) (1986), 501-518; англ. пер.: О. G. Parfenov, 
"Estimates of the singular numbers of the Carleson imbedding operator", Math. USSR-Sb., 59:2 (1988), 497-514.

[178] В. А. Прохоров, “Об одной теореме Адамяна-Арова-Крейна”, Матем. сб., 184:1 (1993), 89-104; англ. пер.: V. A. Prokhorov, "On a theorem of Adamian, Arov, and Krein", Russian Acad. Sci. Sb. Math., 78:1 (1994), 77-90.

[179] В.А. Прохоров, "Рациональная аппроксимация аналитических функций", Maтем. сб., 184:2 (1993), 3-32; англ. пер.: V. A. Prokhorov, "Rational approximation of analytic functions", Russian Acad. Sci. Sb. Math., 78:1 (1994), 139-164.

[180] В. А. Прохоров, "О скорости рациональной аппроксимации мероморфных функций”, Матем. сб., 185:1 (1994), 3-26; англ. пер.: V. A. Prokhorov, "On the degree of rational approximation of meromorphic functions", Russian Acad. Sci. Sb. Math., 81:1 (1995), 1-20.

[181] H. Stahl, "Diagonal Padé approximants to hyperelliptic functions", Ann. Fac. Sci. Toulouse Math. (6), 1996, Special Issue, 121-193.

[182] А. А. Гончар, "О задачах Е. И. Золотарева, связанных с рациональными функциями", Матем. сб., 78(120):4 (1969), 640-654; англ. пер.: А. A. Gonchar, "Zolotarev problems connected with rational functions", Math. USSR-Sb., 7:4 (1969), 623-635.

[183] H. Poincaré, "Sur les équations linéaires aux différentielles ordinaires et aux différences finies", Amer. J. Math., 7:3 (1885), 203-258.

[184] O. Perron, "Über einen Satz des Herrn Poincaré", J. Reine Angew. Math., 136 (1909), 17-37.

[185] O. Perron, "Über die Poincarésche lineare Differenzengleichung", J. Reine Angew. Math., 137 (1910), 6-64.

[186] М. А. Евграфов, "Новое доказательство теоремы Перрона", Изв. АН СССР. Сер. матем., 17:2 (1953), 77-82.

[187] А. О. Гельфонд, Исчисление конечных разностей, Наука, М., 1967, 375 с.; англ. пер.: A. O. Gel'fond, Calculus of finite differences, Int. Monogr. Advanced Math. Phys., Hindustan Publishing Corp., Delhi, 1971, vi+451 pp.

[188] Г.А. Фрейман, “О теоремах Пуанкаре и Перрона”, УМH, 12:3(75) (1957), 241-246.

[189] В.И. Буслаев, "О теореме Пуанкаре и ее приложениях к вопросам сходимости цепных дробей”, Матем. сб., 189:12 (1998), 13-28; англ. пер.: V.I. Buslaev, "Poincaré's theorem and its applications to the convergence of continued fractions", Sb. Math., 189:12 (1998), 1749-1764.

[190] E. B. Van Vleck, "On the convergence of algebraic continued fractions whose coefficients have limiting values", Trans. Amer. Math. Soc., 5:3 (1904), 253-262.

[191] В.И. Буслаев, "О теореме Ван Флека для правильных $C$-дробей с предельно периодическими коэффициентами", Изв. РАН. Сер. матем., 65:4 (2001), 35-48; англ. пер.: V.I. Buslaev, "On the Van Vleck theorem for regular $C$-fractions with limit-periodic coefficients", Izv. Math., 65:4 (2001), 673-686.

[192] В.И. Буслаев, "О сходимости непрерывных Т-дробей”, Аналитические и геометрические вопросы комплексного анализа, Сборник статей. K 70-летию со дня рождения академика Анатолия Георгиевича Витушкина, Тр. МИАН, 235, Наука, M., 2001, 36-51; англ. пер.: V. I. Buslaev, "On the convergence of continued T-fractions", Proc. Steklov Inst. Math., 235 (2001), 29-43.

[193] G. Pólya, "Über gewisse notwendige Determinantkriterien für die Forsetzbarkeit einer Potenzreihe", Math. Ann., 99:1 (1928), 687-706.

[194] В.И. Буслаев, "Оценка емкости множества особенностей функций, заданных своим разложением в непрерывную дробь”, Anal. Math., 2012 (в печати). 
[195] J. Worpitsky, "Untersuchungen über die Entwickelung der monodromen und monogenen Funktionen durch Kettenbrüche", Friedrichs-Gymnasium und Realschule Jahresbericht, Berlin, 1865, 3-39.

[196] W. T. Scott, H. S. Wall, "Continued fraction expansions for arbitrary power series", Ann. Math. (2), 41 (1940), 328-349.

[197] А. А. Гончар, "Об особых точках мероморфных функций, заданных своим разложением в С-дробь", Матем. сб., 197:10 (2006), 3-14; англ. пер.: А. A. Gonchar, "Singular points of meromorphic functions defined by their expansion in a C-fraction", Sb. Math., 197:10 (2006), 1405-1416.

[198] M. D. Hirschhorn, "Partitions and Ramanujan's continued fraction", Duke Math. J., 39 (1972), 789-791.

[199] G.H. Hardy, J.E. Littlewood, "Notes on the theory of series. XXIV. A curious power-series", Proc. Cambridge Philos. Soc., 42 (1946), 85-90.

[200] D. S. Lubinsky, "Note on polynomial approximation of monomials and Diophantine approximation", J. Approx. Theory, 43:1 (1985), 29-35.

[201] G. Petruska, "On the radius of convergence of $q$-series", Indag. Math. (N.S.), 3:3 (1992), 353-364.

[202] В. И. Буслаев, "О сходимости непрерывной дроби Роджерса-Рамануджана", Maтем. сб., 194:6 (2003), 43-66; англ. пер.: V. I. Buslaev, "Convergence of the Rogers-Ramanujan continued fraction", Sb. Math., 194:6 (2003), 833-856.

[203] В. И. Буслаев, “О теореме Фабри об отношении для ортогональных рядов”, Комплексный анализ и приложения, Сборник статей, Тр. МИАН, 253, Наука, М., 2006, 14-29; англ. пер.: V. I. Buslaev, "On the Fabry ratio theorem for orthogonal series", Proc. Steklov Inst. Math., 253 (2006), 8-21.

[204] С. П. Суетин, "Обратные теоремы об обобщенных аппроксимациях Паде”, $M a$ тем. сб., 109(151):4(8) (1979), 629-646; англ. пер.: S. P. Suetin, "Inverse theorems on generalized Padé approximants", Math. USSR-Sb., 37:4 (1980), 581-597.

\section{А. И. Аптекарев (А. I. Aptekarev)}

Институт прикладной математики

им. М. В. Келдыша РАН

E-mail: aptekaa@keldysh.ru

\section{В. И. Буслаев (V. I. Buslaev)}

Математический институт им. В. А. Стеклова РАН

E-mail: buslaev@mi.ras.ru

\section{А. Мартинес-Финкельштейн}

(A. Martínez-Finkelshtein)

University of Almeria, Spain

E-mail: andrei@ual.es

\section{С. П. Суетин (S. P. Suetin)}

Математический институт им. В. А. Стеклова РАН

E-mail: suetin@mi.ras.ru
Поступила в редакцию 03.10 .2011 